TRANSACTIONS OF THE

AMERICAN MATHEMATICAL SOCIETY

Volume 365, Number 11, November 2013, Pages 5605-5654

S 0002-9947(2013)05998-3

Article electronically published on June 28, 2013

\title{
SHORT-TIME EXISTENCE OF THE RICCI FLOW ON NONCOMPACT RIEMANNIAN MANIFOLDS
}

\author{
GUOYI XU
}

\begin{abstract}
In this paper, we give the first detailed proof of the short-time existence of Deane Yang's local Ricci flow. Then, using the local Ricci flow, we prove the short-time existence of the Ricci flow on noncompact manifolds, whose Ricci curvature has global lower bound and sectional curvature has only local average integral bound. The short-time existence of the Ricci flow on noncompact manifolds with bounded curvature was studied by Wan-Xiong Shi in the 1990s. As a corollary of our main theorem, we obtain the short-time existence part of Shi's theorem in this more general context.
\end{abstract}

\section{INTRODUCTION}

In his well-known paper 5, R. Hamilton introduced the Ricci flow on compact Riemannian manifolds which has proved to be very useful in the research of differential geometry and topology. Let us recall the definition of the Ricci flow: it is the solution of the evolution equation deforming the metric on any $n$-dimensional Riemannian manifold $\left(M^{n}, g_{i j}\right)$,

$$
\left\{\begin{aligned}
\frac{\partial}{\partial t} g_{i j}(x, t) & =-2 R_{i j}(x, t) \\
g(x, 0) & =g_{0}(x)
\end{aligned}\right.
$$

where $R_{i j}$ is the Ricci curvature of $M^{n}$ at time $t$.

The first thing we mention in the study of the Ricci flow on Riemannian manifolds is the short-time existence of the solution. In the case where $M^{n}$ is compact, it is well known that for any given initial metric $g_{i j}$ on $M^{n}$, the evolution equation (1.1) always has a unique solution for a short time (see [4, 5]).

To prove Yau's Uniformization Conjecture, W.-X. Shi initiated studying the Ricci flow on complete noncompact Riemannian manifolds. The short-time existence problem of the evolution equation (1.1) is more difficult than the compact case. In [12], Shi proved that if $\left(M^{n}, g_{i j}\right)$ is complete noncompact with bounded curvature, then the Ricci flow (1.1) has a solution with bounded curvature on a short-time interval. Note that Shi's theorem requires that the initial manifold $\left(M^{n}, g_{0}\right)$ has the bounded geometry, i.e. the curvature of $M^{n}$ must have a point-wise estimate.

In 15, Deane Yang introduced the local Ricci flow. We recall the definition of local Ricci flow first. Let $M^{n}$ be a smooth $n$-dimensional manifold with Riemannian metric $g_{0}, \Omega$ an open bounded domain of $M^{n}$ and $n \geq 3$. Let $\chi$ be a nonnegative

Received by the editors December 28, 2010.

2010 Mathematics Subject Classification. Primary 35K45, 53C44.

(C) 2013 American Mathematical Society Reverts to public domain 28 years from publication 
smooth compactly supported function on $\Omega$, and $0 \leq \chi \leq 1$. The local Ricci flow is the solution of the following evolution equation:

$$
\frac{\partial g}{\partial t}=-2 \chi^{2} R c(g), \quad g(0)=g_{0}, \quad \chi \in C_{0}^{\infty}(\Omega) .
$$

In this paper, using the local Ricci flow, we prove

Theorem 1.1. Assume $\left(M^{n}, g_{0}\right)$ is an $n$-dimensional $(n \geq 3)$ complete noncompact Riemannian manifold, satisfying the following conditions:

$$
\left\{\begin{aligned}
\left(f_{B_{x}\left(4 r_{0}\right)} h^{\frac{2 n}{n-2}} d V_{g_{0}}\right)^{\frac{n-2}{n}} & \leq A_{0} r_{0}^{2} f_{B_{x}\left(4 r_{0}\right)}|\nabla h|^{2} d V_{g_{0}}, \\
\left(f_{B_{x}\left(r_{0}\right)}\left|R m\left(g_{0}\right)\right|^{p_{0}} d V_{g_{0}}\right)^{\frac{1}{p_{0}}} & \leq K_{1}
\end{aligned}\right.
$$

for any $x \in M$ and $h \in C_{0}^{\infty}\left(B_{x}\left(4 r_{0}\right)\right)$, where $A_{0} \geq 1, p_{0}>\frac{n}{2}, K_{1}$ and $r_{0}$ are positive constants.

Then the Ricci flow (1.1) has a smooth solution $g_{i j}(x, t)$ on $\left[0, \frac{T_{2}}{2}\right]$ (where $T_{2}$ is defined in (5.25) ) and satisfies the following estimates. For any integer $m \geq 0$, there exists a positive constant $C\left(A_{0}, K_{1}, m, n, p_{0}, r_{0}\right)$ depending only on $A_{0}, K_{1}$, $m, n, p_{0}$ and $r_{0}$, such that

$$
\sup _{x \in M}\left|\nabla^{m} R m(x, t)\right| \leq \frac{C\left(A_{0}, K_{1}, m, n, p_{0}, r_{0}\right)}{t^{\frac{m}{2}}}\left(t^{-\frac{n}{2 p_{0}}}+t^{\frac{1}{p_{0}}}\right), \quad 0<t \leq \frac{T_{2}}{2} .
$$

In particular,

$$
\sup _{x \in M}|R m(x, t)| \leq C_{2}\left(t^{-\frac{n}{2 p_{0}}}+C_{1}^{\frac{n+2}{2 p_{0}}} t^{\frac{1}{p_{0}}}\right), \quad 0<t \leq \frac{T_{2}}{2},
$$

where $C_{1}$ and $C_{2}$ are defined in (5.9) and (5.16).

Theorem 1.1 requires only the integral conditions on the curvature tensor of the initial manifold and a local Sobolev inequality. By [10, local Sobolev inequality is valid on Riemannian manifolds whose Ricci curvature has a lower bound. Hence we can generalize Shi's theorem in more general context in the following corollary.

Corollary 1.2. Assume $\left(M, g_{0}\right)$ is an $n$-dimensional $(n \geq 3)$ complete noncompact Riemannian manifold, satisfying the following conditions:

$$
\left\{\begin{aligned}
R c\left(g_{0}\right) & \geq-K g_{0}, \\
\left(f_{B_{x}\left(r_{0}\right)}\left|R m\left(g_{0}\right)\right|^{p_{0}} d V_{g_{0}}\right)^{\frac{1}{p_{0}}} & \leq K_{1}
\end{aligned}\right.
$$

for any $x \in M$, where $p_{0}>\frac{n}{2}, r_{0}, K$ and $K_{1}$ are positive constants.

Then the Ricci flow (1.1) has a smooth solution $g_{i j}(x, t)$ on $[0, T]$, and satisfies the following estimates. For any integer $m \geq 0$, there exists the positive constant $C\left(K, K_{1}, m, n, p_{0}, r_{0}\right)$ such that

$$
\sup _{x \in M}\left|\nabla^{m} R m(x, t)\right| \leq \frac{C\left(K, K_{1}, m, n, p_{0}, r_{0}\right)}{t^{\frac{m}{2}}}\left(t^{-\frac{n}{2 p_{0}}}+t^{\frac{1}{p_{0}}}\right), \quad 0<t \leq T,
$$

where $T=T\left(K, K_{1}, n, p_{0}, r_{0}\right)$ is a positive constant depending only on $K, K_{1}, n$, $p_{0}$ and $r_{0}$.

In section 2, we give the detailed proof of short-time existence of the local Ricci flow following the brief sketch in [15]. In the sketch proof of Theorem 8.2 of [15], 
Yang considered a similar but different differential system (see the top of page 91 of [15]) comparing (2.4), and the main difference is that our principal coefficients in (2.4) are $\left(\chi^{2}+\epsilon\right) u^{\alpha \beta}$ instead of $\left(\chi^{2}+\epsilon\right) v^{\alpha \beta}$. This is the key point as to why we can get the $C^{0}$ a-priori estimate of $v_{i j}$ (see (2.11), etc.) and the higher order estimate further on in Proposition 2.5.

More concretely, for (2.4) we prove that $\Phi_{\epsilon}: U \rightarrow V$ is a contraction map on $[0, T]$, where $T$ is independent of $\epsilon$. Then we use the fixed point method to get a solution of (2.4) on $[0, T]$. Finally, letting $\epsilon \rightarrow 0$, we get the solution of (2.1) on $[0, T]$, and by regularity of parabolic system, the solution is smooth. All the estimates are about metric tensors, and the norms we considered are with respect to the initial metric $g_{0}$ of manifold $M^{n}$.

In spirit, our proof is close to the original proof of the Ricci flow's short-time existence on compact manifolds by R. Hamilton. In [5], R. Hamilton used the NashMoser inverse function theorem; in this paper we use the fixed-point argument in a Sobolev-type inequality.

In section 3, we discuss the evolution equations and inequalities of curvature tensors under local Ricci flow. In section 4, the extension of local Ricci flow is discussed. The main theorem (Theorem 4.8) of this section was proved in [15]; recently the complete proof was given by Wang, among other things (see [14]). The proof is nontrivial and different from the proof of the Ricci flow's extension under the bound of curvature. In the proof, the interplay among the estimates of $\nabla^{i} \chi$, $\nabla^{j} R m$ and $\nabla^{k}\left(\chi^{2} R m\right)$ are interesting and subtle. Following the proof of Wang, we prove this extension theorem again to make this paper self-contained. Note all the estimates in sections 2, 3 and 4 are valid for any smooth initial manifold $\left(M^{n}, g_{0}\right)$.

After establishing the short-time existence of the local Ricci flow and its extension theorem, we try to get the existence time estimate of the local Ricci flow independent of the domain $\Omega$. This is done in section 5 and section 6 . Note that the time estimate which we get in the proof of the short-time existence of the local Ricci flow depends on $\Omega$. To get the time estimate independent of $\Omega$, we need to add some assumptions on the initial manifold $\left(M^{n}, g_{0}\right)$.

The key point of section 5 is: If we assume the initial local $L^{p}\left(p>\frac{n}{2}\right)$ average integral bound of curvature tensor and local average Sobolev inequality, we can get the parabolic version of the "local local" energy estimate of curvature tensor, where the coefficient of $f \xi_{i}^{2 q-2} \chi^{2 p^{\prime}}|R m|^{p}$ is independent of $\Omega$. Here "local local" means that we use two cut-off functions with curvature tensor. One is $\xi_{i}$, which is from the partition of unity of the good cover mentioned before, and the other is $\chi$, which is the cut-off function in the local Ricci flow.

Then using the modified Moser iteration, we can get the point-wise estimate of $\chi^{2} R m$ on $[0, T]$, where $T$ is independent of $\Omega$. By the local Ricci flow's extension theorem proved in section 4 , we get our existence-time estimate independent of $\Omega$. The precise-time estimate we get is expressed in the explicit form of curvature bound, diameter of balls in good cover and local Sobolev constant, and this explicit estimate has independent interest and application (see Remarks 5.15] and 7.1). Another reason we get a time estimate in such an explicit expression (with $A_{0}, r_{0}, K_{1}$, see (5.21) and (5.24), etc.) is that we are interested in the $R c \geq 0$ case, where we can allow $r_{0} \rightarrow \infty$ if there is a suitable initial curvature integral bound on $B\left(r_{0}\right)$ as $r_{0} \rightarrow \infty$.

In many situations, critical metrics are interesting, and we have only the $L^{\frac{n}{2}}$ bound of $R m$. In section 6 , using different energy estimates we get a similar 
existence-time estimate of the local Ricci flow on manifolds with a small $L^{\frac{n}{2}}$ bound of $R m$, an $L^{p}\left(p>\frac{n}{2}\right)$ bound of $R c$, local Sobolev inequality and volume doubling property.

In section 7 , using a family of local Ricci flows with respect to a family of expanding domains $\left\{\Omega_{i}\right\}_{i=1}^{\infty}$ and the curvature estimates we get in sections 5 and 6 , we can apply compactness results in Ricci flow to show that a subsequence of this family of local Ricci flows converges to the Ricci flow on the whole manifold when $i \rightarrow \infty$.

Finally, it is worth mentioning that our arguments in sections 5 and 6 also apply to compact manifolds. On compact manifolds, we can choose $\chi \equiv 1$ in (1.2); then local Ricci flow is just Ricci flow. Note the minimal existence time dependent on curvature bound is well known (see Corollary 7.7 in [1]), and the minimal existence time we get depends on the integral bound of curvature, which has its own interest.

\section{Short-time existence of the LOCAL Ricci Flow}

To prove the short-time existence of the local Ricci flow, we follow the brief sketch in the paper of Deane Yang [15]. By DeTurck's trick, we can equivalently consider the following local Ricci-DeTurck flow:

$$
\left\{\begin{aligned}
\frac{\partial}{\partial t} g_{i j} & =-2 \chi^{2} R_{i j}+\nabla_{i}\left(\chi^{2} \mathscr{W}\right)_{j}+\nabla_{j}\left(\chi^{2} \mathscr{W}\right)_{i} \\
\frac{\partial}{\partial t} \phi_{t}(p) & =-\chi^{2}(p) \mathscr{W}\left(\phi_{t}(p), t\right), \quad p \in M \\
\phi_{0} & =I d_{M}, \quad g_{i j}(x, 0)=\tilde{g}_{i j}(x) \triangleq\left(g_{0}\right)_{i j}(x)
\end{aligned}\right.
$$

where $\mathscr{W}_{j}=g_{j k} g^{p q}\left(\Gamma_{p q}^{k}-\tilde{\Gamma}_{p q}^{k}\right)$. The second equation in (2.1) is a quasilinear ODE, and the solution on $M \backslash \Omega$ is $I d_{M \backslash \Omega}$, so one only need consider the first family of equations in (2.1).

Lemma 2.1. The following modified evolution equations (2.2) is a degenerate parabolic system:

$$
\left\{\begin{aligned}
\frac{\partial}{\partial t} g_{i j} & =-2 \chi^{2} R_{i j}+\nabla_{i}\left(\chi^{2} \mathscr{W}\right)_{j}+\nabla_{j}\left(\chi^{2} \mathscr{W}\right)_{i}, \\
g_{i j}(x, 0) & =\tilde{g}_{i j}(x) .
\end{aligned}\right.
$$

Proof. On $M \backslash \Omega, g_{i j}(x, t) \equiv \tilde{g}_{i j}(x)$, one only needs to consider the evolution equations on $\Omega$. Calculating (2.2) directly, using Lemma 2.1 in [12, we get

$$
\left\{\begin{aligned}
\frac{\partial}{\partial t} g_{i j}= & \chi^{2} g^{\alpha \beta} \tilde{\nabla}_{\alpha} \tilde{\nabla}_{\beta} g_{i j}-\chi^{2}\left(g^{\alpha \beta} g_{i p} \tilde{g}^{p q} \tilde{R}_{j \alpha q \beta}+g^{\alpha \beta} g_{j p} \tilde{g}^{p q} \tilde{R}_{i \alpha q \beta}\right) \\
& +\frac{1}{2} \chi^{2} g^{\alpha \beta} g^{p q}\left(\tilde{\nabla}_{i} g_{p \alpha} \tilde{\nabla}_{j} g_{q \beta}+2 \tilde{\nabla}_{\alpha} g_{j p} \tilde{\nabla}_{q} g_{i \beta}-2 \tilde{\nabla}_{\alpha} g_{j p} \tilde{\nabla}_{\beta} g_{i q}\right. \\
& \left.-2 \tilde{\nabla}_{j} g_{p \alpha} \tilde{\nabla}_{\beta} g_{i q}-2 \tilde{\nabla}_{i} g_{p \alpha} \tilde{\nabla}_{\beta} g_{j q}\right) \\
& +\chi \chi_{i} g^{p q}\left(\tilde{\nabla}_{p} g_{j q}+\tilde{\nabla}_{q} g_{p j}-\tilde{\nabla}_{j} g_{p q}\right)+\chi \chi_{j} g^{p q}\left(\tilde{\nabla}_{p} g_{i q}+\tilde{\nabla}_{q} g_{p i}-\tilde{\nabla}_{i} g_{p q}\right) \\
g_{i j}(x, 0)= & \tilde{g}_{i j}(x), \quad x \in \Omega, \\
g_{i j}(x, t)= & \tilde{g}_{i j}(x), \quad x \in \partial \Omega, t \in[0, T],
\end{aligned}\right.
$$

where $\chi_{i}=\tilde{\nabla}_{i} \chi$. Note that the principal coefficients of (2.3) are $\left(\chi^{2} g^{\alpha \beta}\right)$, and it is a degenerate parabolic system. 
We define the following tensor-valued function spaces and norms:

\section{Definition 2.2.}

$$
\begin{aligned}
\mathfrak{A}_{T} & =\left\{U \mid \frac{1}{2} \tilde{g}_{i j} \leq u_{i j} \leq 2 \tilde{g}_{i j}, U(x, 0)=\tilde{g}(x), x \in \Omega\right. \\
U(x, t) & \left.=\tilde{g}, x \in \partial \Omega, U \in L^{\infty}\left(\bar{\Omega} \times[0, T], \otimes^{2} T^{*}(\bar{\Omega})\right)\right\} \\
|U(x, t)|_{k} & =\left(\sum_{|\alpha| \leq k} \int_{\Omega}\left|\tilde{\nabla}^{\alpha} U(x, t)\right|^{2} d \mu_{\tilde{g}}\right)^{\frac{1}{2}}, \quad|U(x, t)|_{T, k}=\sup _{t \in[0, T]}\left(|U(x, t)|_{k}\right), \\
\|f\|_{k} & \triangleq \sum_{|\alpha| \leq k}\left|\tilde{\nabla}^{\alpha} f\right|_{L^{\infty}(\Omega)}, \quad k=0,1,2, \cdots .
\end{aligned}
$$

where $U:\left(\bar{\Omega} \times[0, T] \rightarrow \otimes^{2} T^{*}(\bar{\Omega})\right), f$ is a tensor field or function defined on $\Omega$ and $\left|\nabla^{\alpha} U(x, t)\right|^{2}=\tilde{g}^{i_{1} j_{1}} \cdots \tilde{g}^{i_{s} j_{s}} \tilde{g}^{p k} \tilde{g}^{q l}\left(\tilde{\nabla}_{i_{1}} \cdots \tilde{\nabla}_{i_{s}} u_{p q}\right)\left(\tilde{\nabla}_{j_{1}} \cdots \tilde{\nabla}_{j_{s}} u_{k l}\right)$, where $|\alpha|=s \geq$ 0. Also,

$$
\mathbb{B}_{m}(K)_{T}=\left\{\left.U|| U\right|_{T, m} \leq K ; U \in L^{\infty}\left(\bar{\Omega} \times[0, T], \otimes^{2} T^{*}(\bar{\Omega})\right)\right\},
$$

where $m \geq 0, K>0$.

We consider the following strictly parabolic system by adding $\epsilon$ to $\chi^{2}$ in the principal coefficients, and $\epsilon \leq 1$ is some positive constant:

$$
\left\{\begin{aligned}
\frac{\partial}{\partial t} v_{i j} & =\left(\chi^{2}+\epsilon\right) u^{\alpha \beta}\left(v_{i j}\right)_{\alpha \beta}+D_{i j}(U, V)+B_{i j}(U, \tilde{\nabla} U), \\
v_{i j}(x, 0) & =\tilde{g}_{i j}(x), \quad x \in \Omega, \\
v_{i j}(x, t) & =\tilde{g}_{i j}(x), \quad x \in \partial \Omega, t \in[0, T],
\end{aligned}\right.
$$

where $U=\left(u_{i j}\right)_{i, j=1}^{n}, V=\left(v_{i j}\right)_{i, j=1}^{n},\left(v_{i j}\right)_{\alpha \beta}=\widetilde{\nabla}_{\alpha} \widetilde{\nabla}_{\beta}\left(v_{i j}\right)$,

$$
\begin{aligned}
D_{i j}(U, V)= & -\chi^{2}\left[\left(u^{\alpha \beta} \tilde{g}^{p q} \tilde{R}_{j \alpha q \beta}\right) v_{i p}+\left(u^{\alpha \beta} \tilde{g}^{p q} \tilde{R}_{i \alpha q \beta}\right) v_{j p}\right], \\
B_{i j}(U, \tilde{\nabla} U)= & E_{i j}+F_{i j}, \\
E_{i j}= & \frac{1}{2} \chi^{2} u^{\alpha \beta} u^{p q}\left(\tilde{\nabla}_{i} u_{p \alpha} \tilde{\nabla}_{j} u_{q \beta}+2 \tilde{\nabla}_{\alpha} u_{j p} \tilde{\nabla}_{q} u_{i \beta}-2 \tilde{\nabla}_{\alpha} u_{j p} \tilde{\nabla}_{\beta} u_{i q}\right. \\
& \left.-2 \tilde{\nabla}_{j} u_{p \alpha} \tilde{\nabla}_{\beta} u_{i q}-2 \tilde{\nabla}_{i} u_{p \alpha} \tilde{\nabla}_{\beta} u_{j q}\right), \\
F_{i j}= & \chi \chi_{i} u^{p q}\left(\tilde{\nabla}_{p} u_{j q}+\tilde{\nabla}_{q} u_{p j}-\tilde{\nabla}_{j} u_{p q}\right)+\chi \chi_{j} u^{p q}\left(\tilde{\nabla}_{p} u_{i q}+\tilde{\nabla}_{q} u_{p i}-\tilde{\nabla}_{i} u_{p q}\right) .
\end{aligned}
$$
Then

$$
\frac{\partial}{\partial t} v_{i j}=A_{\epsilon}^{\alpha \beta}\left(v_{i j}\right)_{\alpha \beta}+D_{i j}(U, V)+B_{i j}(U, \tilde{\nabla} U) .
$$

Remark 2.3. In (2.5), there is no $\nabla V$ term, and this is one of the reasons we can get a $C^{0}$-estimate of $V$ in Proposition 2.5.

We first prove a technical lemma about the norm of $U^{-1}$ using interpolation inequalities. We define the Sobolev constant $C_{s}(\Omega, g(t))$ for $\Omega \subset(M, g(t))$ as the following:

$$
|h|_{C^{0}(\Omega)} \leq C_{s}(\Omega, g(t))\left(\int_{\Omega}|\nabla h|_{g(t)}^{2} d V_{g(t)}\right)^{\frac{1}{2}}, \quad \forall h \in C_{0}^{\infty}(\Omega) .
$$

In this section we use the notation $C_{s}\left(\Omega, g_{0}\right) \triangleq C_{s}(\Omega, \tilde{g})$ because the covariant derivatives, tensor norm considered in this section are all with respect to $\tilde{g}$. 
Choose $G=2 \sqrt{n} V_{\tilde{g}}(\Omega)^{\frac{1}{2}}$ and some fixed $\tilde{p}>\min \left\{\frac{n}{2}, 3\right\}$ in the rest of this section. Note $|\tilde{g}|_{m}=\sqrt{n} V_{\tilde{g}}(\Omega)^{\frac{1}{2}}$ for any $m \geq 0$.

Lemma 2.4. Assume $U \in \mathfrak{A}_{T}$ and $\left|U^{-1}(x, t)\right|_{L^{\infty}} \leq C_{0}$ for some $t \in[0, T]$. Then

$$
\left|U^{-1}(x, t)\right|_{k+1} \leq C\left(k, C_{0}, C_{s}\left(\Omega, g_{0}\right)\right)\left(|U(x, t)|_{\tilde{p}+1}+1\right)^{k+1}\left(|U(x, t)|_{k+1}+1\right),
$$

where $k=0,1,2, \cdots$.

Proof.

$$
\begin{aligned}
& \left|\tilde{\nabla}^{k+1} U^{-1}\right|_{L^{2}}=\left|\tilde{\nabla}^{k}\left(U^{-1} U^{-1} \tilde{\nabla} U\right)\right|_{L^{2}}=\left|\tilde{\nabla}^{k}\left(U^{-2} \tilde{\nabla} U\right)\right|_{L^{2}} \\
& \leq C(k)\left(\left(\left|U^{-2}\right|_{L^{\infty}}+1\right)|\tilde{\nabla} U|_{k}+\left|U^{-2}\right|_{k}\left(|\tilde{\nabla} U|_{L^{\infty}}+1\right)\right) \\
& \leq C\left(k, C_{0}\right)|\tilde{\nabla} U|_{k}+C\left(k, C_{0}, C_{s}\left(\Omega, g_{0}\right)\right)\left|U^{-1}\right|_{k}\left(\left|U^{-1}\right|_{L^{\infty}}+1\right)\left(|U|_{\tilde{p}+1}+1\right) \\
& \leq C\left(k, C_{0}, C_{s}\left(\Omega, g_{0}\right)\right)|U|_{k+1}+C\left(k, C_{0}, C_{s}\left(\Omega, g_{0}\right)\right)\left|U^{-1}\right|_{k}\left(|U|_{\tilde{p}+1}+1\right) .
\end{aligned}
$$

Let $a_{k}=\left|U^{-1}\right|_{k}, c_{k}=|U|_{k}, b=\left(|U|_{\tilde{p}+1}+1\right)$. Then

$$
a_{k+1} \leq C\left(k, C_{0}, C_{s}\left(\Omega, g_{0}\right)\right) c_{k+1}+C\left(k, C_{0}, C_{s}\left(\Omega, g_{0}\right)\right) b a_{k} .
$$

By induction, it is easy to get

$$
a_{k+1} \leq C\left(k, C_{0}, C_{s}\left(\Omega, g_{0}\right)\right)(b+1)^{k+1}\left(c_{k+1}+1\right) .
$$

Hence

$$
\left|U^{-1}\right|_{k+1} \leq C\left(k, C_{0}, C_{s}\left(\Omega, g_{0}\right)\right)\left(|U|_{\tilde{p}+1}+1\right)^{k+1}\left(|U|_{k+1}+1\right) .
$$

Proposition 2.5. There exist some $t_{3}=t_{3}\left(n, \tilde{p}, G,\|\chi\|_{\tilde{p}+2},\|\widetilde{R m}\|_{\tilde{p}+2}, C_{s}\left(\Omega, g_{0}\right)\right)>$ 0 , such that $\Phi_{\epsilon}: \mathfrak{A}_{t_{3}} \cap \mathbb{B}_{\tilde{p}+2}(G)_{t_{3}} \rightarrow \mathfrak{A}_{t_{3}} \cap \mathbb{B}_{\tilde{p}+2}(G)_{t_{3}}$.

Proof. Choose $U \in \mathfrak{A}_{t_{1}} \cap \mathbb{B}_{\tilde{p}+2}(G)_{t_{1}}, t_{1}>0$, to be determined later; we first show that $V \in \mathfrak{A}_{t_{1}}$ if $V=\Phi_{\epsilon}(U)$.

Choose a positive integer $m$ such that $2^{m}>2 n$, and choose a normal coordinate system $\left\{x^{i}\right\}$, such that at one point

$$
\begin{gathered}
\left\{\tilde{g}_{i j}\right\}=\left(\begin{array}{cccc}
1 & & & \mathbf{0} \\
& 1 & & \\
& & \ddots & \\
\mathbf{0} & & & 1
\end{array}\right), \\
\left\{v_{i j}\right\}=\left(\begin{array}{cccc}
\lambda_{1} & & & \mathbf{0} \\
& \lambda_{2} & & \\
& & \ddots & \\
\mathbf{0} & & & \lambda_{n}
\end{array}\right),
\end{gathered}
$$

then

Now one can define

$$
\left\{v^{i j}\right\}=\left(\begin{array}{cccc}
\lambda_{1}^{-1} & & & \mathbf{0} \\
& \lambda_{2}^{-1} & & \\
& & \ddots & \\
\mathbf{0} & & & \lambda_{n}^{-1}
\end{array}\right) .
$$

$$
\phi_{m} \triangleq v^{\alpha_{1} \beta_{1}} \tilde{g}_{\beta_{1} \alpha_{2}} \cdots v^{\alpha_{m} \beta_{m}} \tilde{g}_{\beta_{m} \alpha_{1}}=\sum_{i=1}^{n} \lambda_{i}^{-m} .
$$


By (2.5) and $\frac{\partial}{\partial t} v^{i j}=-v^{i k} v^{j l} \frac{\partial}{\partial t} v_{k l}$,

$$
\frac{\partial}{\partial t} v^{i j}=-v^{i k} v^{j l}\left[A_{\epsilon}^{\alpha \beta}\left(v_{k l}\right)_{\alpha \beta}+D_{k l}(U, V)+B_{k l}(U, \tilde{\nabla} U)\right] .
$$

By (61) on page 236 of [12,

$$
\left(v^{i j}\right)_{\alpha \beta}=\tilde{\nabla}_{\alpha} \tilde{\nabla}_{\beta}\left(v^{i j}\right)=-v^{i k} v^{j l} \tilde{\nabla}_{\alpha} \tilde{\nabla}_{\beta}\left(v_{k l}\right)-\tilde{\nabla}_{\alpha}\left(v^{i k} v^{j l}\right) \tilde{\nabla}_{\beta} v_{k l} .
$$

By (2.7) and (2.8),

$$
\begin{aligned}
\frac{\partial}{\partial t} v^{i j}= & A_{\epsilon}^{\alpha \beta}\left(v^{i j}\right)_{\alpha \beta}+A_{\epsilon}^{\alpha \beta}\left(v^{i k} v^{j l}\right)_{\alpha}\left(v_{k l}\right)_{\beta}-v^{i k} v^{j l}\left[D_{k l}(U, V)+B_{k l}(U, \tilde{\nabla} U)\right] \\
\left(\phi_{m}\right)_{t}= & v^{\alpha_{1} \beta_{1}} \cdots\left(\frac{\partial}{\partial t} v^{\alpha_{k} \beta_{k}}\right) \cdots \tilde{g}_{\beta_{m} \alpha_{1}} \\
= & v^{\alpha_{1} \beta_{1}} \cdots\left[A_{\epsilon}^{\alpha \beta}\left(v^{\alpha_{k} \beta_{k}}\right)_{\alpha \beta}+A_{\epsilon}^{\alpha \beta}\left(v^{\alpha_{k} p} v^{\beta_{k} q}\right)_{\alpha}\left(v_{p q}\right)_{\beta}\right. \\
& \left.\quad-v^{\alpha_{k} p} v^{\beta_{k} q}\left[D_{p q}(U, V)+B_{p q}(U, \tilde{\nabla} U)\right]\right] \cdots \tilde{g}_{\beta_{m} \alpha_{1}} \\
= & A_{\epsilon}^{\alpha \beta}\left(\phi_{m}\right)_{\alpha \beta}+2 \lambda_{i}^{-m} \chi^{2} u^{\alpha \beta} R_{i \alpha i \beta}-\lambda_{i}^{-m-1} B_{i i}-2 A_{\epsilon}^{\alpha \beta} \lambda_{p}^{-m-1} \lambda_{q}^{-1}\left(v_{p q}\right)_{\alpha}\left(v_{p q}\right)_{\beta} \\
& -2 \sum_{k, l} \sum_{p<q} A_{\epsilon}^{\alpha \beta}\left(\lambda_{k}^{-1}\right)^{m+p-q-3}\left(\lambda_{l}^{-1}\right)^{q-p-3}\left(v_{k l}\right)_{\alpha}\left(v_{k l}\right)_{\beta}
\end{aligned}
$$

and

$$
\begin{aligned}
& -2 \sum_{k, l} \sum_{p<q} A_{\epsilon}^{\alpha \beta}\left(\lambda_{k}^{-1}\right)^{m+p-q-3}\left(\lambda_{l}^{-1}\right)^{q-p-3}\left(v_{k l}\right)_{\alpha}\left(v_{k l}\right)_{\beta} \\
= & -m \sum_{k, l} A_{\epsilon}^{\alpha \beta} \sum_{\sigma=-2}^{m-4}\left(\lambda_{k}^{-1}\right)^{m-\sigma-6}\left(\lambda_{l}^{-1}\right)^{\sigma}\left(v_{k l}\right)_{\alpha}\left(v_{k l}\right)_{\beta} .
\end{aligned}
$$

By (2.9) and (2.10),

$$
\begin{aligned}
\left(\phi_{m}\right)_{t} & =\left(\chi^{2}+\epsilon\right)\left(u^{\alpha \beta}\right)\left(\phi_{m}\right)_{\alpha \beta}-2\left(\lambda_{p}^{-1}\right)^{m+1} \lambda_{q}^{-1}\left(\chi^{2}+\epsilon\right) u^{\alpha \beta}\left(v_{p q}\right)_{\alpha}\left(v_{p q}\right)_{\beta} \\
& -m\left(\chi^{2}+\epsilon\right) \sum_{\sigma=-2}^{m-4} u^{\alpha \beta}\left(v_{k l}\right)_{\alpha}\left(v_{k l}\right)_{\beta}\left(\lambda_{k}^{-1}\right)^{m-\sigma-6}\left(\lambda_{l}^{-1}\right)^{\sigma} \\
& +2 \lambda_{i}^{-m} \chi^{2} u^{\alpha \beta} \tilde{R}_{i \alpha i \beta}-\lambda_{i}^{-m-1} B_{i i} ;
\end{aligned}
$$

then

$$
\left(\phi_{m}\right)_{t} \leq\left(\chi^{2}+\epsilon\right) u^{\alpha \beta}\left(\phi_{m}\right)_{\alpha \beta}+C(n) \chi^{2}|\widetilde{R m}| \phi_{m}-\lambda_{i}^{-m-1} B_{i i} .
$$

Because $U \in \mathfrak{A}_{t_{1}} \cap \mathbb{B}_{\tilde{p}+2}(G)_{t_{1}}$, by definition of $B_{i j}$ and (2.6),

$$
-\lambda_{i}^{-m-1} B_{i i} \leq C\left(n,\|\chi\|_{1}, C_{s}\left(\Omega, g_{0}\right)\right) \cdot(G+1)^{2} \phi_{m}^{1+\frac{1}{m}} .
$$

From (2.11), (2.12) and the Maximum Principle,

$$
\begin{gathered}
\left(\max _{x \in \Omega} \phi_{m}(x, t)\right)_{t} \leq C\left(n,\|\chi\|_{1},\|\widetilde{R m}\|_{0}, C_{s}\left(\Omega, g_{0}\right), G\right)\left(\max _{x \in \Omega} \phi_{m}(x, t)+1\right)^{2}, \\
\phi_{m}(x, t) \leq \frac{1}{C_{0}-C t}-1, \quad C_{0}=\frac{1}{n+1} .
\end{gathered}
$$

Note that $\phi_{m}(0)=2 n$. One can choose

$$
t_{1,1}=t_{1,1}\left(n,\|\chi\|_{1},\|\widetilde{R m}\|_{0}, C_{s}\left(\Omega, g_{0}\right), G\right)>0,
$$

such that when $t \in\left[0, t_{1,1}\right], \phi_{m}(x, t) \leq 2^{m}$, then

$$
\lambda_{i} \geq \frac{1}{2}, \quad i=1,2, \cdots, n \text {. }
$$


Now define

$$
\begin{aligned}
\psi_{m}= & v_{\alpha_{1} \beta_{1}} \tilde{g}^{\beta_{1} \alpha_{2}} \cdots v_{\alpha_{m} \beta_{m}} \tilde{g}^{\beta_{m} \alpha_{1}}=\sum_{i=1}^{n} \lambda_{i}^{m} \\
\left(\psi_{m}\right)_{t}= & \left(\chi^{2}+\epsilon\right) u^{\alpha \beta}\left(\psi_{m}\right)_{\alpha \beta}-2 \chi^{2} \lambda_{i}^{m} u^{\alpha \beta} \tilde{R}_{i \alpha i \beta}+\lambda_{i}^{m-1} B_{i i} \\
& -2\left(\chi^{2}+\epsilon\right) \sum_{j<k} \lambda_{q}^{m+j-k-1} \lambda_{r}^{k-j-1} u^{\alpha \beta}\left(v_{q r}\right)_{\alpha}\left(v_{q r}\right)_{\beta} \\
\leq & \left(\chi^{2}+\epsilon\right) u^{\alpha \beta}\left(\psi_{m}\right)_{\alpha \beta}+C\left(\psi_{m}+1\right)^{2} .
\end{aligned}
$$

Similarly, by (2.13) and the Maximum Principle,

$$
\phi_{m}(x, t) \leq \frac{1}{C_{0}-C t}-1, \quad C_{0}=\frac{1}{n+1} .
$$

Choose $t_{1,2}=t_{1,2}\left(n,\|\chi\|_{1},\|\widetilde{R m}\|_{0}, C_{s}\left(\Omega, g_{0}\right), G\right)>0$, such that when $t \in\left[0, t_{1,2}\right]$, $\psi_{m}(x, t) \leq 2^{m}$, then

$$
\lambda_{i} \leq 2 \quad i=1,2, \cdots, n .
$$

Set $t_{1}=\min \left\{t_{1,1}, t_{1,2}\right\}$ (note that $t_{1}=t_{1}\left(n,\|\chi\|_{1},\|\widetilde{R m}\|_{0}, C_{s}\left(\Omega, g_{0}\right), G\right)$ ). Then when $t \in\left[0, t_{1}\right], \frac{1}{2} \leq \lambda_{i} \leq 2$; hence $V \in \mathfrak{A}_{t_{1}}$. In fact we proved that $V \in \mathfrak{A}_{t_{1}}$ if $U \in \mathfrak{A}_{t_{1}} \cap \mathbb{B}_{\tilde{p}+2}(G)_{t_{1}}$ and $V=\Phi_{\epsilon}(U)$.

By (2.5), for $m \geq \tilde{p}+2$,

$$
\frac{\partial}{\partial t}|V|_{m}^{2}=2 \sum_{|\alpha| \leq m} \int_{\Omega}\left(\tilde{g}^{-1}\right)^{|\alpha|+2} \tilde{\nabla}^{\alpha} v_{k l} \tilde{\nabla}^{\alpha}\left(\frac{\partial}{\partial t} v_{i j}\right)=I_{1}+I_{2}+I_{3}+I_{4},
$$

where

$$
\begin{gathered}
I_{1}=2 \sum_{|\alpha| \leq m} \int_{\Omega}\left(\tilde{g}^{-1}\right)^{|\alpha|+2} \tilde{\nabla}^{\alpha} v_{k l} \tilde{\nabla}^{\alpha}\left(v_{i j}\right)_{p q} A_{\epsilon}^{p q}, \\
I_{2}=2 \sum_{|\alpha| \leq m} \int_{\Omega}\left(\tilde{g}^{-1}\right)^{|\alpha|+2} \tilde{\nabla}^{\alpha} v_{k l}\left[\tilde{\nabla}^{\alpha}\left(A_{\epsilon}^{p q}\left(v_{i j}\right)_{p q}\right)-A_{\epsilon}^{p q} \tilde{\nabla}^{\alpha}\left(v_{i j}\right)_{p q}\right], \\
I_{3}=2 \sum_{|\alpha| \leq m} \int_{\Omega}\left(\tilde{g}^{-1}\right)^{|\alpha|+2} \tilde{\nabla}^{\alpha} v_{k l} \tilde{\nabla}^{\alpha} D_{i j}, \quad I_{4}=2 \sum_{|\alpha| \leq m} \int_{\Omega}\left(\tilde{g}^{-1}\right)^{\mid \alpha+2} \tilde{\nabla}^{\alpha} v_{k l} \tilde{\nabla}^{\alpha} B_{i j}, \\
2.15) I_{1} \leq-2 \sum_{|\alpha| \leq m} \int\left(\tilde{g}^{-1}\right)^{|\alpha|+2}\left(\left(\tilde{\nabla}^{\alpha} v_{k l}\right)_{p} \tilde{\nabla}^{\alpha}\left(v_{i j}\right)_{q} A_{\epsilon}+\left(\tilde{\nabla}^{\alpha} v_{k l}\right) \tilde{\nabla}^{\alpha}\left(v_{i j}\right)_{q}\left(A_{\epsilon}\right)_{p}\right) \\
\leq-\frac{1}{2} \sum_{|\alpha|=m} \int\left(\chi^{2}+\epsilon\right)\left|\tilde{\nabla}^{\alpha+1} V\right|^{2}+C\left(n, m, G, C_{s}\left(\Omega, g_{0}\right),|| \chi \|_{1}\right)|V|_{m}^{2},
\end{gathered}
$$

where we used the fact that $\left|\tilde{\nabla} U^{-1}\right| \leq\left|U^{-1}\right|^{2}|\tilde{\nabla} U| \leq C\left(G, C_{s}\left(\Omega, g_{0}\right)\right)$. Then

$$
\begin{gathered}
I_{2} \leq 2 \sum_{|\alpha| \leq m}\left|\tilde{\nabla}^{\alpha} V\right|_{L^{2}} \cdot\left[C\left(\|\chi\|_{1}\right)\left(\int\left(\chi^{2}+\epsilon\right)\left|\tilde{\nabla}^{\alpha+1} V\right|^{2}\right)^{\frac{1}{2}}\right. \\
\left.+\left|\tilde{\nabla}^{\alpha-2}\left[\tilde{\nabla}^{2} A_{\epsilon}^{p q}(V)_{p q}\right]\right|_{L^{2}}\right] .
\end{gathered}
$$

By interpolation inequality, it is easy to get

$$
\begin{aligned}
\left|\tilde{\nabla}^{\alpha-2}\left[\tilde{\nabla}^{2} A^{p q}(V)_{p q}\right]\right|_{L^{2}} & \leq C\left(\|\chi\|_{2}, m\right)|V|_{m} \\
& +C\left(m, C_{s}\left(\Omega, g_{0}\right)\right)\left(|V|_{\tilde{p}+2}+1\right)\left(\left|A_{\epsilon}^{p q}\right|_{m}+1\right) .
\end{aligned}
$$

From Lemma 2.4 and $\left|U^{-1}\right|_{L^{\infty}} \leq 2 n$,

$$
\left|U^{-1}\right|_{k+1} \leq C\left(k, C_{s}\left(\Omega, g_{0}\right)\right)\left(|U|_{\tilde{p}+1}+1\right)^{k+1}\left(|U|_{k+1}+1\right) \text {. }
$$


Hence

$$
\begin{aligned}
\left|\tilde{\nabla}^{\alpha-2}\left[\tilde{\nabla}^{2} A^{p q}(V)_{p q}\right]\right|_{L^{2}} & \leq C\left(\|\chi\|_{2}, m\right)|V|_{m} \\
& +C\left(m, C_{s}\left(\Omega, g_{0}\right)\right)\left(|V|_{\tilde{p}+2}+1\right)\left(|U|_{m}+1\right) .
\end{aligned}
$$

By (2.16) and (2.17),

$$
\begin{aligned}
& I_{2} \leq \frac{1}{16} \sum_{|\alpha|=m} \int\left(\chi^{2}+\epsilon\right)\left|\tilde{\nabla}^{\alpha+1} V\right|^{2}+C\left(\|\chi\|_{1}\right)|V|_{m}^{2} \\
& +C\left(m,|| \chi \|_{2}, C_{s}\left(\Omega, g_{0}\right)\right)|V|_{m}\left(|V|_{\tilde{p}+2}+1\right)\left(|U|_{m}+1\right) .
\end{aligned}
$$

Similar to the estimate of $I_{2}$,

$$
\begin{gathered}
I_{3} \leq C\left(m,\|\chi\|_{m},\|\widetilde{R m}\|_{m}, C_{s}\left(\Omega, g_{0}\right)\right)\left(|V|_{m}^{2}+|V|_{m}\left(|V|_{\tilde{p}}+1\right)\left(|U|_{m}+1\right)\right), \\
I_{4}=I_{5}+I_{6}
\end{gathered}
$$

where

$$
\begin{aligned}
& I_{5}=2 \sum_{|\alpha| \leq m} \int\left(\tilde{g}^{-1}\right)^{|\alpha|+2} \tilde{\nabla}^{\alpha} v_{k l} \tilde{\nabla}^{\alpha} E_{i j}, \\
& I_{6}=2 \sum_{|\alpha| \leq m} \int\left(\tilde{g}^{-1}\right)^{|\alpha|+2} \tilde{\nabla}^{\alpha} v_{k l} \tilde{\nabla}^{\alpha} F_{i j}
\end{aligned}
$$

for $I_{5}$. We only estimate one term of it as in the following; the rest are similar:

$$
\begin{aligned}
& 2 \sum_{|\alpha| \leq m} \int\left(\tilde{g}^{-1}\right)^{|\alpha|+2} \tilde{\nabla}^{\alpha} v_{k l} \tilde{\nabla}^{\alpha}\left[\frac{1}{2} \chi^{2} u^{r s} u^{p q}\left(u_{r p}\right)_{i}\left(u_{s q}\right)_{j}\right] \\
& \leq C(n) \sum_{\alpha}\left[\left|\tilde{\nabla}^{\alpha} V\right|_{L^{2}} \cdot\left|\tilde{\nabla}^{\alpha-1}\left[\tilde{\nabla}\left(\chi^{2} U^{-2}\right) \cdot(\tilde{\nabla} U)^{2}\right]\right|_{L^{2}}\right] \\
&+C(n) \sum_{\alpha} \int \tilde{\nabla}^{\alpha} V \cdot\left(\chi^{2} U^{-2}\right) \tilde{\nabla}^{\alpha}(\tilde{\nabla} U \tilde{\nabla} U) \\
& \leq C\left(n, m,|| \chi||_{m}, C_{s}\left(\Omega, g_{0}\right)\right)|V|_{m} \\
& \cdot\left[\left|U^{-2}\right|_{m}\left|\left(\left.(\tilde{\nabla} U)^{2}\right|_{\tilde{p}}+1\right)+\left(\left|U^{-2}\right|_{\tilde{p}+1} \mid+1\right)(\tilde{\nabla} U)^{2}\right|_{m-1}\right] \\
&+ C(n) \sum_{\alpha} \int\left|\tilde{\nabla} \alpha+1 V \cdot\left(\chi^{2} U^{-2}\right) \tilde{\nabla}^{\alpha-1}(\tilde{\nabla} U \tilde{\nabla} U)\right| \\
&+C(n) \sum_{\alpha} \int\left|\tilde{\nabla}^{\alpha} V \cdot \tilde{\nabla}\left(\chi^{2} U^{-2}\right) \tilde{\nabla}^{\alpha-1}(\tilde{\nabla} U \tilde{\nabla} U)\right| \\
& \leq \frac{1}{64} \sum_{|\alpha|=m} \int \chi^{2}\left|\tilde{\nabla}^{\alpha+1} V\right|^{2}+C\left(n, m,|| \chi \|_{m}, C_{s}\left(\Omega, g_{0}\right), G\right) \\
& \cdot\left(|V|_{m}\left(|U|_{m}+1\right)+|U|_{m}^{2}+|V|_{m}|U|_{m}\right) .
\end{aligned}
$$

Hence

$$
\begin{aligned}
I_{5} \leq & \frac{9}{64} \sum_{|\alpha|=m} \int \chi^{2}\left|\tilde{\nabla}^{\alpha+1} V\right|^{2}+C\left(n, m,\|\chi\|_{m}, C_{s}\left(\Omega, g_{0}\right), G\right) \\
& \cdot\left(|V|_{m}\left(|U|_{m}+1\right)+|U|_{m}^{2}\right)
\end{aligned}
$$


for $I_{6}$. We only estimate one term of it as in the following; the rest are similar:

$$
\begin{aligned}
& 2 \sum_{|\alpha| \leq m} \int\left(\tilde{g}^{-1}\right)^{|\alpha|+2} \tilde{\nabla}^{\alpha} v_{k l} \tilde{\nabla}^{\alpha}\left[\chi \chi_{i} u^{p q}\left(u_{j q}\right)_{p}\right] \\
& \leq C(n) \sum_{\alpha} \int \tilde{\nabla}^{\alpha} V \cdot \tilde{\nabla}^{\alpha-1}\left[\tilde{\nabla}\left(\chi \tilde{\nabla} \chi U^{-1}\right) \cdot(\tilde{\nabla} U)\right] \\
& \quad+2 \sum_{\alpha} \int \tilde{\nabla}^{\alpha} V \cdot\left(\chi \tilde{\nabla} \chi U^{-1}\right) \tilde{\nabla}^{\alpha+1} U \\
& \leq \frac{1}{64} \sum_{|\alpha|=m} \int \chi^{2}\left|\tilde{\nabla}^{\alpha+1} V\right|^{2}+C\left(n, m, G,\|\chi\|_{m}, C_{s}\left(\Omega, g_{0}\right)\right) \\
& \quad \cdot\left(|V|_{m}\left(|U|_{m}+1\right)+|U|_{m}^{2}+|V|_{m}|U|_{m}\right) .
\end{aligned}
$$

Hence

$$
\begin{aligned}
I_{6} \leq & \frac{6}{64} \sum_{|\alpha|=m} \int \chi^{2}\left|\tilde{\nabla}^{\alpha+1} V\right|^{2}+C\left(n, m, G,\|\chi\|_{m}, C_{s}\left(\Omega, g_{0}\right)\right) \\
& \cdot\left(|U|_{m}^{2}+|V|_{m}\left(|U|_{m}+1\right)\right) .
\end{aligned}
$$

Then we get

$$
\begin{aligned}
I_{4} \leq & \frac{15}{64} \sum_{|\alpha|=m} \int \chi^{2}\left|\tilde{\nabla}^{\alpha+1} V\right|^{2}+C\left(n, m, G,\|\chi\|_{m}, C_{s}\left(\Omega, g_{0}\right)\right) \\
& \cdot\left(|U|_{m}^{2}+|V|_{m}\left(|U|_{m}+1\right)\right) .
\end{aligned}
$$

By (2.14), (2.15), (2.18), (2.19) and (2.21), and noting that the sum of the coefficients of $\sum_{|\alpha| \leq m} \int \chi^{2}\left|\tilde{\nabla}^{\alpha+1} V\right|^{2}$ is negative on the right side, then

$$
\frac{\partial}{\partial t}|V|_{m}^{2} \leq C\left(|V|_{m}^{2}+|V|_{m}\left(|V|_{\tilde{p}+2}+1\right)\left(|U|_{m}+1\right)+|U|_{m}^{2}\right)
$$

where $C=C\left(n, m, G, C_{s}\left(\Omega, g_{0}\right),\|\chi\|_{m},\|\widetilde{R m}\|_{m}\right)$. For simplicity, we use $C$ instead of $C\left(n, m, G, C_{s}\left(\Omega, g_{0}\right),\|\chi\|_{m},\|\widehat{R m}\|_{m}\right)$ in the rest of the proof. Let $\phi(t)=|V|_{m}^{2}(t)$. Then

$$
\phi_{t} \leq C \phi+C \phi^{\frac{1}{2}} Q_{1}+Q_{2}
$$

where $Q_{1}=\left(|V|_{\tilde{p}+2}+1\right)\left(|U|_{m}+1\right)$ and $Q_{2}=C|U|_{m}^{2}$. We can get

$$
\phi_{t} \leq C(\phi+1)\left(Q_{1}^{2}+Q_{2}+1\right) \leq C Q_{4}(\phi+1),
$$

where

$$
Q_{4}=\left(|V|_{\tilde{p}+2}^{2}+1\right)\left(|U|_{m}+1\right)^{2} .
$$

Choose $m=\tilde{p}+2, \phi(t)=|V|_{\tilde{p}+2}^{2}(t)$, and define $\psi(t)=|U|_{\tilde{p}+2}(t)$. Then $Q_{4}=$ $(\phi+1)(\psi+1)^{2}$,

$$
\phi_{t} \leq C(\phi+1)^{2}(\psi+1)^{2} \leq C(G+1)^{2}(\phi+1)^{2}=C(\phi+1)^{2},
$$

where $\psi \leq G$ because of $U \in \mathbb{B}_{\tilde{p}+2}(G)_{t_{1}}$ and

$$
\begin{gathered}
C=C\left(n, \tilde{p}, G,\|\chi\|_{\tilde{p}+2}\|\widetilde{R m}\|_{\tilde{p}+2}, C_{s}\left(\Omega, g_{0}\right)\right), \\
\phi(t) \leq \frac{1}{C_{0}-C t}-1, \quad C_{0}=(\phi(0)+1)^{-1} .
\end{gathered}
$$


Note that $\phi(0)=\left(\frac{G}{2}\right)^{2}$. Then there exists $t_{2}>0$, such that if $t \in\left[0, t_{2}\right]$, $\phi(t) \leq G^{2}$; that is

$$
|V|_{\tilde{p}+2} \leq G
$$

Choose

$$
t_{3}=\min \left\{t_{1}, t_{2}\right\}=t_{3}\left(n, \tilde{p}, G,\|\chi\|_{\tilde{p}+2},\|\widetilde{R m}\|_{\tilde{p}+2}, C_{s}\left(\Omega, g_{0}\right)\right) .
$$

We get the a priori estimate that $V \in \mathfrak{A}_{t_{3}} \cap \mathbb{B}_{\tilde{p}+2}(G)_{t_{3}}$ if $U \in \mathfrak{A}_{t_{3}} \cap \mathbb{B}_{\tilde{p}+2}(G)_{t_{3}}$ and $V=\Phi_{\epsilon}(U)$. By Theorem 8.3 in [9] (which is for parabolic equations, but similar results apply for the parabolic system here), we in fact get the solution of (2.4) on $\Omega \times\left[0, t_{3}\right]$ and

$$
\Phi_{\epsilon}: \mathfrak{A}_{t_{3}} \cap \mathbb{B}_{\tilde{p}+2}(G)_{t_{3}} \longrightarrow \mathfrak{A}_{t_{3}} \cap \mathbb{B}_{\tilde{p}+2}(G)_{t_{3}} .
$$

Remark 2.6. The proof of Proposition 2.5 (especially the energy estimate) has the same spirit as in [7, but the tensor version makes our estimate more subtle. Also note that $t_{3}$ is independent of $\epsilon$.

Proposition 2.7. For any $m \geq \tilde{p}+2$, there exists

$$
t_{5}=t_{5}\left(n, m, G,\|\chi\|_{m},\|\widetilde{R m}\|_{m}, C_{s}\left(\Omega, g_{0}\right)\right)>0
$$

such that

$$
\Phi_{\epsilon}: \mathfrak{A}_{t_{5}} \cap \mathbb{B}_{m}(G)_{t_{5}} \longrightarrow \mathfrak{A}_{t_{5}} \cap \mathbb{B}_{m}(G)_{t_{5}} .
$$

Proof. Choose $U \in \mathfrak{A}_{T} \cap \mathbb{B}_{m}(G)_{T}$, where $T \leq t_{3}$ is to be determined later. By Proposition 2.5, we get the solution $V=\Phi_{\epsilon}(U) \in \mathfrak{A}_{T} \cap \mathbb{B}_{\tilde{p}+2}(G)_{T}$, so $|V|_{\tilde{p}+2} \leq G$. We also have $|U|_{m} \leq G$. By (2.23) and (2.24) we get

$$
\phi_{t} \leq C\left(G^{2}+1\right)(G+1)^{2}(\phi+1) \leq C(\phi+1),
$$

where $\phi(t)=|V|_{m}^{2}(t)$ and $C=C\left(n, m, G,\|\chi\|_{m},\|\widetilde{R m}\|_{m}, C_{s}\left(\Omega, g_{0}\right)\right)$. Then

$$
\phi(t) \leq e^{C t}[\phi(0)+1]-1 \text {. }
$$

Note $\phi(0)=\left(\frac{G}{2}\right)^{2}$. Choose $t_{4}=t_{4}\left(n, m, G,\|\chi\|_{m},\|\widetilde{R m}\|_{m}, C_{s}\left(\Omega, g_{0}\right)\right)>0$ such that $e^{C t_{4}}[\phi(0)+1]-1=G^{2}$, and let

$$
t_{5}=\min t_{3}, t_{4}=t_{5}\left(n, m, G,\|\chi\|_{m},\|\widetilde{R m}\|_{m}, C_{s}\left(\Omega, g_{0}\right)\right) \text {. }
$$

If $t \in\left[0, t_{5}\right]$,

$$
\phi(t) \leq e^{C t_{5}}\left[\left(\frac{G}{2}\right)^{2}+1\right]-1 \leq G^{2} ;
$$

then $|V|_{m} \leq G$. Hence $V \in \mathfrak{A}_{t_{5}} \cap \mathbb{B}_{m}(G)_{t_{5}}$.

Next we show that $\Phi_{\epsilon}$ is a contraction map.

Proposition 2.8. For $0<\delta<1$, there exists

$$
t_{7}=t_{7}\left(n, \tilde{p}, G,\|\chi\|_{\tilde{p}+2},\|\widetilde{R m}\|_{\tilde{p}+2}, C_{s}\left(\Omega, g_{0}\right), \delta\right)>0
$$

such that

$$
\left|\Phi_{\epsilon}(U)-\Phi_{\epsilon}(\tilde{U})\right|_{t_{7}, \tilde{p}} \leq \delta|U-\tilde{U}|_{t_{7}, \tilde{p}}
$$

where $U, \tilde{U} \in \mathfrak{A}_{t_{7}} \cap \mathbb{B}_{\tilde{p}+2}(G)_{t_{7}}$. 
Proof. By (2.5) we have the following:

$$
\left\{\begin{array}{l}
V_{t}=\left(\chi^{2}+\epsilon\right) u^{\alpha \beta}(V)_{\alpha \beta}+P(U) V+B(U, \tilde{\nabla} U) \\
\tilde{V}_{t}=\left(\chi^{2}+\epsilon\right) \tilde{u}^{\alpha \beta}(\tilde{V})_{\alpha \beta}+P(\tilde{U}) \tilde{V}+B(\tilde{U}, \tilde{\nabla} \tilde{U})
\end{array}\right.
$$

where

$$
\begin{gathered}
V=\Phi_{\epsilon}(U),(P(U) V)_{i j}=D_{i j}(U, V), \\
(B(U, \tilde{\nabla} U))_{i j}=B_{i j}(U, \tilde{\nabla} U),(V)_{\alpha \beta}=\tilde{\nabla}_{\alpha} \tilde{\nabla}_{\beta} V .
\end{gathered}
$$

There is a similar notation for $\tilde{V}$, etc. By Proposition 2.5 if $T \leq t_{3}$, where $t_{3}$ is defined in (2.25), then $V, \tilde{V} \in \mathfrak{A}_{T} \cap \mathbb{B}_{\tilde{p}+2}(G)_{T}$.

Define $W \triangleq V-\tilde{V}=\Phi_{\epsilon}(U)-\Phi_{\epsilon}(\tilde{U})$. Then

$$
W_{t}=\left(\chi^{2}+\epsilon\right) u^{\alpha \beta} w_{\alpha \beta}+P(U) W+H,
$$

where

$$
H=\left(\chi^{2}+\epsilon\right)\left(u^{\alpha \beta}-\tilde{u}^{\alpha \beta}\right)(\tilde{V})_{\alpha \beta}+(P(U)-P(\tilde{U})) \tilde{V}+(B(U, \tilde{\nabla} U)-B(\tilde{U}, \tilde{\nabla} \tilde{U})) .
$$

We consider the evolution equation of $|W|_{\tilde{p}}^{2}$ :

$$
\frac{\partial}{\partial t}|W|_{\tilde{p}}^{2}=J_{1}+J_{2}+J_{3}+J_{4}
$$

where

$$
\begin{gathered}
J_{1}=2 \sum_{|\alpha| \leq \tilde{p}} \int\left(\tilde{g}^{-1}\right)^{|\alpha|+2} \tilde{\nabla}^{\alpha} w_{k l} \tilde{\nabla}^{\alpha}\left(w_{i j}\right)_{p q} A_{\epsilon}^{p q} \\
J_{2}=2 \sum \int\left(\tilde{g}^{-1}\right)^{|\alpha|+2} \tilde{\nabla}^{\alpha} w_{k l}\left[\tilde{\nabla}^{\alpha}\left(A_{\epsilon}^{p q}\left(w_{i j}\right)_{p q}\right)-A_{\epsilon}^{p q} \tilde{\nabla}^{\alpha}\left(w_{i j}\right)_{p q}\right] \\
J_{3}=2 \sum_{|\alpha| \leq \tilde{p}} \int\left(\tilde{g}^{-1}\right)^{|\alpha|+2} \tilde{\nabla}^{\alpha} w_{k l} \tilde{\nabla}^{\alpha}(P(U) W)_{i j}, \\
J_{4}=2 \sum_{|\alpha| \leq \tilde{p}} \int\left(\tilde{g}^{-1}\right)^{|\alpha|+2} \tilde{\nabla}^{\alpha} w_{k l} \tilde{\nabla}^{\alpha} H_{i j} .
\end{gathered}
$$

Recall $A_{\epsilon}^{p q}=\left(\chi^{2}+\epsilon\right) u^{p q}$. Similar to (2.15), we have

$$
J_{1} \leq-\frac{1}{2} \sum_{|\alpha|=\tilde{p}} \int\left(\chi^{2}+\epsilon\right)\left|\tilde{\nabla}^{\alpha+1} W\right|^{2}+C\left(n, \tilde{p}, G, C_{s}\left(\Omega, g_{0}\right),\|\chi\|_{1}\right)|W|_{\tilde{p}}^{2} .
$$

Similar to (2.16), we get

$$
J_{2} \leq 2 \sum_{|\alpha| \leq \tilde{p}}\left|\tilde{\nabla}^{\alpha} W\right|_{L^{2}} \cdot\left[C\left(\int\left(\chi^{2}+\epsilon\right)\left|\tilde{\nabla}^{\alpha+1} W\right|^{2}\right)^{\frac{1}{2}}+\left|\tilde{\nabla}^{\alpha-2}\left[\nabla^{2} A_{\epsilon}^{p q}(W)_{p q}\right]\right|_{L^{2}}\right],
$$

and we have

$$
\begin{gathered}
\left|\tilde{\nabla}^{\alpha-2}\left[\tilde{\nabla}^{2} A_{\epsilon}^{p q}(W)_{p q}\right]\right|_{L^{2}} \leq C(\tilde{p}) \sum_{\beta+\gamma=\alpha}\left|\tilde{\nabla}^{\beta}\left(\tilde{\nabla}^{2} A^{p q}\right) \tilde{\nabla}^{\gamma} W\right|_{L^{2}} \\
\leq C\left(n, \tilde{p}, C_{s}\left(\Omega, g_{0}\right)\right)\left[\left(\left|\tilde{\nabla}^{2} A_{\epsilon}^{p q}\right|_{L^{\infty}}+1\right)\left|\tilde{\nabla}{ }^{\alpha} W\right|_{L^{2}}+|W|_{L^{\infty}}\left(\left|\tilde{\nabla}^{\alpha}\left(\tilde{\nabla}^{2} A_{\epsilon}^{p q}\right)\right|_{L^{2}}+1\right)\right] \\
\leq C\left(n, \tilde{p}, C_{s}\left(\Omega, g_{0}\right),\left.|| \chi\right|_{2}\right)\left[\left(|U|_{\tilde{p}+2}+1\right)|W|_{\tilde{p}}+|W|_{\tilde{p}}\left(|U|_{\tilde{p}+2}+1\right)\right] \\
\leq C\left(n, \tilde{p}, C_{s}\left(\Omega, g_{0}\right),\left.|| \chi\right|_{2}, G\right)|W|_{\tilde{p}} .
\end{gathered}
$$


Hence

$$
J_{2} \leq \frac{1}{16} \sum_{|\alpha|=\tilde{p}} \int\left(\chi^{2}+\epsilon\right)\left|\tilde{\nabla}^{\alpha+1} W\right|^{2}+C\left(n, \tilde{p}, C_{s}\left(\Omega, g_{0}\right),\|\chi\|_{2}, G\right)|W|_{\tilde{p}}^{2}
$$

Similar to (2.19), we get

$$
J_{3} \leq C\left(\tilde{p},\|\chi\|_{\tilde{p}},\|\widetilde{R m}\|_{\tilde{p}}, C_{s}\left(\Omega, g_{0}\right), G\right)|W|_{\tilde{p}}^{2} .
$$

In the rest of the proof, we use $C$ instead of $C\left(n, \tilde{p},\|\chi\|_{\tilde{p}},\|\widetilde{R m}\|_{\tilde{p}}, C_{s}\left(\Omega, g_{0}\right), G\right)$ for simplicity. We have

$$
J_{4}=J_{5}+J_{6}+J_{7}
$$

where

$$
\begin{gathered}
J_{5}=2 \sum_{\alpha} \int\left(\tilde{g}^{-1}\right)^{\alpha+2} \tilde{\nabla}^{\alpha} W \cdot \tilde{\nabla}^{\alpha}\left[\left(\chi^{2}+\epsilon\right)\left(U^{-1}-\tilde{U}^{-1}\right) \tilde{\nabla}^{2} \tilde{V}\right] \\
J_{6}=2 \sum_{\alpha} \int\left(\tilde{g}^{-1}\right)^{\alpha+2} \tilde{\nabla}^{\alpha} W \cdot \tilde{\nabla}^{\alpha}[(P(U)-P(\tilde{U})) \tilde{V}] \\
J_{7}=2 \sum_{\alpha} \int\left(\tilde{g}^{-1}\right)^{\alpha+2} \tilde{\nabla}^{\alpha} W \cdot \tilde{\nabla}^{\alpha}[B(U, \tilde{\nabla} U)-B(\tilde{U}-\tilde{\nabla} \tilde{U})] \\
J_{5} \leq C|W|_{\tilde{p}}\left(\sum_{\alpha}\left|\tilde{\nabla}^{\alpha}\left[\left(\chi^{2}+\epsilon\right) \tilde{\nabla}^{2} \tilde{V} \cdot\left(U^{-1}-\tilde{U}^{-1}\right)\right]\right|_{L^{2}}\right) \\
\leq C|W|_{\tilde{p}}\left(|\tilde{V}|_{\tilde{p}+2}+1\right)\left|U^{-1}-\tilde{U}^{-1}\right|_{\tilde{p}} \leq C|W|_{\tilde{p}}|\tilde{U}-U|_{\tilde{p}}
\end{gathered}
$$

It is easy to get

$$
J_{6} \leq C|W|_{\tilde{p}}\left[|P(U)-P(\tilde{U})|_{\tilde{p}}\left(|\tilde{V}|_{\tilde{p}}+1\right)\right] \leq C|W|_{\tilde{p}}|\tilde{U}-U|_{\tilde{p}}
$$

We only need to estimate two terms of $J_{7}$ such as in the following for $J_{8}$ and $J_{9}$; the others are similar:

$$
\begin{gathered}
J_{8}=\sum_{\alpha} \int\left(\tilde{g}^{-1}\right)^{|\alpha|+2} \cdot \tilde{\nabla}^{\alpha} W \cdot \tilde{\nabla}^{\alpha}\left[\chi^{2}\left(U^{-2}(\tilde{\nabla} U)^{2}-\tilde{U}^{-2}(\tilde{\nabla} \tilde{U})^{2}\right)\right], \\
J_{9}=\sum_{\alpha} \int\left(\tilde{g}^{-1}\right)^{|\alpha|+2} \cdot \tilde{\nabla}^{\alpha} W \cdot \tilde{\nabla}^{\alpha}\left[\chi \tilde{\nabla} \chi\left(U^{-1} \tilde{\nabla} U-\tilde{U}^{-1} \tilde{\nabla} \tilde{U}\right)\right] .
\end{gathered}
$$

We first estimate $J_{8}$ :

$$
J_{8} \leq J_{10}+J_{11}+J_{12}+J_{13}
$$

where

$$
\begin{gathered}
J_{10}=C \sum_{\alpha} \int\left(\tilde{g}^{-1}\right)^{|\alpha|+2} \cdot \tilde{\nabla}^{\alpha} W \cdot \tilde{\nabla}^{\alpha}\left[\left(U^{-1}-\tilde{U}^{-1}\right) U^{-1}(\tilde{\nabla} U)^{2} \chi^{2}\right], \\
J_{11}=C \sum_{\alpha} \int\left(\tilde{g}^{-1}\right)^{|\alpha|+2} \cdot \tilde{\nabla}^{\alpha} W \cdot \tilde{\nabla}^{\alpha}\left[\left(U^{-1}-\tilde{U}^{-1}\right) \tilde{U}^{-1}(\tilde{\nabla} U)^{2} \chi^{2}\right], \\
J_{12}=C \sum_{\alpha} \int\left(\tilde{g}^{-1}\right)^{|\alpha|+2} \cdot \tilde{\nabla}^{\alpha} W \cdot \tilde{\nabla}^{\alpha}\left[\left(\tilde{U}^{-2} \tilde{\nabla} U \chi^{2}\right) \tilde{\nabla}(U-\tilde{U})\right], \\
J_{13}=C \sum_{\alpha} \int\left(\tilde{g}^{-1}\right)^{|\alpha|+2} \cdot \tilde{\nabla}^{\alpha} W \cdot \tilde{\nabla}^{\alpha}\left[\left(\tilde{U}^{-2} \tilde{\nabla} \tilde{U} \chi^{2}\right) \tilde{\nabla}(U-\tilde{U})\right] .
\end{gathered}
$$

It is easy to get

$$
J_{10} \leq C|W|_{\tilde{p}}\left[\left|U^{-1}-\tilde{U}^{-1}\right|_{\tilde{p}}\left(\left|U^{-1}(\nabla U)^{2}\right|_{\tilde{p}}+1\right)\right] \leq C|W|_{\tilde{p}}|\tilde{U}-U|_{\tilde{p}}
$$


Similarly, we get

$$
J_{11} \leq C|W|_{\tilde{p}}|\tilde{U}-U|_{\tilde{p}}
$$

We have $J_{12} \leq J_{12.1}+J_{12.2}$, where

$$
\begin{gathered}
J_{12.1}=C \sum_{\alpha} \int\left(\tilde{g}^{-1}\right)^{|\alpha|+2} \cdot \tilde{\nabla}^{\alpha} W \cdot \tilde{\nabla}^{\alpha-1}\left[\tilde{\nabla}\left(\tilde{U}^{-2} \tilde{\nabla} U \chi^{2}\right) \tilde{\nabla}(U-\tilde{U})\right] \\
J_{12.2}=C \sum_{\alpha} \int\left(\tilde{g}^{-1}\right)^{|\alpha|+2} \cdot \tilde{\nabla}^{\alpha} W \cdot\left(\tilde{U}^{-2} \tilde{\nabla} U \chi^{2}\right) \cdot \tilde{\nabla}^{\alpha+1}(U-\tilde{U}), \\
J_{12.1} \leq C|W|_{\tilde{p}} \sum_{\alpha}\left|\tilde{\nabla}^{\alpha-1}[f \tilde{\nabla} g]\right|_{L^{2}},
\end{gathered}
$$

where $f=\tilde{\nabla}\left(\tilde{U}^{-2} \chi^{2} \tilde{\nabla} U\right), g=U-\tilde{U}$. Note that $|f|_{\tilde{p}} \leq C\left(|u|_{\tilde{p}+2}+|\tilde{u}|_{\tilde{p}+2}+1\right) \leq C$.

Then

$$
\begin{aligned}
\left|\tilde{\nabla}^{\alpha-1}[f \tilde{\nabla} g]\right|_{L^{2}} & \leq C \sum_{\beta+\gamma=\alpha}\left|\tilde{\nabla}^{\beta} f \tilde{\nabla}^{\gamma} g\right|_{L^{2}} \\
& \leq C\left(\left(|f|_{L^{\infty}}+1\right)\left|\tilde{\nabla}^{\alpha} g\right|_{L^{2}}+|g|_{L^{\infty}}\left(\left|\tilde{\nabla}^{\alpha} f\right|_{L^{2}}+1\right)\right) \\
& \leq C|g|_{\tilde{p}}=C|U-\tilde{U}|_{\tilde{p}} .
\end{aligned}
$$

With (2.36) and (2.37), we get

$$
J_{12.1} \leq C|W|_{\tilde{p}}|U-\tilde{U}|_{\tilde{p}}
$$

It is easy to get

$$
J_{12.2} \leq \frac{1}{1000} \sum_{|\alpha|=\tilde{p}} \int\left|\tilde{\nabla}^{\alpha+1} W\right|^{2} \chi^{2}+C|U-\tilde{U}|_{\tilde{p}}^{2}+C|W|_{\tilde{p}}|U-\tilde{U}|_{\tilde{p}},
$$

and by (2.38) and (2.39),

$$
J_{12} \leq \frac{1}{1000} \sum_{|\alpha|=\tilde{p}} \int\left|\tilde{\nabla}^{\alpha+1} W\right|^{2} \chi^{2}+C|U-\tilde{U}|_{\tilde{p}}^{2}+C|W|_{\tilde{p}}|U-\tilde{U}|_{\tilde{p}} .
$$

Similarly,

$$
J_{13} \leq \frac{1}{1000} \sum_{|\alpha|=\tilde{p}} \int\left|\tilde{\nabla}^{\alpha+1} W\right|^{2} \chi^{2}+C|U-\tilde{U}|_{\tilde{p}}^{2}+C|W|_{\tilde{p}}|U-\tilde{U}|_{\tilde{p}} .
$$

By (2.34), (2.35), (2.40), (2.41) and (2.33),

$$
\begin{gathered}
J_{8} \leq \frac{1}{500} \sum_{|\alpha|=\tilde{p}} \int\left|\tilde{\nabla}^{\alpha+1} W\right|^{2} \chi^{2}+C|U-\tilde{U}|_{\tilde{p}}^{2}+C|W|_{\tilde{p}}|U-\tilde{U}|_{\tilde{p}} \\
J_{9} \leq J_{14}+J_{15}
\end{gathered}
$$

where

$$
\begin{aligned}
& J_{14}=\sum_{\alpha} \int\left(\tilde{g}^{-1}\right)^{|\alpha|+2} \cdot \tilde{\nabla}^{\alpha} W \cdot \tilde{\nabla}^{\alpha}\left[(\chi \tilde{\nabla} \chi \tilde{\nabla} U)\left(U^{-1}-\tilde{U}^{-1}\right)\right], \\
& J_{15}=\sum_{\alpha} \int\left(\tilde{g}^{-1}\right)^{|\alpha|+2} \cdot \tilde{\nabla}^{\alpha} W \cdot \tilde{\nabla}^{\alpha}\left[\left(\chi \tilde{\nabla} \chi \tilde{U}^{-1}\right) \tilde{\nabla}(U-\tilde{U})\right] .
\end{aligned}
$$

It is easy to get

$$
J_{14} \leq C|W|_{\tilde{p}}|U-\tilde{U}|_{\tilde{p}}
$$


Similar to the argument for $J_{12}$, we can get

$$
J_{15} \leq \frac{1}{1000} \sum_{|\alpha|=\tilde{p}} \int\left|\tilde{\nabla}^{\alpha+1} W\right|^{2} \chi^{2}+C|U-\tilde{U}|_{\tilde{p}}^{2}+C|W|_{\tilde{p}}|U-\tilde{U}|_{\tilde{p}} .
$$

By (2.44), (2.45) and (2.43), we get

$$
J_{9} \leq \frac{1}{1000} \sum_{|\alpha|=\tilde{p}} \int\left|\tilde{\nabla}^{\alpha+1} W\right|^{2} \chi^{2}+C|U-\tilde{U}|_{\tilde{p}}^{2}+C|W|_{\tilde{p}}|U-\tilde{U}|_{\tilde{p}}
$$

By (2.42) and (2.46), we get

$$
J_{7} \leq\left(\frac{9}{500}+\frac{6}{1000}\right) \sum_{|\alpha|=\tilde{p}} \int\left|\tilde{\nabla}^{\alpha+1} W\right|^{2} \chi^{2}+C|U-\tilde{U}|_{\tilde{p}}^{2}+C|W|_{\tilde{p}}|U-\tilde{U}|_{\tilde{p}}
$$

By (2.31), (2.32), (2.47) and (2.30), we get

$$
J_{4} \leq \frac{3}{100} \sum_{|\alpha|=\tilde{p}} \int\left|\tilde{\nabla}^{\alpha+1} W\right|^{2} \chi^{2}+C|U-\tilde{U}|_{\tilde{p}}^{2}+C|W|_{\tilde{p}}|U-\tilde{U}|_{\tilde{p}}
$$

By (2.27), (2.28), (2.29), (2.48), and (2.26), we get

$$
\frac{\partial}{\partial t}\left(\left.W\right|_{\tilde{p}} ^{2}\right) \leq C_{1}|W|_{\tilde{p}}^{2}+C_{2}|U-\tilde{U}|_{\tilde{p}}^{2}
$$

where $C_{1}=C_{1}\left(n, \tilde{p}, G,\|\chi\|_{\tilde{p}},\|\widetilde{R m}\|_{\tilde{p}}, C_{s}\left(\Omega, g_{0}\right)\right)>0$ and $C_{2}$ depends on the same parameter as $C_{1}$.

By Gronwall's inequality and (2.49), noting $|W(x, 0)|_{\tilde{p}}=0$, we get

$$
|W|_{\tilde{p}}^{2}(t) \leq e^{C_{1} t} \cdot \int_{0}^{t} C_{2}|U-\tilde{U}|_{\tilde{p}}^{2}(s) d s \leq C_{2} t e^{C_{1} t}|U-\tilde{U}|_{t, \tilde{p}}^{2} .
$$

We can choose $t_{6}=t_{6}\left(\delta, C_{1}, C_{2}\right)=t_{6}\left(n, \tilde{p}, G,\|\chi\|_{\tilde{p}},\|\widetilde{R m}\|_{\tilde{p}}, C_{s}\left(\Omega, g_{0}\right), \delta\right)>0$, such that $C_{2} \cdot t_{6} \cdot e^{C_{1} t_{6}}=\delta^{2}<1$. Then we choose

$$
t_{7}=\min \left\{t_{3}, t_{6}\right\}=t_{7}\left(n, \tilde{p}, G,\|\chi\|_{\tilde{p}+2},\|\widetilde{R m}\|_{\tilde{p}+2}, C_{s}\left(\Omega, g_{0}\right), \delta\right) .
$$

If $t \in\left[0, t_{7}\right]$, we get $|W|_{\tilde{p}}^{2}(t) \leq \delta^{2}|U-\tilde{U}|_{t_{7}, \tilde{p}}^{2}$. Hence

$$
|W|_{t_{7}, \tilde{p}} \leq \delta|U-\tilde{U}|_{t_{7}, \tilde{p}}
$$

That is,

$$
\left|\Phi_{\epsilon}(U)-\Phi_{\epsilon}(\tilde{U})\right|_{t_{7}, \tilde{p}} \leq \delta|U-\tilde{U}|_{t_{7}, \tilde{p}}
$$

Theorem 2.9. There exists $T=T\left(n, \tilde{p}, G,\|\chi\|_{2 \tilde{p}+2},\|\widetilde{R m}\|_{2 \tilde{p}+2}, C_{s}\left(\Omega, g_{0}\right)\right)>0$, such that on $\Omega \times[0, T]$, the degenerate parabolic system (2.3) has a smooth solution.

Proof. Choose $m=2 \tilde{p}+2$ in Proposition 2.7 and $\delta=\frac{1}{2}$ in Proposition 2.8. Then we choose

$$
T=\min \left\{t_{5}, t_{7}\right\}=T\left(n, \tilde{p}, G,\|\chi\|_{2 \tilde{p}+2},\|\widetilde{R m}\|_{2 \tilde{p}+2}, C_{s}\left(\Omega, g_{0}\right)\right) .
$$

Set $U^{(0)}(x, t)=\tilde{g}(x)$ and $U_{\epsilon}^{(i+1)}=\Phi_{\epsilon}\left(U^{(i)}\right)$, where $U_{\epsilon}^{(i)}$ are $(2,0)$-tensors on $\Omega \times[0, T], i=0,1,2 \cdots$. Note that $U^{(0)} \in \mathfrak{A}_{T} \cap \mathbb{B}_{m}(G)_{T}$ for any $m \geq \tilde{p}+2$. By Proposition 2.7, we get $U_{\epsilon}^{(i)} \in \mathfrak{A}_{T} \cap \mathbb{B}_{2 \tilde{p}+2}(G)_{T}$ for any $i$. Then we have the following:

$$
\sup _{(x, t) \in \Omega \times[0, T]} \sup _{|\alpha| \leq \tilde{p}+2}\left|\tilde{\nabla}^{\alpha} U_{\epsilon}^{(i)}(x, t)\right| \leq C_{1}\left|U_{\epsilon}^{(i)}\right|_{T, 2 \tilde{p}+2} \leq C_{1} G
$$

for any $i \geq 0$, where $C_{1}=C_{1}\left(C_{s}\left(\Omega, g_{0}\right), \tilde{p}\right)$, and note that $C_{1}$ is independent of $i$. 
By (2.4), we get

$$
\sup _{|\beta| \leq \frac{\tilde{p}+2}{2}} \sup _{(x, t) \in \Omega \times[0, T]}\left|D_{t}^{\beta} U_{\epsilon}^{(i)}(x, t)\right| \leq C_{2}
$$

for any $i \geq 0$, where $C_{2}=C_{2}\left(C_{s}\left(\Omega, g_{0}\right), n, \tilde{p}, G,\|\chi\|_{2},\|\widetilde{R m}\|_{0}\right)$. Note that $C_{2}$ is independent of $i$.

Since $\left\{U_{\epsilon}^{(i)}\right\}_{i=1}^{\infty}$ are uniformly bounded in $\mathbb{B}_{2 \tilde{p}+2}(G)_{T}$, we can choose the subsequence $U_{\epsilon}^{\left(k_{i}\right)} \rightarrow U_{\epsilon}^{\infty}$ in $C^{\tilde{p}+2, \frac{\tilde{p}+2}{2}}(\Omega \times[0, T])$ by the Rellich-Kondrachov Compactness theorem, and $U_{\epsilon}^{\infty} \in \mathbb{B}_{\tilde{p}+2}(G)_{T}$. That is why we chose $m=2 \tilde{p}+2$ in Proposition 2.7 at the beginning of the proof. For $k_{i} \geq m>0$, we get

$$
\begin{aligned}
\left|U_{\epsilon}^{(m)}-U_{\epsilon}^{\left(k_{i}\right)}\right|_{T, \tilde{p}}= & \left|\Phi_{\epsilon}^{m}\left(U^{(0)}\right)-\Phi_{\epsilon}^{k_{i}}\left(U^{(0)}\right)\right|_{T, \tilde{p}} \leq \delta^{m}\left|U^{(0)}-\Phi_{\epsilon}^{k_{i}-m}\left(U^{(0)}\right)\right|_{T, \tilde{p}} \\
& \leq 2\left(\frac{1}{2}\right)^{m} G .
\end{aligned}
$$

Hence

$$
\lim _{m \rightarrow \infty}\left|U_{\epsilon}^{(m)}-U_{\epsilon}^{\infty}\right|_{T, \tilde{p}} \leq \lim _{m \rightarrow \infty}\left(\left|U_{\epsilon}^{(m)}-U_{\epsilon}^{\left(k_{i}\right)}\right|_{T, \tilde{p}}+\left|U_{\epsilon}^{\left(k_{i}\right)}-U_{\epsilon}^{\infty}\right|_{T, \tilde{p}}\right)=0 .
$$

Now

$$
\begin{aligned}
\mid U_{\epsilon}^{\infty} & -\left.\Phi_{\epsilon}\left(U_{\epsilon}^{\infty}\right)\right|_{T, \tilde{p}}=\lim _{m \rightarrow \infty}\left|U_{\epsilon}^{(m)}-\Phi_{\epsilon}\left(U_{\epsilon}^{\infty}\right)\right|_{T, \tilde{p}} \\
& =\lim _{m \rightarrow \infty}\left|\Phi_{\epsilon}\left(U_{\epsilon}^{(m-1)}\right)-\Phi_{\epsilon}\left(U_{\epsilon}^{\infty}\right)\right|_{T, \tilde{p}} \leq \frac{1}{2} \lim _{m \rightarrow \infty}\left|U_{\epsilon}^{(m-1)}-U_{\epsilon}^{\infty}\right|_{T, \tilde{p}}=0 .
\end{aligned}
$$

Then we have

$$
\left|U_{\epsilon}^{\infty}-\Phi_{\epsilon}\left(U_{\epsilon}^{\infty}\right)\right|_{C^{0}} \leq C_{s}\left(\Omega, g_{0}\right)\left|U_{\epsilon}^{\infty}-\Phi_{\epsilon}\left(U_{\epsilon}^{\infty}\right)\right|_{T, \tilde{p}}=0,
$$

and hence

$$
U_{\epsilon}^{\infty}=\Phi_{\epsilon}\left(U_{\epsilon}^{\infty}\right) .
$$

By (2.52) and (2.53), we get $\left\{U_{\epsilon}^{\infty}\right\}_{0<\epsilon \leq 1}$ is uniformly bounded in $C^{\tilde{p}+2, \frac{\tilde{p}+2}{2}}(\Omega \times$ $[0, T])$. So there exists a subsequence $\left\{U_{\epsilon_{i}}^{\infty}\right\}_{i=1}^{\infty}$, such that

$$
\lim _{i \rightarrow \infty} U_{\epsilon_{i}}^{\infty}=\hat{U}^{\infty} \quad \text { in } \quad C^{\tilde{p}, \frac{\tilde{p}}{2}}(\Omega \times[0, T]),
$$

where $\lim _{i \rightarrow \infty} \epsilon_{i}=0$.

On the other hand, by (2.54) and (2.4) we have

$$
\left(U_{\epsilon_{i}}^{\infty}\right)_{t}=\left(\chi^{2}+\epsilon_{i}\right)\left(U_{\epsilon_{i}}^{\infty}\right)^{\alpha \beta} \tilde{\nabla}_{\alpha} \tilde{\nabla}_{\beta}\left(U_{\epsilon_{i}}^{\infty}\right)+D\left(U_{\epsilon_{i}}^{\infty}\right)+B\left(U_{\epsilon_{i}}^{\infty}, \tilde{\nabla} U_{\epsilon_{i}}^{\infty}\right) .
$$

Let $i \rightarrow \infty$ in 2.55); we get

$$
\left(\hat{U}^{\infty}\right)_{t}=\chi^{2}\left(\hat{U}^{\infty}\right)^{\alpha \beta} \tilde{\nabla}_{\alpha} \tilde{\nabla}_{\beta}\left(\hat{U}^{\infty}\right)+D\left(\hat{U}^{\infty}\right)+B\left(\hat{U}^{\infty}, \tilde{\nabla} \hat{U}^{\infty}\right) .
$$

Then $\hat{U}^{\infty}$ is a solution of (2.3) on $\Omega \times[0, T]$, and $\hat{U}^{\infty} \in C^{\tilde{p}}, \frac{\tilde{p}}{2}(\Omega \times[0, T])$. By regularity theory of the parabolic system (note that we have local regularity property where $\chi(x)>0$, and $\hat{U}^{\infty}(x, t)=\tilde{g}_{i j}(x)$ where $\left.\chi(x)=0\right)$, we have

$$
\hat{U}^{\infty} \in C^{\infty}(\Omega \times[0, T]) .
$$

Hence $\hat{U}^{\infty}$ is a smooth solution of (2.3) on $\Omega \times[0, T]$. 


\section{Evolution Equations of CURVAture Under the LOCAL RicCi Flow}

Under local Ricci flow, the behavior of curvature tensors are affected by their evolution equations. These equations have simpler versions in Ricci flow context by just taking the cut-off function $\chi(x) \equiv 1$.

Now we calculate the evolution equation of the curvature tensor $R m$. We use the following notation: $R_{i j k}^{l}=g^{l p} R_{i j k p}, R_{i}^{r}=g^{r p} R_{i p}$, and $R$ is the scalar curvature.

Lemma 3.1. Under the local Ricci flow (1.2),

$$
\frac{\partial}{\partial t} R_{i j k l}=\chi^{2} \Delta R_{i j k l}+Q+I_{1}+I_{2}+I_{3}
$$

where $Q, I_{1}, I_{2}, I_{3}$ are defined in (3.8), (3.4), (3.5), (3.6).

Proof. First, if $\frac{\partial}{\partial t} g_{i j}=h_{i j}$, then (see [1])

$$
\frac{\partial}{\partial t} R_{i j k}^{l}=\frac{1}{2} g^{l p}\left[\nabla_{i} \nabla_{k} h_{j p}+\nabla_{j} \nabla_{p} h_{i k}-\nabla_{i} \nabla_{p} h_{j k}-\nabla_{j} \nabla_{k} h_{i p}-R_{i j k}^{q} h_{q p}-R_{i j p}^{q} h_{k q}\right] .
$$

In the local Ricci flow case,

$$
h_{i j}=-2 \chi^{2} R_{i j}
$$

Now

$$
\frac{\partial}{\partial t} R_{i j k l}=\left(-2 \chi^{2} R_{l m}\right) R_{i j k}^{m}+g_{l m}\left(\frac{\partial}{\partial t} R_{i j k}^{m}\right) .
$$

We calculate $g_{l m} \frac{\partial}{\partial t} R_{i j k}^{m}$ first:

$$
g_{l m} \frac{\partial}{\partial t} R_{i j k}^{m}=\frac{1}{2}\left[\nabla_{i} \nabla_{k} h_{j l}+\nabla_{j} \nabla_{l} h_{i k}-\nabla_{i} \nabla_{l} h_{j k}-\nabla_{j} \nabla_{k} h_{i l}-R_{i j k}^{q} h_{q l}-R_{i j l}^{q} h_{k q}\right] .
$$

We calculate $\nabla_{i} \nabla_{k} h_{j l}$ as a sample, using the notation $\chi_{i}=\nabla_{i} \chi$ and $\chi_{i j}=$ $\nabla_{i} \nabla_{j} \chi$ :

$$
\nabla_{i} \nabla_{k} h_{j l}=-2\left(\chi^{2} \nabla_{i} \nabla_{k} R_{j l}+2 \chi \chi_{i} \nabla_{k} R_{j l}+2 \chi \chi_{k} \nabla_{i} R_{j l}+2 \chi_{i} \chi_{k} R_{j l}+2 \chi \chi_{i k} R_{j l}\right) .
$$

A straightforward computation gives

$$
\begin{aligned}
g_{l m} \frac{\partial}{\partial t} R_{i j k}^{m}= & \chi^{2}\left(\nabla_{i} \nabla_{l} R_{j k}+\nabla_{j} \nabla_{k} R_{i l}-\nabla_{i} \nabla_{k} R_{j l}-\nabla_{j} \nabla_{l} R_{i k}\right) \\
& +\chi^{2}\left(R_{i j k}^{q} R_{q l}+R_{i j l}^{q} R_{k q}\right)+I_{1}+I_{2}+I_{3},
\end{aligned}
$$

where

$$
\begin{aligned}
I_{1} & =2 \chi\left[\left(\chi_{k} \nabla_{j} R_{i l}+\chi_{j} \nabla_{k} R_{i l}+\chi_{l} \nabla_{i} R_{j k}\right.\right. \\
& \left.\left.+\chi_{i} \nabla_{l} R_{j k}\right)-\left(\chi_{k} \nabla_{i} R_{j l}+\chi_{i} \nabla_{k} R_{j l}+\chi_{l} \nabla_{j} R_{i k}+\chi_{j} \nabla_{l} R_{i k}\right)\right], \\
I_{2} & =2\left[\left(\chi_{i} \chi_{l} R_{j k}+\chi_{j} \chi_{k} R_{i l}\right)-\left(\chi_{i} \chi_{k} R_{j l}+\chi_{j} \chi_{l} R_{i k}\right)\right], \\
I_{3} & =2 \chi\left[\left(\chi_{i l} R_{j k}+\chi_{j k} R_{i l}\right)-\left(\chi_{i k} R_{j l}+\chi_{j l} R_{i k}\right)\right] .
\end{aligned}
$$

Now we compute $\Delta R_{i j k l}$. From the formula for $\Delta R_{i j k}^{l}$ in [1],

$$
\begin{aligned}
& \Delta R_{i j k l}=\Delta\left(g_{l m} R_{i j k}^{m}\right)=g_{l m} \Delta R_{i j k}^{m} \\
& =\left(\nabla_{i} \nabla_{l} R_{j k}+\nabla_{j} \nabla_{k} R_{i l}-\nabla_{i} \nabla_{k} R_{j l}-\nabla_{j} \nabla_{l} R_{i k}\right) \\
& \quad+\left(R_{j}^{r} R_{i r k l}-R_{i}^{r} R_{j r k l}\right)-g^{p q}\left(R_{i j p}^{r} R_{r q k l}-2 R_{p i k}^{r} R_{j q r l}+2 R_{j q k}^{r} R_{p i r l}\right) .
\end{aligned}
$$

By (3.2), (3.3) and (3.7),

$$
\frac{\partial}{\partial t} R_{i j k l}=\chi^{2} \Delta R_{i j k l}+Q+I_{1}+I_{2}+I_{3}
$$


where

$$
\begin{aligned}
Q & =Q_{1}+Q_{2}, \\
Q_{1} & =\chi^{2} g^{p q}\left(R_{i j p}^{r} R_{r q k l}-2 R_{p i k}^{r} R_{j q r l}+2 R_{j q k}^{r} R_{p i r l}\right), \\
Q_{2} & =-\chi^{2}\left(R_{m j k l} R_{i}^{m}+R_{i m k l} R_{j}^{m}+R_{i j m l} R_{k}^{m}+R_{i j k m} R_{l}^{m}\right) .
\end{aligned}
$$

For our applications, we need to estimate $\frac{\partial}{\partial t}|R m|$.

Lemma 3.2. Under the local Ricci flow (1.2), we have

$$
\begin{aligned}
\frac{\partial}{\partial t}|R m| & \leq \chi^{2} \Delta|R m|+\left(\frac{\chi^{2}|\nabla| R m||^{2}}{|R m|}\right)-(1-\epsilon)\left(\frac{\chi^{2}|\nabla R m|^{2}}{|R m|}\right) \\
& +10 \chi^{2}|R m|^{2}+\left(\frac{10^{5}}{\epsilon}\right) n|\nabla \chi|^{2}|R m|+\left(\frac{8 \chi g^{r i} g^{s j} g^{p k} g^{q l} R_{r s p q} \chi_{i l} R_{j k}}{|R m|}\right),
\end{aligned}
$$

where $\epsilon$ is any positive constant satisfying $0<\epsilon<1$.

Proof.

$$
\frac{\partial}{\partial t}|R m|^{2}=2 g^{r i} g^{s j} g^{p k} g^{q l} R_{r s p q}\left(\frac{\partial}{\partial t} R_{i j k l}\right)+\frac{\partial}{\partial t}\left(g^{r i} g^{s j} g^{p k} g^{q l}\right) R_{r s p q} R_{i j k l} .
$$

Note that

$$
\frac{\partial}{\partial t}\left(g^{r i}\right) g^{s j} g^{p k} g^{q l} R_{r s p q} R_{i j k l}=2 \chi^{2} g^{r i} g^{s j} g^{p k} g^{q l} R_{r s p q} R_{m j k l} R_{i}^{m} .
$$

Similarly,

$$
\frac{\partial}{\partial t}\left(g^{r i} g^{s j} g^{p k} g^{q l}\right) R_{r s p q} R_{i j k l}=-2 g^{r i} g^{s j} g^{p k} g^{q l} R_{r s p q} Q_{2}
$$

Hence

$$
\frac{\partial}{\partial t}|R m|^{2}=\chi^{2}\left[\Delta\left(|R m|^{2}\right)-2|\nabla R m|^{2}\right]+2 g^{r i} g^{s j} g^{p k} g^{q l} R_{r s p q}\left[Q_{1}+I_{1}+I_{2}+I_{3}\right] .
$$

Letting $f=|R m|$,

$$
\frac{\partial}{\partial t}\left(f^{2}\right)=\chi^{2} \Delta\left(f^{2}\right)-2 \chi^{2}|\nabla R m|^{2}+2 g^{r i} g^{s j} g^{p k} g^{q l} R_{r s p q}\left[Q_{1}+I_{1}+I_{2}+I_{3}\right] .
$$

First we have the following estimate:

$$
2 g^{r i} g^{s j} g^{p k} g^{q l} R_{r s p q} Q_{1} \leq 10 \chi^{2} f^{3} .
$$

It is easy to get

$$
\begin{gathered}
2 g^{r i} g^{s j} g^{p k} g^{q l} R_{r s p q} I_{1} \leq 32 \sqrt{n} \chi|\nabla \chi||\nabla R m| f \leq 2 \epsilon(\chi|\nabla R m|)^{2}+\left(\frac{10^{4}}{\epsilon}\right) n|\nabla \chi|^{2} f^{2}, \\
2 g^{r i} g^{s j} g^{p k} g^{q l} R_{r s p q} I_{2} \leq 16 \sqrt{n}|\nabla \chi|^{2} f^{2} .
\end{gathered}
$$

We can use the normal coordinate system to simplify:

$$
2 g^{r i} g^{s j} g^{p k} g^{q l} R_{r s p q} I_{3}=16 \chi \sum_{i j k l}\left(\chi_{i l} R_{j k} R_{i j k l}\right)=16 \chi g^{r i} g^{s j} g^{p k} g^{q l} R_{r s p q} \chi_{i l} R_{j k} \text {. }
$$

Using normal coordinates it is also easy to see

$$
|\nabla| R m|| \leq|\nabla R m| .
$$

Then

$$
\begin{aligned}
\frac{\partial}{\partial t}\left(f^{2}\right) \leq & 2 f \chi^{2} \Delta f+2 \chi^{2}|\nabla f|^{2}-(2-2 \epsilon) \chi^{2}|\nabla R m|^{2}+10 \chi^{2} f^{3} \\
& +\left(\frac{10^{5}}{\epsilon}\right) n|\nabla \chi|^{2} f^{2}+16 \chi g^{r i} g^{s j} g^{p k} g^{q l} R_{r s p q} \chi_{i l} R_{j k} .
\end{aligned}
$$


Finally,

$$
\begin{aligned}
\frac{\partial}{\partial t} f \leq & \chi^{2} \Delta f+\left(\frac{\chi^{2}|\nabla f|^{2}}{f}\right)-(1-\epsilon)\left(\frac{\chi^{2}|\nabla R m|^{2}}{f}\right) \\
& +10 \chi^{2} f^{2}+\left(\frac{10^{5}}{\epsilon}\right) n|\nabla \chi|^{2} f+\left(\frac{8 \chi g^{r i} g^{s j} g^{p k} g^{q l} R_{r s p q} \chi_{i l} R_{j k}}{f}\right) .
\end{aligned}
$$

Replacing $f$ with $|R m|$, we get our conclusion.

Using the formula in Chapter 3 of [1, we can get the following formula (because the calculation is very similar to the reduction of Lemma 3.1 we omit the calculation here, and just give the formula):

Lemma 3.3. Under the local Ricci flow (1.2),

$$
\begin{aligned}
\frac{\partial}{\partial t} R_{i j} & =\chi^{2} \Delta\left(R_{i j}\right)+J_{1}+J_{2}+J_{3}+J_{4}, \\
\text { where } J_{1} & =2 \chi g^{p q} \chi_{p} \nabla_{q} R_{i j}+\chi\left(\chi_{i} \nabla_{j} R+\chi_{j} \nabla_{i} R\right) \\
& -2 \chi \chi_{p} g^{p q}\left(\nabla_{i} R_{q j}+\nabla_{j} R_{q i}\right), \\
J_{2} & =-2 g^{p q} \chi\left(\chi_{i p} R_{q j}+\chi_{j p} R_{q i}\right)+2 \chi_{i j} R, \\
J_{3} & =-2 g^{p q}\left(\chi_{i} \chi_{p} R_{q j}+\chi_{j} \chi_{p} R_{q i}\right)+2 \chi_{i} \chi_{j} R, \\
J_{4} & =2 \chi^{2} g^{p q}\left(R_{q i j}^{r} R_{r p}-R_{i p} R_{q j}\right) .
\end{aligned}
$$

Imitating the reduction of Lemma 3.2, we can also get the following theorem about $|R c|$ :

Lemma 3.4. Under the local Ricci flow (1.2),

$$
\begin{aligned}
\frac{\partial}{\partial t}|R c| & \leq \chi^{2} \Delta|R c|+\left(\frac{\chi^{2}|\nabla| R c||^{2}}{|R c|}\right)-(1-\epsilon)\left(\frac{\chi^{2}|\nabla R c|^{2}}{|R c|}\right) \\
& +\left(\frac{10^{5}}{\epsilon}\right) n|\nabla \chi|^{2}|R c|+(2+\sqrt{n}) \chi^{2}|R c||R m|+\left(\frac{g^{i k} g^{j l} R_{k l} J_{2}}{|R c|}\right)
\end{aligned}
$$

where $J_{2}$ in the last line comes from (3.13), and $\epsilon$ is any positive constant satisfying $0<\epsilon<1$.

Proof. Similar to the proof of Lemma 3.2 .

If $T_{1}$ and $T_{2}$ are two tensors, we use $T_{1} * T_{2}$ to denote all possible tensor products between them.

\section{Lemma 3.5.}

$$
\begin{gathered}
\frac{\partial}{\partial t}\left(\nabla^{m} R m\right)=\chi^{2} \Delta\left(\nabla^{m} R m\right)+\sum_{i+j+k=m+2, k \leq m+1} \nabla^{i} \chi * \nabla^{j} \chi * \nabla^{k} R m \\
+\sum_{i+j+k=m} \nabla^{i}\left(\chi^{2}\right) * \nabla^{j} R m * \nabla^{k} R m .
\end{gathered}
$$


Proof. By induction, when $m=0$, it is true from Lemma 3.1. Suppose the conclusion holds for $m-1$. Then we have

$$
\begin{aligned}
\frac{\partial}{\partial t}\left(\nabla \nabla^{m-1} R m\right) & =\nabla^{m-1} R m * \nabla\left(\chi^{2} R c\right)+\nabla\left(\chi^{2} \Delta \nabla^{m-1} R m\right) \\
& +\nabla\left[\sum_{i+j+k=m+1, k \leq m} \nabla^{i} \chi * \nabla^{j} \chi * \nabla^{k} R m\right] \\
& +\nabla\left[\sum_{i+j+k=m-1} \nabla^{i}\left(\chi^{2}\right) * \nabla^{j} R m * \nabla^{k} R m\right]
\end{aligned}
$$

and

$$
\begin{aligned}
\nabla\left[\chi^{2} \Delta \nabla^{m-1} R m\right]= & \chi^{2} \Delta \nabla^{m} R m+\chi^{2} \nabla^{m-1} R m * \nabla R m \\
& +\chi^{2} \nabla^{m} R m * R m+\nabla\left(\chi^{2}\right) * \Delta \nabla^{m-1} R m .
\end{aligned}
$$

We can consider $\nabla^{m-1} R m * \nabla\left(\chi^{2} R c\right), \chi^{2} \nabla^{m-1} R m * \nabla R m, \chi^{2} \nabla^{m} R m * R m$ as the terms in $\nabla\left[\sum_{i+j+k=m-1} \nabla^{i}\left(\chi^{2}\right) * \nabla^{j} R m * \nabla^{k} R m\right]$, and $\nabla\left(\chi^{2}\right) * \Delta \nabla^{m-1} R m$ as one of the terms in $\nabla\left[\sum_{i+j+k=m+1, k \leq m} \nabla^{i} \chi * \nabla^{j} \chi * \nabla^{k} R m\right]$. Then we get our equation.

Similarly, we have

\section{Lemma 3.6.}

$$
\begin{aligned}
\frac{\partial}{\partial t} \nabla^{m}\left(\chi^{2} R m\right) & =\chi^{2} \Delta \nabla^{m}\left(\chi^{2} R m\right) \\
& +\sum_{i+j+k=m+2, k \leq m+1} \nabla^{i} \chi * \nabla^{j} \chi * \nabla^{k}\left(\chi^{2} R m\right) \\
& +\sum_{i+j+k=m, k \leq m-1} \nabla^{i}\left(\chi^{2}\right) * \nabla^{j}\left(\chi^{2} R m\right) * \nabla^{k} R m+\chi^{2} R m * \nabla^{m}\left(\chi^{2} R m\right)
\end{aligned}
$$

and

$$
\frac{\partial}{\partial t}\left|\nabla^{m} \chi\right| \leq C(n, k) \sum_{k \leq m-1}\left|\nabla^{k}\left(\chi^{2} R c\right)\right| \cdot\left|\nabla^{m-k} \chi\right| .
$$

\section{EXTENSION OF THE LOCAL RiCCI FLOW}

First we prove the following ODE comparison lemmas, which will be used in the later proofs of this section.

Lemma 4.1. Assume $f(t)$ and $g(t)$ are nonnegative functions, $t \in[0, T), b$ is a nonnegative constant, and

$$
\frac{d f}{d t} \leq g+b f
$$

Then on $[0, T)$ :

$$
f(t) \leq\left[f(0)+\int_{0}^{t} e^{-b s} g(s) d s\right] e^{b t}
$$

Proof. Let $h(t)=\left[f(0)+\int_{0}^{t} e^{-b s} g(s) d s\right] e^{b t}$. Then $h(0)=f(0)$, and $h^{\prime}(t) \geq f^{\prime}(t)$ by assumption. Hence $h(t) \geq f(t)$. 
Lemma 4.2. Assume $f(t)$ is a nonnegative function, $t \in[0, T), a, b, c$ are nonnegative constants, and

$$
\frac{d f}{d t} \leq a \int_{0}^{t} f(s) d s+b f(t)+c .
$$

Then there exist positive constants $w, k$ which depend on $a, b, c$ and $f(0)$ such that

$$
f(t) \leq w e^{k t}, \quad t \in[0, T) .
$$

Proof. It suffices to choose $w, k$ large enough to ensure

$$
\left(w e^{k t}\right)^{\prime}>a\left[\int_{0}^{t}\left(w e^{k s}\right) d s\right]+b\left(w e^{k t}\right)+c \quad \text { and } \quad w>f(0)
$$

to get the comparison function.

We define $|\nabla \chi|_{(g(t), \infty)}=\max _{x \in \Omega}|\nabla \chi(x)|_{g(t)}$. Recall that we have defined $C_{s}(\Omega, g(t))$ in (2.6). For simplicity we use the notation $C_{s}(\Omega, t)$ to replace it.

Lemma 4.3. If $\left|\chi^{2} R m\right|_{(g(t), \infty)}$ is uniformly bounded along a given solution $g(t)$ of local Ricci flow, $t \in[0, T), 0<T<+\infty$, then $|\nabla \chi|_{(g(t), \infty)}$ and the Sobolev constant $C_{s}(\Omega, t)$ are uniformly bounded on $[0, T)$.

Proof. Because $\Omega$ is a bounded domain in $\left(M, g_{0}\right)$, by the proof of Theorem 3.5 in [6] we get that $C_{s}(\Omega, 0)$ is finite. Then one only needs to notice that the metrics $\{g(t)\}_{t \in[0, T)}$ are uniformly equivalent to each other by assumption.

Lemma 4.4. Assume $g(t), t \in[0, T), 0<T<+\infty$, is a smooth solution to local Ricci flow on $\left(M^{n}, g_{0}\right)$, and $\left|\chi^{2} R m\right|_{(g(t), \infty)}$ is uniformly bounded on $[0, T)$. Then for any $p>100,|R m|_{\left(g(t), L^{p}(\Omega)\right)} \triangleq \int_{\Omega}|R m|_{g(t)}^{p} d V_{g(t)}$ is uniformly bounded on $[0, T)$.

Proof. Using Lemma 3.1 ,

$$
\frac{\partial}{\partial t}\left(\int_{\Omega}|R m|_{g(t)}^{p} d V_{g(t)}\right)=p\left(I_{1}+I_{2}+I_{3}+I_{4}+I_{5}\right)
$$

where

$$
\begin{gathered}
I_{1}=-\int_{\Omega} \chi^{2}|\nabla R m|^{2}|R m|^{p-2}, \quad I_{2}=\int_{\Omega}|R m|^{p-2}\langle\chi \nabla \chi * \nabla R m, R m\rangle, \\
I_{3}=\int_{\Omega}|R m|^{p-2}\left\langle\chi^{2} R m * R m, R m\right\rangle, \quad I_{4}=\int_{\Omega}|R m|^{p-2}\langle\nabla \chi * \nabla \chi * R m, R m\rangle, \\
I_{5}=\int_{\Omega}|R m|^{p-2}\left\langle\chi \nabla^{2} \chi * R m, R m\right\rangle
\end{gathered}
$$

and

$$
\begin{gathered}
I_{2} \leq C \int_{\Omega}|R m|^{p}+\frac{1}{16} \int_{\Omega} \chi^{2}|\nabla R m|^{2}|R m|^{p-2}, \\
I_{3} \leq \max _{t \in[0, T)}\left|\chi^{2} R m\right|_{(g(t), \infty)} \cdot \int_{\Omega}|R m|^{p} \leq C \int_{\Omega}|R m|^{p}, \\
I_{4} \leq C \max _{t \in[0, T)}|\nabla \chi|_{(g(t), \infty)} \int_{\Omega}|R m|^{p} \leq C \int_{\Omega}|R m|^{p} .
\end{gathered}
$$

Applying integration by parts and Lemma 4.3 yields

$$
I_{5}=\int_{\Omega}|R m|^{p-2}\left\langle\chi \nabla^{2} \chi * R m, R m\right\rangle \leq C \int_{\Omega}|R m|^{p}+\frac{1}{16} \int_{\Omega} \chi^{2}|\nabla R m|^{2}|R m|^{p-2} .
$$


Then

$$
\frac{\partial}{\partial t} \int_{\Omega}|R m|^{p} \leq C \int_{\Omega}|R m|^{p}
$$

We get

$$
\int_{\Omega}|R m|_{g(t)}^{p} d V_{g(t)} \leq e^{C T} \int_{\Omega}|R m|_{g_{0}}^{p} d V_{g_{0}}
$$

Hence $|R m|_{\left(g(t), L^{p}(\Omega)\right)}$ are uniformly bounded on $[0, T)$.

Proposition 4.5. If $g(t)$ is a smooth solution of the local Ricci flow on $\left(M^{n}, g_{0}\right)$, $t \in[0, T), 0<T<+\infty$ and $m$ is a nonnegative integer. Assume on $[0, T)$, for any $q>100$, that $\left|\nabla^{i} \chi\right|_{\left(g(t), L^{q}(\Omega)\right)}$ are uniformly bounded, where $0 \leq i \leq m+1$, and $\left|\chi^{2} R m\right|_{(g(t), \infty)}$ are uniformly bounded. Then for any $p>100,\left|\nabla^{m} R m\right|_{\left(g(t), L^{p}(\Omega)\right)}$ is uniformly bounded on $[0, T)$.

Proof. By induction. When $m=0$, the conclusion follows from Lemma 4.4, For $m \geq 1$, assume that for any $q>100, \int_{\Omega}\left|\nabla^{k} R m\right|_{g(t)}^{q} d V_{g(t)}$ is uniformly bounded on $[0, T)$, where $0 \leq k \leq m-1$. For simplicity, we use $\int$ instead of $\int_{\Omega}$ in the rest of the proof.

Using Lemma 3.5

$$
\frac{\partial}{\partial t} \int\left|\nabla^{m} R m\right|_{g(t)}^{p} d V_{g(t)} \leq p\left(I_{1}+I_{2}+I_{3}\right)+C \int\left|\nabla^{m} R m\right|_{g(t)}^{p} d V_{g(t)},
$$

where

$$
\begin{gathered}
I_{1}=\int\left|\nabla^{m} R m\right|^{p-2}\left\langle\chi^{2} \Delta \nabla^{m} R m, \nabla^{m} R m\right\rangle, \\
I_{2}=\int\left|\nabla^{m} R m\right|^{p-2} \sum_{i+j+k=m+2, k \leq m+1}\left\langle\nabla^{i} \chi * \nabla^{j} \chi * \nabla^{k} R m, \nabla^{m} R m\right\rangle, \\
I_{3}=\int\left|\nabla^{m} R m\right|^{p-2} \sum_{i+j+k=m}\left\langle\nabla^{i}\left(\chi^{2}\right) * \nabla^{j}(R m) * \nabla^{k} R m, \nabla^{m} R m\right\rangle .
\end{gathered}
$$

Using integration by parts and the Hölder inequality,

$$
\begin{aligned}
I_{2} \leq & C \int\left|\nabla^{m} R m\right|^{p-1} \cdot\left|\nabla^{m+1} R m\right| \cdot|\nabla \chi| \cdot|\chi|+C \int\left|\nabla^{m} R m\right|^{p} \cdot|\nabla \chi|^{2} \\
+ & C \sum_{i+j+k=m+2,1 \leq k \leq m-1}\left[\left(\int\left|\nabla^{m} R m\right|^{p}\right)^{\frac{p-1}{p}} \cdot\left(\int\left|\nabla^{k} R m\right|^{3 p}\right)^{\frac{1}{3 p}}\right. \\
& \left.\cdot\left(\int\left|\nabla^{i} \chi\right|^{3 p}\right)^{\frac{1}{3 p}} \cdot\left(\int\left|\nabla^{j} \chi\right|^{3 p}\right)^{\frac{1}{3 p}}\right]+I_{4} \\
+ & C \sum_{i+j=m+2, i, j \leq m+1}\left[\left(\int\left|\nabla^{m} R m\right|^{p}\right)^{\frac{p-1}{p}} \cdot\left(\int|R m|^{3 p}\right)^{\frac{1}{3 p}}\right. \\
& \left.\cdot\left(\int\left|\nabla^{i} \chi\right|^{3 p}\right)^{\frac{1}{3 p}} \cdot\left(\int\left|\nabla^{j} \chi\right|^{3 p}\right)^{\frac{1}{3 p}}\right] .
\end{aligned}
$$

Using the Schwartz inequality,

$$
\begin{aligned}
I_{2} \leq & \frac{1}{16} \int \chi^{2}\left|\nabla^{m+1} R m\right|^{2}\left|\nabla^{m} R m\right|^{p-2}+C \int\left|\nabla^{m} R m\right|^{p} \\
& +C\left|\nabla^{m} R m\right|_{\left(g(t), L^{p}(\Omega)\right)}^{p-1}+I_{4},
\end{aligned}
$$


where $I_{4} \triangleq C \int\left|\nabla^{m} R m\right|^{p-2} \cdot\left\langle\chi \nabla^{m+2} \chi * R m, \nabla^{m} R m\right\rangle$. Using integration by parts on $I_{4}$,

$$
\begin{aligned}
I_{4} \leq & C \int \chi\left|\nabla^{m} R m\right|^{p-2} \cdot\left|\nabla^{m+1} R m\right| \cdot\left|\nabla^{m+1} \chi\right| \cdot|R m| \\
& +C \int\left|\nabla^{m} R m\right|^{p-1} \cdot\left|\nabla^{m+1} \chi\right| \cdot|\nabla \chi| \cdot|R m|+I_{5} \\
\leq & C\left(\int \chi^{2}\left|\nabla^{m} R m\right|^{p-2}\left|\nabla^{m+1} R m\right|^{2}\right)^{\frac{1}{2}} \cdot\left(\int\left|\nabla^{m} R m\right|^{p}\right)^{\frac{p-1}{2 p}} \\
& +C\left|\nabla^{m} R m\right|_{\left(g(t), L^{p}(\Omega)\right)}^{p-1}+I_{5} .
\end{aligned}
$$

In the last inequality, we used the assumption of the proposition and Lemma 4.4. Then

$$
I_{4} \leq \frac{1}{16} \int \chi^{2}\left|\nabla^{m} R m\right|^{p-2}\left|\nabla^{m+1} R m\right|^{2}+C\left|\nabla^{m} R m\right|_{\left(g(t), L^{p}(\Omega)\right)}^{p-1}+I_{5},
$$

where $\left.I_{5} \triangleq C\left|\int\right| \nabla^{m} R m\right|^{p-2}\left\langle\chi \nabla^{m+1} \chi * \nabla R m, \nabla^{m} R m\right\rangle \mid$.

To estimate $I_{5}$, there are two cases:

If $m=1$, then $\nabla R m=\nabla^{m} R m$, and by definition of $I_{5}$,

$$
\begin{aligned}
I_{5} & \leq C \int\left|\nabla^{m} R m\right|^{p-1}\left|\nabla^{m+1} R m\right| \cdot\left|\nabla^{m} \chi\right| \cdot|\chi|+C \int\left|\nabla^{m} R m\right|^{p} \cdot|\nabla \chi|^{2} \\
& \leq \frac{1}{16} \int \chi^{2}\left|\nabla^{m} R m\right|^{p-2}\left|\nabla^{m+1} R m\right|^{2}+C \int\left|\nabla^{m} R m\right|^{p}\left[\left|\nabla^{m} \chi\right|^{2}+|\nabla \chi|^{2}\right] \\
& \leq \frac{1}{16} \int \chi^{2}\left|\nabla^{m} R m\right|^{p-2}\left|\nabla^{m+1} R m\right|^{2}+C \int\left|\nabla^{m} R m\right|^{p} .
\end{aligned}
$$

The last inequality above uses the fact that $m=1$ and $|\nabla \chi|_{(g(t), \infty)}$ are uniformly bounded on $[0, T)$ :

If $m \geq 2$, by induction assumption, for any $q>100, \int|\nabla R m|^{q}$ is uniformly bounded on $[0, T)$ :

$$
\begin{gathered}
I_{5} \leq C \int\left|\nabla^{m} R m\right|^{p-1} \cdot\left|\nabla^{m+1} \chi\right| \cdot|\nabla R m| \\
\leq C\left(\int\left|\nabla^{m} R m\right|^{p}\right)^{\frac{p-1}{p}} \cdot\left(\int\left|\nabla^{m+1} \chi\right|^{2 p}\right)^{\frac{1}{2 p}} \cdot\left(\int|\nabla R m|^{2 p}\right)^{\frac{1}{2 p}}, \\
I_{5} \leq C\left|\nabla^{m} R m\right|_{\left(g(t), L^{p}(\Omega)\right)}^{p-1} .
\end{gathered}
$$

By (4.4) and (4.5),

$$
I_{5} \leq \frac{1}{16} \int \chi^{2}\left|\nabla^{m} R m\right|^{p-2}\left|\nabla^{m+1} R m\right|^{2}+C \int\left|\nabla^{m} R m\right|^{p}+C\left|\nabla^{m} R m\right|_{\left(g(t), L^{p}(\Omega)\right)}^{p-1} .
$$

By (4.3) and (4.6),

$$
I_{4} \leq \frac{1}{8} \int \chi^{2}\left|\nabla^{m} R m\right|^{p-2}\left|\nabla^{m+1} R m\right|^{2}+C \int\left|\nabla^{m} R m\right|^{p}+C\left|\nabla^{m} R m\right|_{\left(g(t), L^{p}(\Omega)\right)}^{p-1} .
$$

By (4.2) and (4.7),

$$
I_{2} \leq \frac{3}{16} \int \chi^{2}\left|\nabla^{m+1} R m\right|^{2} \cdot\left|\nabla^{m} R m\right|^{p-2}+C \int\left|\nabla^{m} R m\right|^{p}+C\left|\nabla^{m} R m\right|_{\left(g(t), L^{p}(\Omega)\right)}^{p-1} .
$$


To estimate $I_{3}$,

$$
\begin{aligned}
I_{3} \leq & C \int\left|\nabla^{m} R m\right|^{p}\left|\chi^{2} R m\right| \\
+ & C \sum_{i+j+k=m, j, k \leq m-1} \int\left|\nabla^{m} R m\right|^{p-1}\left|\nabla^{i}\left(\chi^{2}\right)\right|\left|\nabla^{j} R m\right|\left|\nabla^{k} R m\right| \\
\leq & C \int\left|\nabla^{m} R m\right|^{p}+C \sum_{i_{1}+i_{2}+j+k=m, j, k \leq m-1} \\
& \cdot\left[\left(\int\left|\nabla^{m} R m\right|^{p-1}\right)^{\frac{p-1}{p}} \cdot\left(\int\left|\nabla^{i_{1}} \chi\right|^{4 p}\right)^{\frac{1}{4 p}}\right. \\
& \left.\cdot\left(\int\left|\nabla^{i_{2}} \chi\right|^{4 p}\right)^{\frac{1}{4 p}} \cdot\left(\int\left|\nabla^{j} R m\right|^{4 p}\right)^{\frac{1}{4 p}}\left(\int\left|\nabla^{k} R m\right|^{4 p}\right)^{\frac{1}{4 p}}\right] .
\end{aligned}
$$

By induction assumption and the assumption of the proposition,

$$
I_{3} \leq C \int\left|\nabla^{m} R m\right|^{p}+C\left|\nabla^{m} R m\right|_{\left(g(t), L^{p}(\Omega)\right)}^{p-1} .
$$

For $I_{1}$, integration by parts yields

$$
I_{1} \leq 2 \int\left|\nabla^{m} R m\right|^{p-1} \chi|\nabla \chi|\left|\nabla^{m+1} R m\right|-\int \chi^{2}\left|\nabla^{m} R m\right|^{p-2}\left|\nabla^{m+1} R m\right|^{2} .
$$

Using the Hölder and Schwartz inequalities,

$$
I_{1} \leq-\frac{15}{16} \int \chi^{2}\left|\nabla^{m} R m\right|^{p-2}\left|\nabla^{m+1} R m\right|^{2}+C \int\left|\nabla^{m} R m\right|^{p} .
$$

By (4.8), (4.9), (4.10) and (4.1),

$$
\begin{aligned}
\frac{\partial}{\partial t} \int\left|\nabla^{m} R m\right|^{p} \leq & -\frac{3}{4} \int \chi^{2}\left|\nabla^{m} R m\right|^{p-2}\left|\nabla^{m+1} R m\right|^{2} \\
& +C \int\left|\nabla^{m} R m\right|^{p}+C\left|\nabla^{m} R m\right|_{\left(g(t), L^{p}(\Omega)\right)}^{p-1} .
\end{aligned}
$$

By Young's inequality,

$$
\frac{\partial}{\partial t} \int\left|\nabla^{m} R m\right|^{p} \leq C\left(\int\left|\nabla^{m} R m\right|^{p}+1\right) .
$$

Then $\int\left|\nabla^{m} R m\right|_{g(t)}^{p} \leq\left[\int\left|\nabla^{m} R m\right|_{g_{0}}^{p}+1\right] e^{C T}-1, \quad t \in[0, T)$. Hence $\left|\nabla^{m} R m\right|_{\left(g(t), L^{p}(\Omega)\right)}$ is uniformly bounded on $[0, T)$.

Theorem 4.6. If $g(t), t \in[0, T), 0<T<+\infty$ is a smooth solution of the local Ricci flow on $\left(M^{n}, g_{0}\right)$, assume $\left|\chi^{2} R m\right|_{(g(t), \infty)}$ is uniformly bounded on $[0, T)$. Then for any $m \geq 0,\left|\nabla^{m}\left(\chi^{2} R m\right)\right|_{(g(t), \infty)}$ is uniformly bounded on $[0, T)$.

Proof. By Lemma 4.3, if $|\nabla \chi|_{(g(t), \infty)}$ and $C_{s}(\Omega, t)$ are uniformly bounded on $[0, T)$, and $g(t)$ are uniformly equivalent to each other, then it suffices to prove

$(C 1)$ "For any $p>100, m \geq 0,\left|\nabla^{m}\left(\chi^{2} R m\right)\right|_{\left(g(t), L^{p}(\Omega)\right)}$ is uniformly bounded on $[0, T) . "$

We prove $(C 1)$ by induction on $m$. When $m=0$, the conclusion follows from the assumption of the theorem. Assume

$(A 1)$ "For any $p>100,\left|\nabla^{l}\left(\chi^{2} R m\right)\right|_{\left(g(t), L^{p}(\Omega)\right)}$ is uniformly bounded on $[0, T)$, where $0 \leq l \leq m-1$ and $m \geq 1$." 
We will prove

$(C 2)$ "For any $p>100,\left|\nabla^{m}\left(\chi^{2} R m\right)\right|_{\left(g(t), L^{p}(\Omega)\right)}$ is uniformly bounded on $[0, T) . "$ First we claim:

(C3) "For any $p>100, \int\left|\nabla^{k} \chi\right|^{p}$ is uniformly bounded on $[0, T)$, where $0 \leq k \leq$ $m . "$

We prove $(C 3)$ by induction on $k$. When $k=0$, the conclusion follows from the fact that $g(t)$ are uniformly equivalent to each other under the assumption in the theorem. Assume

$(A 2)$ "For any $q>100, \int\left|\nabla^{i} \chi\right|^{q}$ is uniformly bounded on $[0, T)$, where $0 \leq i \leq$ $k-1 . "$

Note $k \leq m$ in our claim. Using Lemma 3.6,

$$
\begin{aligned}
\frac{\partial}{\partial t} \int\left|\nabla^{k} \chi\right|^{p} \leq & C \sum_{1 \leq i \leq k-1}\left(\int\left|\nabla^{k} \chi\right|^{p}\right)^{\frac{p-1}{p}} \cdot\left(\int\left|\nabla^{i}\left(\chi^{2} R m\right)\right|^{2 p}\right)^{\frac{1}{2 p}} \cdot\left(\int\left|\nabla^{k-i} \chi\right|^{2 p}\right)^{\frac{1}{2 p}} \\
& +C \int\left|\nabla^{k} \chi\right|^{p} \cdot\left|\chi^{2} R m\right| \\
\leq & C\left|\nabla^{k} \chi\right|_{\left(g(t), L^{p}(\Omega)\right)}^{p-1}+C \int\left|\nabla^{k} \chi\right|^{p} \leq C\left(\int\left|\nabla^{k} \chi\right|^{p}+1\right) .
\end{aligned}
$$

For the last two inequalities, we used the assumption of the theorem, $(A 1),(A 2)$ and Young's inequality. Hence $\int\left|\nabla^{k} \chi\right|_{g(t)}^{p} d V_{g(t)}$ is uniformly bounded on $[0, T)$. From the induction method, claim $(C 3)$ is proved.

From the assumption of the theorem, $(C 3)$ and Proposition 4.5 , we get

$(C 4)$ "For any $p>100,\left|\nabla^{i} R m\right|_{g(t), L^{p}(\Omega)}$ is uniformly bounded on $[0, T)$, where $0 \leq i \leq m-1$."

By Lemma 3.6 and integration by parts,

$$
\frac{\partial}{\partial t} \int\left|\nabla^{m}\left(\chi^{2} R m\right)\right|^{p} \leq I_{1}+I_{2}+I_{3}+I_{4}
$$

where

$$
\begin{gathered}
I_{1}=-\int \chi^{2}\left|\nabla^{m+1}\left(\chi^{2} R m\right)\right|^{2}\left|\nabla^{m}\left(\chi^{2} R m\right)\right|^{p-2}, \\
I_{2}=\sum_{i+j+k=m+2,} \int\left|\nabla^{m}\left(\chi^{2} R m\right)\right|^{p-2}\left\langle\nabla^{m}\left(\chi^{2} R m\right), \nabla^{i} \chi * \nabla^{j} \chi * \nabla^{k}\left(\chi^{2} R m\right)\right\rangle, \\
I_{3}=\sum_{i+j+k=m, k \leq m-1} \int\left|\nabla^{m}\left(\chi^{2} R m\right)\right|^{p-2}\left\langle\nabla^{m}\left(\chi^{2} R m\right), \nabla^{i}\left(\chi^{2}\right) * \nabla^{j}\left(\chi^{2} R m\right) * \nabla^{k}(R m)\right\rangle, \\
I_{4}=\int\left|\nabla^{m}\left(\chi^{2} R m\right)\right|^{p-2}\left\langle\nabla^{m}\left(\chi^{2} R m\right), \chi^{2} R m * \nabla^{m}\left(\chi^{2} R m\right)\right\rangle .
\end{gathered}
$$

To estimate $I_{2}$,

$$
\begin{aligned}
& I_{2}=\int\left|\nabla^{m}\left(\chi^{2} R m\right)\right|^{p-2}\left\langle\nabla^{m}\left(\chi^{2} R m\right), \chi \nabla^{m+2} \chi *\left(\chi^{2} R m\right)\right\rangle \\
& +\int\left|\nabla^{m}\left(\chi^{2} R m\right)\right|^{p-2}\left\langle\nabla^{m}\left(\chi^{2} R m\right), \nabla \chi * \nabla^{m+1} \chi *\left(\chi^{2} R m\right)\right\rangle \\
& +\int\left|\nabla^{m}\left(\chi^{2} R m\right)\right|^{p-2}\left\langle\nabla^{m}\left(\chi^{2} R m\right), \chi \nabla^{m+1} \chi * \nabla\left(\chi^{2} R m\right)\right\rangle \\
& \quad+\int\left|\nabla^{m}\left(\chi^{2} R m\right)\right|^{p-2}\left\langle\nabla^{m}\left(\chi^{2} R m\right), \chi \nabla \chi * \nabla^{m+1}\left(\chi^{2} R m\right)\right\rangle
\end{aligned}
$$




$$
\begin{gathered}
+\sum_{i+j+k=m+2, i, j \leq m, k \leq m-1} \int\left|\nabla^{m}\left(\chi^{2} R m\right)\right|^{p-2} \\
\cdot\left\langle\nabla^{m}\left(\chi^{2} R m\right), \nabla^{i} \chi * \nabla^{j} \chi * \nabla^{k}\left(\chi^{2} R m\right)\right\rangle \\
+\int\left|\nabla^{m}\left(\chi^{2} R m\right)\right|^{p-2}\left\langle\nabla^{m}\left(\chi^{2} R m\right),\left(\chi \nabla^{2} \chi+\nabla \chi * \nabla \chi\right) * \nabla^{m}\left(\chi^{2} R m\right)\right\rangle .
\end{gathered}
$$

Using integration by parts,

$$
I_{2} \leq \Gamma_{1}+\Gamma_{2}+\Gamma_{3}+\Gamma_{4}+\Gamma_{5},
$$

where

$$
\begin{gathered}
\Gamma_{1}=C \int\left|\nabla^{m}\left(\chi^{2} R m\right)\right|^{p-2} \cdot\left|\nabla^{m+1}\left(\chi^{2} R m\right)\right| \cdot \chi\left|\nabla^{m+1} \chi\right| \cdot\left|\chi^{2} R m\right|, \\
\Gamma_{2}=C \int\left|\nabla^{m}\left(\chi^{2} R m\right)\right|^{p-1} \cdot|\nabla \chi| \cdot\left|\nabla^{m+1} \chi\right| \cdot\left|\chi^{2} R m\right|, \\
\Gamma_{3}=\left.\left|\int\right| \nabla^{m}\left(\chi^{2} R m\right)\right|^{p-2}\left\langle\nabla^{m}\left(\chi^{2} R m\right), \chi \nabla^{m+1} \chi * \nabla\left(\chi^{2} R m\right)\right\rangle \mid, \\
\Gamma_{4}=C \int \chi\left|\nabla^{m+1}\left(\chi^{2} R m\right)\right| \cdot\left|\nabla^{m}\left(\chi^{2} R m\right)\right|^{p-1}+C \int\left|\nabla^{m}\left(\chi^{2} R m\right)\right|^{p}, \\
\sum_{5}=C \sum_{i+j+k=m+2, i, j \leq m, k \leq m-1} \int\left|\nabla^{m}\left(\chi^{2} R m\right)\right|^{p-2} \cdot\left|\nabla^{i} \chi\right| \cdot\left|\nabla^{j} \chi\right| \cdot\left|\nabla^{k}\left(\chi^{2} R m\right)\right| .
\end{gathered}
$$

Using the Hölder and Young inequalities,

$$
\begin{aligned}
\Gamma_{1} \leq & \frac{1}{16} \int \chi^{2}\left|\nabla^{m}\left(\chi^{2} R m\right)\right|^{p}\left|\nabla^{m+1}\left(\chi^{2} R m\right)\right|^{2}+C \int\left|\nabla^{m}\left(\chi^{2} R m\right)\right|^{p} \\
& +C \int\left|\nabla^{m+1} \chi\right|^{p} .
\end{aligned}
$$

We can also estimate $\Gamma_{2}, \Gamma_{4}$ and $\Gamma_{5}$ in a similar way:

$$
\begin{gathered}
\Gamma_{2} \leq C \int\left|\nabla^{m}\left(\chi^{2} R m\right)\right|^{p}+C \int\left|\nabla^{m+1} \chi\right|^{p}, \\
\Gamma_{4} \leq \frac{1}{16} \int \chi^{2}\left|\nabla^{m}\left(\chi^{2} R m\right)\right|^{p}\left|\nabla^{m+1}\left(\chi^{2} R m\right)\right|^{2}+C \int\left|\nabla^{m}\left(\chi^{2} R m\right)\right|^{p}, \\
\Gamma_{5} \leq C \int\left|\nabla^{m}\left(\chi^{2} R m\right)\right|^{p}+C \sum_{i+j+k=m+2, i, j \leq m, k \leq m-1}\left[\int\left|\nabla^{i} \chi\right|^{3 p}\right. \\
\left.+\int\left|\nabla^{j} \chi\right|^{3 p}+\int\left|\nabla^{k}\left(\chi^{2} R m\right)\right|^{3 p}\right] \\
\leq C \int\left|\nabla^{m}\left(\chi^{2} R m\right)\right|^{p}+C .
\end{gathered}
$$

The last inequality in estimating $\Gamma_{5}$ follows from $(A 1)$ and $(C 3)$.

To estimate $\Gamma_{3}$, there are two cases: Case (1) and Case (2).

Case (1): If $m=1$, using integration by parts,

$$
\Gamma_{3} \leq C \int\left|\nabla^{m}\left(\chi^{2} R m\right)\right|^{p-1}\left|\nabla^{m+1}\left(\chi^{2} R m\right)\right|(\chi|\nabla \chi|)+C \int\left|\nabla^{m}\left(\chi^{2} R m\right)\right|^{p}|\nabla \chi|^{2} .
$$

Using the fact that $|\nabla \chi|_{(g(t), \infty)}$ is uniformly bounded, we get that if $m=1$,

$$
\Gamma_{3} \leq \frac{1}{16} \int \chi^{2}\left|\nabla^{m}\left(\chi^{2} R m\right)\right|^{p}\left|\nabla^{m+1}\left(\chi^{2} R m\right)\right|^{2}+C \int\left|\nabla^{m}\left(\chi^{2} R m\right)\right|^{p} .
$$

Case (2): If $m \geq 2$, using integration by parts,

$$
\begin{aligned}
\Gamma_{3} \leq & C \int\left|\nabla^{m}\left(\chi^{2} R m\right)\right|^{p-2} \cdot\left|\nabla^{m+1}\left(\chi^{2} R m\right)\right| \cdot\left|\nabla\left(\chi^{2} R m\right)\right| \cdot \chi\left|\nabla^{m} \chi\right| \\
& +C \int\left|\nabla^{m}\left(\chi^{2} R m\right)\right|^{p-1}|\nabla \chi|\left|\nabla^{m} \chi\right|\left|\nabla\left(\chi^{2} R m\right)\right|+\Gamma_{3,1},
\end{aligned}
$$


where

$$
\Gamma_{3,1}=\left.\left|\int\right| \nabla^{m}\left(\chi^{2} R m\right)\right|^{p-2}\left\langle\nabla^{m}\left(\chi^{2} R m\right), \chi \nabla^{m} \chi * \nabla^{2}\left(\chi^{2} R m\right)\right\rangle \mid .
$$

Using the Hölder and Young inequalities,

$$
\begin{aligned}
\Gamma_{3} \leq & \frac{1}{16} \int \chi^{2}\left|\nabla^{m}\left(\chi^{2} R m\right)\right|^{p}\left|\nabla^{m+1}\left(\chi^{2} R m\right)\right|^{2}+C \int\left|\nabla^{m}\left(\chi^{2} R m\right)\right|^{p} \\
& +C \int\left|\nabla\left(\chi^{2} R m\right)\right|^{2 p}+C \int\left|\nabla^{m} \chi\right|^{2 p}+\Gamma_{3,1} .
\end{aligned}
$$

Note that $m \geq 2$; using $(A 1)$ and $(C 3)$,

$$
\Gamma_{3} \leq \frac{1}{16} \int \chi^{2}\left|\nabla^{m}\left(\chi^{2} R m\right)\right|^{p}\left|\nabla^{m+1}\left(\chi^{2} R m\right)\right|^{2}+C \int\left|\nabla^{m}\left(\chi^{2} R m\right)\right|^{p}+C+\Gamma_{3,1} .
$$

To estimate $\Gamma_{3,1}$, note that $m \geq 2$. There are still two cases: Case (2.1) and Case (2.2).

Case (2.1): If $m=2$, using integration by parts and the fact that $|\nabla \chi|_{(g(t), \infty)}$ is uniformly bounded,

$$
\Gamma_{3,1} \leq \frac{1}{16} \int \chi^{2}\left|\nabla^{m}\left(\chi^{2} R m\right)\right|^{p}\left|\nabla^{m+1}\left(\chi^{2} R m\right)\right|^{2}+C \int\left|\nabla^{m}\left(\chi^{2} R m\right)\right|^{p} .
$$

Case (2.2): If $m \geq 3$, using the Hölder and Young inequalities,

$$
\begin{aligned}
\Gamma_{3,1} & \leq C \int\left|\nabla^{m}\left(\chi^{2} R m\right)\right|^{p}+C \int\left|\nabla^{m} \chi\right|^{2 p}+C \int\left|\nabla^{2}\left(\chi^{2} R m\right)\right|^{2 p} \\
& \leq C \int\left|\nabla^{m}\left(\chi^{2} R m\right)\right|^{p}+C .
\end{aligned}
$$

The last inequality follows from $(A 1)$ and $(C 3)$.

By (4.19) and (4.20),

$$
\Gamma_{3,1} \leq \frac{1}{16} \int \chi^{2}\left|\nabla^{m}\left(\chi^{2} R m\right)\right|^{p}\left|\nabla^{m+1}\left(\chi^{2} R m\right)\right|^{2}+C \int\left|\nabla^{m}\left(\chi^{2} R m\right)\right|^{p}+C .
$$

From (4.18) and (4.21), we get that if $m \geq 2$,

$$
\Gamma_{3} \leq \frac{1}{8} \int \chi^{2}\left|\nabla^{m}\left(\chi^{2} R m\right)\right|^{p}\left|\nabla^{m+1}\left(\chi^{2} R m\right)\right|^{2}+C \int\left|\nabla^{m}\left(\chi^{2} R m\right)\right|^{p}+C .
$$

From (4.17) and (4.22), when $m \geq 1$, (4.22) holds.

By (4.12), (4.13), (4.14), (4.15), (4.16) and (4.22),

$$
\begin{aligned}
I_{2} \leq & \frac{1}{4} \int \chi^{2}\left|\nabla^{m}\left(\chi^{2} R m\right)\right|^{p}\left|\nabla^{m+1}\left(\chi^{2} R m\right)\right|^{2}+C \int\left|\nabla^{m}\left(\chi^{2} R m\right)\right|^{p} \\
& +C \int\left|\nabla^{m+1} \chi\right|^{p}+C .
\end{aligned}
$$

To estimate $I_{3}$,

$$
\begin{aligned}
I_{3} \leq & C \sum_{i_{1}+i_{2}+j+k=m, k \leq m-1, j \leq m-1}\left(\int\left|\nabla^{m}\left(\chi^{2} R m\right)\right|^{p}\right)^{\frac{1}{p}} \cdot\left(\int\left|\nabla^{i_{1}} \chi\right|^{4 p}\right)^{\frac{1}{4 p}} \\
& \left.\cdot\left(\int\left|\nabla^{i_{2}} \chi\right|^{4 p}\right)^{\frac{1}{4 p}} \cdot\left(\int\left|\nabla^{j}\left(\chi^{2} R m\right)\right|^{4 p}\right)^{\frac{1}{4 p}} \cdot\left(\int\left|\nabla^{k} R m\right|^{4 p}\right)^{\frac{1}{4 p}}\right] \\
& +C \int\left|\nabla^{m}\left(\chi^{2} R m\right)\right|^{p} .
\end{aligned}
$$

Hence from $(A 1),(C 3),(C 4)$ and Young's inequality,

$$
I_{3} \leq C \int\left|\nabla^{m}\left(\chi^{2} R m\right)\right|^{p}+C
$$

From the assumption of the theorem,

$$
I_{4} \leq C \int\left|\nabla^{m}\left(\chi^{2} R m\right)\right|^{p} .
$$


By (4.23), (4.24), (4.25) and (4.11),

$$
\begin{aligned}
\frac{\partial}{\partial t} \int\left|\nabla^{m}\left(\chi^{2} R m\right)\right|^{p} \leq & -\frac{3}{4} \int \chi^{2}\left|\nabla^{m}\left(\chi^{2} R m\right)\right|^{p}\left|\nabla^{m+1}\left(\chi^{2} R m\right)\right|^{2} \\
& +C \int\left|\nabla^{m}\left(\chi^{2} R m\right)\right|^{p}+C \int\left|\nabla^{m+1} \chi\right|^{p}+C .
\end{aligned}
$$

By Lemma 3.6

$$
\begin{aligned}
& \frac{\partial}{\partial t} \int\left|\nabla^{m+1} \chi\right|^{p} \\
& \leq C \int\left|\nabla^{m+1} \chi\right|^{p} \cdot\left|\chi^{2} R c\right|+C \int\left|\nabla^{m+1} \chi\right|^{p-1} \cdot\left|\nabla^{m}\left(\chi^{2} R m\right)\right| \cdot|\nabla \chi| \\
&+C \sum_{1 \leq k \leq m-1} \int\left|\nabla^{m+1} \chi\right|^{p-1} \cdot\left|\nabla^{k}\left(\chi^{2} R m\right)\right| \cdot\left|\nabla^{m+1-k} \chi\right| \\
& \leq C \int\left|\nabla^{m+1} \chi\right|^{p}+C\left(\int\left|\nabla^{m+1} \chi\right|^{p}\right)^{\frac{1}{p}} \cdot\left(\int\left|\nabla^{m}\left(\chi^{2} R m\right)\right|^{p}\right)^{\frac{1}{p}} \\
&+C \sum_{1 \leq k \leq m-1}\left[\left(\int\left|\nabla^{m+1} \chi\right|^{p}\right)^{\frac{1}{p}} \cdot\left(\int\left|\nabla^{k} \chi\right|^{2 p}\right)^{\frac{1}{2 p}} \cdot\left(\int\left|\nabla^{m+1-k} \chi\right|^{2 p}\right)^{\frac{1}{2 p}}\right] .
\end{aligned}
$$

The last inequality follows from the Hölder inequality and the assumption of the theorem.

Hence by Young's inequality, $(A 1)$ and $(C 3)$,

$$
\frac{\partial}{\partial t} \int\left|\nabla^{m+1} \chi\right|^{p} \leq C_{1} \int\left|\nabla^{m+1} \chi\right|^{p}+C_{2} \int\left|\nabla^{m}\left(\chi^{2} R m\right)\right|^{p}+C_{3} .
$$

By (4.27) and Lemma 4.1] on $[0, T)$,

$$
\begin{aligned}
\int\left|\nabla^{m+1} \chi\right|_{g(t)}^{p} d V_{g(t)} \leq & e^{C_{1} t}\left[\int\left|\nabla^{m+1} \chi\right|_{g_{0}}^{p} d V_{g_{0}}\right. \\
& \left.+\int_{0}^{t} e^{-C_{1} s}\left(C_{3}+C_{2}\left|\nabla^{m}\left(\chi^{2} R m\right)\right|_{\left(g(s), L^{p}(\Omega)\right)}^{p}\right) d s\right] .
\end{aligned}
$$

Hence on $[0, T)$,

$$
\int\left|\nabla^{m+1} \chi\right|^{p} \leq C+C \int_{0}^{t}\left|\nabla^{m}\left(\chi^{2} R m\right)\right|_{\left(g(s), L^{p}(\Omega)\right)}^{p} d s .
$$

By (4.26) and (4.28),

$$
\begin{aligned}
\frac{\partial}{\partial t}\left|\nabla^{m}\left(\chi^{2} R m\right)\right|_{\left(g(t), L^{p}(\Omega)\right)}^{p} \leq & C_{4} \int_{0}^{t}\left|\nabla^{m}\left(\chi^{2} R m\right)\right|_{\left(g(s), L^{p}(\Omega)\right)}^{p} d s \\
& +C_{5}\left|\nabla^{m}\left(\chi^{2} R m\right)\right|_{\left(g(t), L^{p}(\Omega)\right)}^{p}+C_{6} .
\end{aligned}
$$

By (4.29) and Lemma 4.2, $\left|\nabla^{m}\left(\chi^{2} R m\right)\right|_{\left(g(t), L^{p}(\Omega)\right)}^{p}$ is uniformly bounded on $[0, T)$. Hence $(C 2)$ is proved. By the induction method, $(C 1)$ is proved.

Proposition 4.7. If $g(t), t \in[0, T)$ is a solution to the local Ricci flow on $\left(M^{n}, g_{0}\right)$, and for any $m \geq 0,\left|\nabla^{m}\left(\chi^{2} R m\right)\right|_{(g(t), \infty)}$ is uniformly bounded on $[0, T)$. Then the local Ricci flow can be extended smoothly through time $T$.

Proof. Using a similar argument to one in section 7 of chapter 6 of [1, the difference is that $\nabla^{m}\left(\chi^{2} R m\right)$ plays the key role in our local Ricci flow case instead of $\nabla^{m} R m$ in Ricci flow case. 
Theorem 4.8. If $g(t), t \in[0, T), 0<T<+\infty$ is a smooth solution of the local Ricci flow on $\left(M^{n}, g_{0}\right)$. Assume $\left|\chi^{2} R m\right|_{(g(t), \infty)}$ is uniformly bounded on $[0, T)$, then the local Ricci flow can be extended smoothly through time T.

Proof. By Theorem 4.6 and Proposition 4.7, we get our conclusion.

\section{Existence time estimates (The first Case: $p_{0}>\frac{n}{2}$ )}

In this section, we use the product of two different cut-off functions to get curvature estimates, so we call them "local local" curvature estimates. Also in this section, we assume that $\left(M^{n}, g_{0}\right)$ is an $n$-dimensional $(n \geq 3)$ complete noncompact Riemannian manifold satisfying the following assumptions:

$$
\left\{\begin{aligned}
\left(f_{B_{x}\left(4 r_{0}\right)} h^{\frac{2 n}{n-2}} d V_{g_{0}}\right)^{\frac{n-2}{n}} & \leq A_{0} r_{0}^{2} f_{B_{x}\left(4 r_{0}\right)}|\nabla h|^{2} d V_{g_{0}}, \\
\left(f_{B_{x}\left(r_{0}\right)}\left|R m\left(g_{0}\right)\right|^{p_{0}} d V_{g_{0}}\right)^{\frac{1}{p_{0}}} & \leq K_{1}
\end{aligned}\right.
$$

for any $x \in M$ and $h \in C_{0}^{\infty}\left(B_{x}\left(4 r_{0}\right)\right)$, where $A_{0} \geq 1, p_{0}>\frac{n}{2}, K_{1}, r_{0}$ are some positive constants, and $B_{x}(r)$ is the open geodesic ball of radius $r$ centered at $x$ on $\left(M, g_{0}\right)$. Also, $f$ means the usual average of the integrand.

We always assume $\|\nabla \chi\|_{\infty} \triangleq\|\nabla \chi\|_{\left(g_{0}, \infty\right)}=\sup _{x \in \Omega}\left(g_{0}^{i j} \nabla_{i} \chi \nabla_{j} \chi\right)^{\frac{1}{2}} \leq 1$. Now we state some lemmas about local Sobolev constants under the scaling of metrics. There is the well-known local Sobolev inequality

$$
\left(f_{B_{x}(r)} f^{\frac{2 n}{n-2}} d V_{g(t)}\right)^{\frac{n-2}{n}} \leq A\left(B_{x}(r), t\right) r^{2} f_{B_{x}(r)}|\nabla f|^{2} d V_{g(t)}, \quad \forall f \in C_{0}^{\infty}\left(B_{x}(r)\right)
$$

with respect to each metric $g(t)$, and $A\left(B_{x}(r), t\right)$ depends on both $t$ and $B_{x}(r)$. Note that in (5.2), although $B_{x}(r)$ is the geodesic ball with respect to $g_{0}, f_{B_{x}(r)} f^{\frac{2 n}{n-2}} d V_{g(t)}$ $\triangleq \frac{1}{V_{g(t)}\left(B_{x}(r)\right)} \int_{B_{x}(r)} f^{\frac{2 n}{n-2}} d V_{g(t)}$. When $r$ is fixed, $A(t) \triangleq \sup _{x \in M^{n}} A\left(B_{x}(r), t\right)$.

Lemma 5.1. If $a^{-1} g_{0} \leq g(t) \leq a g_{0}$, then

$$
a^{-(n+1)} A_{0} \leq A(t) \leq a^{n+1} A_{0},
$$

where $A_{0}=A(0)$ is with respect to $g_{0}$, and $a \geq 1$ is some positive constant.

Proof. It is straightforward from the scaling argument and (5.2).

Remark 5.2. We have defined two Sobolev constants. The Sobolev constant for $(\Omega, g(t))$ is $C_{s}(\Omega, g(t))$ defined in (2.6), and local Sobolev constant $A\left(B_{x}(r), t\right)$ is defined in (5.2). They are different from each other, although they satisfy a similar property as in Lemma 5.1

Lemma 5.3. Assume the inequality

$$
\left(f_{B_{x}\left(4 r_{0}\right)} h^{\frac{2 n}{n-2}} d V_{g_{0}}\right)^{\frac{n-2}{n}} \leq A_{0} r_{0}^{2} f_{B_{x}\left(4 r_{0}\right)}|\nabla h|^{2} d V_{g_{0}},
$$

where $h \in C_{0}^{\infty}\left(B_{x}\left(4 r_{0}\right)\right), A_{0} \geq 1$ and $r_{0}$ are fixed positive constants. Then

$$
V_{g_{0}}\left(B_{x}(\rho)\right) \geq N_{1}\left(\frac{\rho}{4 r_{0}}\right)^{n} V_{g_{0}}\left(B_{x}\left(4 r_{0}\right)\right), \quad 0<\rho \leq 4 r_{0},
$$

where $N_{1}=2^{2 n-\frac{n^{2}}{2}} A_{0}^{-\frac{n}{2}}$ and $N_{1} \leq 1$. 
Proof. For simplicity, we use $V\left(4 r_{0}\right)$ instead of $V_{g_{0}}\left(B_{x}\left(4 r_{0}\right)\right)$ in the proof. By assumption,

$$
\left(\int_{B\left(4 r_{0}\right)}|h|^{\frac{2 n}{n-1}}\right)^{\frac{n-2}{2 n}} \leq A_{0}^{\frac{1}{2}} r_{0} V\left(4 r_{0}\right)^{-\frac{1}{n}}\left(\int_{B\left(4 r_{0}\right)}|\nabla h|^{2}\right)^{\frac{1}{2}} .
$$

We recall Theorem 3.1.5 in [11].

Theorem. Assume that the inequality

$$
\forall f \in C_{0}^{\infty}(M), \quad\|f\|_{r} \leq\left(C\|\nabla f\|_{p}\right)^{\theta}\|f\|_{s}^{1-\theta}
$$

is satisfied for some $r, s, \theta$ with $0<s \leq r \leq \infty$ and $0<\theta \leq 1$. Assume also that

$$
\frac{\theta}{p}+\frac{1-\theta}{s}-\frac{1}{r}>0 \text {. }
$$

Then $V(x, \rho) \geq c \rho^{\nu}$ with $\nu$ defined by

$$
\frac{\theta}{\nu}=\frac{\theta}{p}+\frac{1-\theta}{s}-\frac{1}{r}
$$

and the constant $c$ given by $c=2^{-\frac{\nu^{2}}{\theta r}-\frac{\nu}{\theta}} C^{-\nu}$.

Let $\theta=1, s=r=\frac{2 n}{n-2}, p=2$ in the above theorem. Also, $C=\frac{A_{0}^{\frac{1}{2}} r_{0}}{V^{\frac{1}{n}}}$, where $V=V\left(4 r_{0}\right)$, and $\nu=n$. Then $c=2^{-\frac{n^{2}}{2}}\left(A_{0}^{\frac{1}{2}} r_{0}\right)^{-n} V$, and by the above theorem,

$$
V(\rho) \geq c \rho^{\nu}=2^{2 n-\frac{n^{2}}{2}} A_{0}^{-\frac{n}{2}}\left(\frac{\rho}{4 r_{0}}\right)^{n} V \geq N_{1}\left(\frac{\rho}{4 r_{0}}\right)^{n} V\left(4 r_{0}\right) .
$$

Lemma 5.4. $\left(M^{n}, g\right)$ is a smooth, complete Riemannian manifold with the property:

$$
V_{g}\left(B_{x}(\rho)\right) \geq N_{1}\left(\frac{\rho}{4 r_{0}}\right)^{n} \operatorname{Vol}\left(B_{x}\left(4 r_{0}\right)\right), \quad 0<\rho \leq 4 r_{0}, \quad \forall x \in M,
$$

where $r_{0}$ and $N_{1}$ are fixed positive constants. Then there exists a sequence $\left(x_{i}\right)$ of points of $M$ such that

(I) $M=\bigcup_{i=1}^{\infty} B_{x_{i}}\left(r_{0}\right)$.

(II) For any $i \neq j, B_{x_{i}}\left(\frac{r_{0}}{2}\right) \cap B_{x_{j}}\left(\frac{r_{0}}{2}\right)=\emptyset$.

(III) For any $j, B_{x_{j}}\left(r_{0}\right)$ intersects at most $N$ balls $B_{x_{i}}\left(r_{0}\right), i \neq j$, where $N=$ $2^{4 n} N_{1}^{-2}=2^{n^{2}} A_{0}^{n}$.

Proof. Let $X_{r_{0}}=\left\{\left(x_{i}\right)_{I}, x_{i} \in M\right.$, s.t. $I$ is countable and $\left.\forall i \neq j, d_{g}\left(x_{i}, x_{j}\right) \geq r_{0}\right\}$. As one can easily check, $X_{r_{0}}$ is partially ordered by inclusion and every chain in $X_{r_{0}}$ has an upper bound. Hence, by Zorn's lemma, $X_{r_{0}}$ contains a maximal element $\left(x_{i}\right)$ and $\left(x_{i}\right)$ satisfies (I) and (II). From now on, let $\left(x_{i}\right)$ be such that (I) and (II) are satisfied. For $x \in M$, we define

$$
I_{2 r_{0}}(x)=\left\{i: x \in B_{x_{i}}\left(2 r_{0}\right)\right\} .
$$

Then

$$
V\left(B_{x}\left(2 r_{0}\right)\right) \geq \frac{N_{1}}{2^{n}} V\left(B_{x}\left(4 r_{0}\right)\right) \geq \frac{N_{1}}{2^{n}} \sum_{i \in I_{2 r_{0}}(x)} V\left(B_{x_{i}}\left(\frac{r_{0}}{2}\right)\right)
$$


The last inequality follows from $\bigcup_{i \in I_{2 r_{0}}(x)} B_{x_{i}}\left(\frac{r_{0}}{2}\right) \subseteq B_{x}\left(4 r_{0}\right)$ and $B_{x_{i}}\left(\frac{r_{0}}{2}\right) \cap B_{x_{j}}\left(\frac{r_{0}}{2}\right)$ $=\emptyset$ if $i \neq j$. By the assumption, $V\left(B_{x_{i}}\left(\frac{r_{0}}{2}\right)\right) \geq \frac{N_{1}}{8^{n}} V\left(B_{x_{i}}\left(4 r_{0}\right)\right)$. Also, for $i \in$ $I_{2 r_{0}}(x), B_{x}\left(2 r_{0}\right) \subseteq B_{x_{i}}\left(4 r_{0}\right)$,

$$
V\left(B_{x_{i}}\left(\frac{r_{0}}{2}\right)\right) \geq \frac{N_{1}}{8^{n}} V\left(B_{x}\left(2 r_{0}\right)\right) .
$$

By (5.3) and (5.4),

$$
V\left(B_{x}\left(2 r_{0}\right)\right) \geq N_{1}^{2} 2^{-4 n} \operatorname{Card}\left(I_{2 r_{0}}(x)\right) V\left(B_{x}\left(2 r_{0}\right)\right),
$$

where $\operatorname{Card}$ stands for the cardinality. For any $x \in M, \operatorname{Card}\left(I_{2 r_{0}}(x)\right) \leq 2^{4 n} N_{1}^{-2}$. For fixed $x_{j}$, if $B_{x_{j}}\left(r_{0}\right) \cap B_{x_{i}}\left(r_{0}\right) \neq \emptyset$, then $x_{i} \in B_{x_{j}}\left(2 r_{0}\right), i \in I_{2 r_{0}}\left(x_{j}\right)$ and we get that $N \leq \operatorname{Card}\left(I_{2 r_{0}}\left(x_{j}\right)\right) \leq 2^{4 n} N_{1}^{-2}$

Lemma 5.5. For the covering $\left(B_{x_{i}}\left(r_{0}\right)\right)$ of $(M, g)$ as in Lemma 5.4, we can find $\xi_{i} \in C_{0}^{0,1}\left(B_{x_{i}}\left(r_{0}\right)\right), 0 \leq \xi_{i} \leq 1, \sum_{i} \xi_{i}^{2}=1,\left|\nabla \xi_{i}\right|^{2} \leq C\left(r_{0}, N\right)$, where $C\left(r_{0}, N\right)=$ $\frac{8}{r_{0}^{2}}(N+1)$ and $N$ is as in Lemma 5.4.

Proof. Let $\rho:[0, \infty) \rightarrow[0,1]$ be defined by

$$
\rho(t)=\left\{\begin{aligned}
1, & 0 \leq t \leq \frac{r_{0}}{2} \\
\left(2-\frac{2}{r_{0}} t\right)^{2}, & \frac{r_{0}}{2} \leq t \leq r_{0} \\
0, & r_{0} \leq t
\end{aligned}\right.
$$

Let $\alpha_{i}(x)=\rho\left(d_{g}\left(x, x_{i}\right)\right)$, where $d_{g}$ denotes the distance associated to $g$ and $x \in M$. Clearly, $\alpha_{i}$ is Lipschitz with compact support. Note that for any $m$, $\left|\nabla \alpha_{m}\right| \leq 2\left(2-\frac{2}{r_{0}} t\right) \frac{2}{r_{0}}=\frac{4}{r_{0}} \alpha_{m}^{\frac{1}{2}}$; that is, $\frac{\left|\nabla \alpha_{m}\right|^{2}}{\left|\alpha_{m}\right|} \leq\left(\frac{4}{r_{0}}\right)^{2}$. Let $\eta_{i}=\frac{\alpha_{i}}{\sum_{m} \alpha_{m}}$. Then $\eta_{i}$ is a partition of unity subordinate to the covering $\left(B_{x_{i}}\left(r_{0}\right)\right)$ :

$$
\begin{aligned}
\left|\nabla \eta_{i}\right| & \leq\left|\frac{\nabla \alpha_{i}}{\left(\sum_{m} \alpha_{m}\right)}\right|+\frac{\left|\alpha_{i}\right| \cdot\left|\sum_{m} \nabla \alpha_{m}\right|}{\left(\sum_{m} \alpha_{m}\right)^{2}} \leq\left|\nabla \alpha_{i}\right|+\frac{4 \eta_{i}}{r_{0}} \cdot \frac{\sum_{m} \alpha_{m}^{\frac{1}{2}}}{\sum_{m} \alpha_{m}} \\
& \leq\left|\nabla \alpha_{i}\right|+\frac{4 \eta_{i}}{r_{0}} \sqrt{N} .
\end{aligned}
$$

In the last equality, we use the Cauchy-Schwartz inequality and property (III) in Lemma 5.4 .

Let $\xi_{i}=\eta_{i}^{\frac{1}{2}}$; then $\xi_{i} \in C_{0}^{0,1}\left(B_{x_{i}}\left(r_{0}\right)\right), 0 \leq \xi_{i} \leq 1, \sum_{i} \xi_{i}^{2}=1$. Hence

$$
\left|\nabla \xi_{i}\right|^{2}=\frac{1}{4} \cdot \frac{\left|\nabla \eta_{i}\right|^{2}}{\left|\eta_{i}\right|} \leq \frac{1}{4\left|\alpha_{i}\right|} \cdot 2\left(\left|\nabla \alpha_{i}\right|^{2}+\left(\frac{4}{r_{0}} \eta_{i} \sqrt{N}\right)^{2}\right) \leq \frac{8}{r_{0}^{2}}(N+1) .
$$

For simplicity, we use the notation $B_{i}$ to replace $B_{x_{i}}\left(r_{0}\right)$ in the rest of this section. Note that $\left(x_{i}\right)$ are chosen as in Lemma 5.4 and are fixed in the rest of this section and in section 6 . We also use $\xi$ to replace $\xi_{i}$ in some proofs of this section; it will be clear from the context when we do such a replacement. We use the following notation in the rest of this section:

$$
f h d V_{g(t)} \triangleq \frac{1}{V_{g(t)}\left(B_{i}\right)} \int_{B_{i} \cap \Omega} h d V_{g(t)}
$$

for any $h \in C_{0}^{\infty}\left(B_{i}\right)$, and it will be clear from the context which $B_{i}$ we choose. 
From Theorem 2.9 and DeTurck's trick, local Ricci flow (1.2) has a smooth solution on a sufficiently small time interval starting at $t=0$. By (5.1), we can assume that $\left[0, T_{\max }\right)$ is the maximal time interval, on which local Ricci flow (1.2) has a smooth solution and such that the following hold for each metric $g(t)$ (where $\left.g(0)=g_{0}\right)$ :

$$
\begin{gathered}
\left(f_{B_{x}\left(4 r_{0}\right)} h^{\frac{2 n}{n-2}} d V_{g(t)}\right)^{\frac{n-2}{n}} \leq 4 A_{0} r_{0}^{2} f_{B_{x}\left(4 r_{0}\right)}|\nabla h|^{2} d V_{g(t)}, \\
\frac{1}{2} g_{0} \leq g(t) \leq 2 g_{0}, \\
\left(\frac{1}{V_{g(t)}\left(B_{i}\right)} \int_{B_{i} \cap \Omega}|R m(g(t))|^{p_{0}} d V_{g(t)}\right)^{\frac{1}{p_{0}}} \leq(2 N)^{\frac{1}{p_{0}}} K_{1}, \quad i=1,2,3, \cdots,
\end{gathered}
$$

for any $x \in M, h \in C_{0}^{\infty}\left(B_{x}\left(4 r_{0}\right)\right)$ and $B_{i}$ chosen as in Lemma 5.4 .

In the following lemma, we get the parabolic version of energy estimates on $\left[0, T_{\max }\right)$ under the local Ricci flow.

Lemma 5.6. For $0 \leq t<T_{\max }$, any $i, q \geq 1, p \geq \frac{n}{2}, p^{\prime} \geq 0$ and $p \geq p^{\prime}$, there exists some positive constant

$$
C_{1}\left(A_{0}, K_{1}, n, p_{0}, r_{0}\right)=C\left(n, p_{0}\right) A_{0}^{\frac{\left(2 p_{0}+3\right) n}{2 p_{0}-n}} K_{1}^{\frac{2 p_{0}}{2 p_{0}-n}}\left[r_{0}^{-2}+r_{0}^{\frac{2 n}{2 p_{0}-n}}\right]
$$

such that

$$
\begin{aligned}
& \frac{\partial}{\partial t}\left(f \xi_{i}^{2 q} \chi^{2 p^{\prime}}|R m|^{p} d V_{g(t)}\right)+\frac{1}{10}\left(f\left|\nabla\left(\xi_{i}^{q} \chi^{p^{\prime}+1}|R m|^{\frac{p}{2}}\right)\right|^{2} d V_{g(t)}\right) \\
& \leq C_{1}\left(A_{0}, K_{1}, n, p_{0}, r_{0}\right) p^{\frac{n}{2 p_{0}-n}+3} q^{2}\left(f \xi_{i}^{2 q-2} \chi^{2 p^{\prime}}|R m|^{p} d V_{g(t)}\right) .
\end{aligned}
$$

Proof. Set $f(x, t)=|R m(x)|_{g(t)}$. We get the following:

$$
\frac{\partial}{\partial t}\left(f \xi^{2 q} \chi^{2 p^{\prime}} f^{p}\right) \leq \sum_{k=1}^{7} I_{k}
$$

where

$$
\begin{gathered}
I_{1}=p f \xi^{2 q} \chi^{2 p^{\prime}+2} f^{p-1} \Delta f, \quad I_{2}=p f \xi^{2 q} \chi^{2 p^{\prime}+2} f^{p-2}|\nabla f|^{2}, \\
I_{3}=-(1-\epsilon) p f \xi^{2 q} \chi^{2 p^{\prime}+2} f^{p-2}|\nabla R m|^{2}, \\
I_{4}=10 p f \xi^{2 q} \chi^{2 p^{\prime}+2} f^{p+1}-f \xi^{2 q} \chi^{2 p^{\prime}+2} f^{p} R \\
I_{5}=\left(\frac{10^{5}}{\epsilon}\right) n p f \xi^{2 q} \chi^{2 p^{\prime}}|\nabla \chi|^{2} f^{p}, \\
I_{6}=8 p f \xi^{2 q} \chi^{2 p^{\prime}+1} f^{p-2} g^{r i} g^{s j} g^{p k} g^{q l} R_{r s p q} R_{j k} \chi_{i l}, \\
I_{7}=n(n-1)\left(f \xi^{2 q} \chi^{2 p^{\prime}} f^{p}\right) \cdot\left(\frac{1}{V_{g(t)}\left(B_{i}\right)} \int_{B_{i}} \chi^{2} f\right) .
\end{gathered}
$$

For simplification, we set the following notation:

$$
\begin{aligned}
& \tilde{a}=\xi^{q} \chi^{p^{\prime}} f^{\frac{p}{2}}|\nabla \chi|, \quad \tilde{b}=\xi^{q} \chi^{p^{\prime}+1} f^{\frac{p}{2}-1}|\nabla f|, \\
& \tilde{c}=\xi^{q} \chi^{p^{\prime}+1} f^{\frac{p}{2}-1}|\nabla R m|, \quad \tilde{d}=f\left|\nabla\left(\xi^{q} \chi^{p^{\prime}+1} f^{\frac{p}{2}}\right)\right|^{2}, \quad \tilde{e}=\xi^{q-1} \chi^{p^{\prime}+1} f^{\frac{p}{2}}|\nabla \xi| .
\end{aligned}
$$


Then

$$
\begin{aligned}
& I_{1} \leq-p(p-1) f \tilde{b}^{2}+2 \epsilon p f \tilde{b}^{2}+\frac{p q^{2}}{\epsilon} f \tilde{e}^{2}+\frac{p\left(p^{\prime}+1\right)^{2}}{\epsilon} f \tilde{a}^{2}, \\
& I_{2} \leq p f \tilde{b}^{2}, \quad I_{3}=-(1-\epsilon) p f \tilde{c}^{2}, \quad I_{5} \leq\left(\frac{10^{5}}{\epsilon}\right) n p f \tilde{a}^{2},
\end{aligned}
$$

where $0<\epsilon<\frac{1}{8}$ is some constant to be determined later.

Using integration by parts and Young's inequality,

$$
I_{6} \leq \epsilon p f \tilde{c}^{2}+\frac{1}{\epsilon} 10^{6} n^{3} p^{3} q^{2}\left(f \tilde{a}^{2}+f \tilde{e}^{2}\right) .
$$

Also,

$$
\begin{aligned}
I_{7} & \leq n(n-1) f \xi^{2 q} \chi^{2 p^{\prime}} f^{p} \cdot\left(\frac{1}{V_{g(t)}\left(B_{i}\right)} \int_{B_{i} \cap \Omega} f^{p_{0}}\right)^{\frac{1}{p_{0}}} \\
& \leq C\left(n, p_{0}\right) A_{0}^{\frac{n}{p_{0}}} K_{1}\left(f \xi_{i}^{2 q-2} \chi^{2 p^{\prime}} f^{p} d V_{g(t)}\right) .
\end{aligned}
$$

In the last inequality above, we use the fact that $N=2^{n^{2}} A_{0}^{n}$ by Lemma 5.3 and Lemma 5.4 .

Note that $|R| \leq n|R m| \leq 2 p f, p_{0}^{\prime}<\frac{n}{n-2}$, where $\frac{1}{p_{0}}+\frac{1}{p_{0}^{\prime}}=1$, and

$$
\begin{aligned}
I_{4} \leq & 12 p f \xi^{2 q} \chi^{2 p^{\prime}+2} f^{p+1} \leq 12 p \nVdash f \|_{p_{0}, B_{i} \cap \Omega} \cdot\left[f\left(\xi^{2 q} \chi^{2 p^{\prime}+2} f^{p}\right)^{p_{0}^{\prime}}\right]^{\frac{1}{p_{0}^{\prime}}} \\
\leq & 12 p(2 N)^{\frac{1}{p_{0}}} K_{1} \cdot\left[C\left(\epsilon, p_{0}, n\right)\left(2 N p K_{1} A_{0} r_{0}^{2}\right)^{\frac{n}{2 p_{0}-n}} f \xi^{2 q} \chi^{2 p^{\prime}+2} f^{p}\right. \\
& \left.+\frac{\epsilon}{2 N p K_{1} A_{0} r_{0}^{2}}\left(f\left(\xi^{2 q} \chi^{2 p^{\prime}+2} f^{p}\right)^{\frac{n}{n-2}}\right)^{\frac{n-2}{n}}\right] \\
\leq & 100 \epsilon \tilde{d}+C\left(\epsilon, n, p_{0}\right) p^{\frac{n}{2 p_{0}-n}+1} q^{2} A_{0}^{\frac{n^{2}+3 n}{2 p_{0}-n}} K_{1}^{\frac{2 p_{0}}{2 p_{0}-n}} r_{0}^{\frac{2 n}{2 p_{0}-n}} f_{B_{i} \cap \Omega} \xi^{2 q-2} \chi^{2 p^{\prime}+2} f^{p},
\end{aligned}
$$

where $\nVdash f \|_{p_{0}, B_{i} \cap \Omega}=\left(\frac{1}{V_{g(t)}\left(B_{i}\right)} \int_{B_{i} \cap \Omega} f^{p_{0}}\right)^{\frac{1}{p_{0}}}$.

Combining the above estimates, note that $\tilde{b} \leq \tilde{c}$. Then

$$
\begin{aligned}
\frac{\partial}{\partial t}\left(f \xi^{2 q} \chi^{2 p^{\prime}} f^{p}\right) \leq & p(1+4 \epsilon-p) f \tilde{b}^{2}+100 \epsilon \tilde{d} \\
+ & \frac{2}{\epsilon} 10^{6} n^{3} p^{3} q^{2}\left(f \tilde{a}^{2}+f \tilde{e}^{2}\right) \\
+ & C\left(\epsilon, n, p_{0}\right) p^{\frac{n}{2 p_{0}-n}+1} q^{2} A_{0}^{\frac{n^{2}+3 n}{2 p_{0}-n}} K_{1}^{\frac{2 p_{0}}{2 p_{0}-n}}\left(r_{0}^{\frac{2 n}{2 p_{0}-n}}+1\right) \\
& \cdot\left(f \xi^{2 q-2} \chi^{2 p^{\prime}+2} f^{p}\right) .
\end{aligned}
$$

On the other hand,

$$
\begin{aligned}
\left|\nabla\left(\xi^{q} \chi^{p^{\prime}+1} f^{\frac{p}{2}}\right)\right|^{2} & \leq\left(q \tilde{e}+\left(p^{\prime}+1\right) \tilde{a}+\frac{p}{2} \tilde{b}\right)^{2} \\
& \leq\left(2+\frac{1}{2 \epsilon}\right) q^{2} \tilde{e}^{2}+\left(2+\frac{1}{2 \epsilon}\right)\left(p^{\prime}+1\right)^{2} \tilde{a}^{2}+\left(\frac{1}{4}+\epsilon\right) p^{2} \tilde{b}^{2} .
\end{aligned}
$$

Then

$$
f \tilde{b}^{2} \geq\left[\left(\frac{1}{4}+\epsilon\right) p^{2}\right]^{-1} \tilde{d}-\left[\left(\frac{1}{4}+\epsilon\right) p^{2}\right]^{-1}\left(2+\frac{1}{2 \epsilon}\right)\left(q+p^{\prime}+1\right)^{2} f\left(\tilde{a}^{2}+\tilde{e}^{2}\right)
$$


By (5.12), (5.13), and the estimate $f \tilde{e}^{2}$, using $\left|\tilde{\nabla} \xi_{i}\right|^{2} \leq C\left(r_{0}, N\right)=\frac{8}{r_{0}^{2}}(N+1)$ as in Lemma 5.5, $0 \leq \xi_{i} \leq 1,0 \leq \chi \leq 1$, also noting that $1+4 \epsilon-p<0$,

$$
\begin{aligned}
& \frac{\partial}{\partial t}\left(f \xi^{2 q} \chi^{2 p^{\prime}} f^{p}\right)+\left(\frac{4(p-1-4 \epsilon)}{(1+4 \epsilon) p}-100 \epsilon\right) \tilde{d} \\
& \quad \leq C\left(\epsilon, n, p_{0}\right) p^{\frac{n}{2 p_{0}-n}+3} q^{2} A_{0}^{\frac{\left(2 p_{0}+3\right) n}{2 p_{0}-n}} K_{1}^{\frac{2 p_{0}}{2 p_{0}-n}}\left[r_{0}^{2}+r_{0}^{\frac{2 n}{2 p_{0}-n}}\right] f \xi^{2 q-2} \chi^{2 p^{\prime}} f^{p} .
\end{aligned}
$$

Choose $\epsilon=\frac{1}{1000}$ in (5.14). Then

$$
\begin{aligned}
& \frac{\partial}{\partial t}\left(f \xi^{2 q} \chi^{2 p^{\prime}} f^{p}\right)+\frac{1}{10}\left(f\left|\nabla\left(\xi^{q} \chi^{p^{\prime}+1} f^{\frac{p}{2}}\right)\right|^{2}\right) \\
& \quad \leq C\left(n, p_{0}\right) p^{\frac{n}{2 p_{0}-n}+3} q^{2} A_{0}^{\frac{\left(2 p_{0}+3\right) n}{2 p_{0}-n}} K_{1}^{\frac{2 p_{0}}{2 p_{0}-n}}\left[r_{0}^{2}+r_{0}^{\frac{2 n}{2 p_{0}-n}}\right] f \xi^{2 q-2} \chi^{2 p^{\prime}} f^{p} .
\end{aligned}
$$

Replacing $f$ with $|R m|$, the lemma is proved.

For simplicity, we use $C_{1}$ intead of $C_{1}\left(A_{0}, K_{1}, n, p_{0}, r_{0}\right)$ in the rest of this section; similarly for $C_{2}$, etc. In the next lemma, we use the energy estimate and modified Moser iteration to get the local $C^{0}$-estimate of $\left|\chi^{2} R m(g(t))\right|$.

Lemma 5.7. If $0<t<T_{\max }$, then there exists some positive constant

$$
C_{2}\left(A_{0}, K_{1}, n, p_{0}, r_{0}\right)=C\left(n, p_{0}\right) A_{0}^{\left(\frac{3}{2 p_{0}}+1\right) n}\left(r_{0}^{\frac{n}{p_{0}}} K_{1}\right)
$$

such that

$$
\left|\chi^{2} R m(g(t))\right| \leq C_{2}\left[C_{1}^{\frac{n+2}{2 p_{0}}} t^{\frac{1}{p_{0}}}+t^{-\frac{n}{2 p_{0}}}\right] .
$$

Proof. Let $f(x, t)=|R m(x)|_{g(t)}$. Given any $t_{1}$ and $t_{2}$ such that $0<t_{1}<t_{2}<T_{\max }$, set

$$
\psi(t)=\left\{\begin{aligned}
0, & 0 \leq t \leq t_{1} \\
\frac{t-t_{1}}{t_{2}-t_{1}}, & t_{1} \leq t \leq t_{2} \\
1, & t_{2} \leq t<T_{\max }
\end{aligned}\right.
$$

By Lemma 5.6

$$
\begin{aligned}
& \frac{\partial}{\partial t}\left(f \xi_{i}^{2 q} \chi^{2 p^{\prime}} f^{p} d V_{g(t)}\right)+\frac{1}{10}\left(f\left|\nabla\left(\xi_{i}^{q} \chi^{p^{\prime}+1} f^{\frac{p}{2}}\right)\right|^{2} d V_{g(t)}\right) \\
& \quad \leq C_{1} p^{\frac{n}{2 p_{0}-n}+3} q^{2}\left(f \xi_{i}^{2 q-2} \chi^{2 p^{\prime}} f^{p} d V_{g(t)}\right) .
\end{aligned}
$$

Multiply (5.18) by $\psi(t)$, and integrate it from 0 to $t_{0}$ with respect to $t$, where $t_{2} \leq t_{0}<T_{\max }$. Then

$$
\begin{aligned}
& f\left(\xi^{2 q} \chi^{2 p^{\prime}} f^{p}\right) d V_{g\left(t_{0}\right)}+\frac{1}{10} \int_{t_{2}}^{t_{0}} f\left|\nabla\left(\xi^{q} \chi^{p^{\prime}+1} f^{\frac{p}{2}}\right)\right|^{2} \\
& \leq C A_{0}^{\frac{\left(2 p_{0}+3\right) n}{2 p_{0}-n}} K_{1}^{\frac{2 p_{0}}{2 p_{0}-n}}\left[r_{0}^{2}+r_{0}^{\frac{2 n}{2 p_{0}-n}}\right] p^{\frac{n}{2 p_{0}-n}+3} q^{2}\left(1+\frac{1}{t_{2}-t_{1}}\right) \int_{t_{1}}^{t_{0}} \\
& \quad \cdot\left(f \xi^{2(q-1)} \chi^{2 p^{\prime}} f^{p}\right) .
\end{aligned}
$$

Note (5.19) is in fact valid for any $0<t_{1}<t_{2} \leq t_{0}<T_{\max }$.

For $0<t \leq t_{0}$, denote

$$
H\left(p, p^{\prime}, q, t\right)=\int_{t}^{t_{0}} f \xi^{2 q} \chi^{2 p^{\prime}} f^{p} .
$$


Then for any $0<\iota<\iota^{\prime} \leq t_{0}<T_{\max }$, using (5.6), (5.19) and the Hölder inequality,

$$
\begin{aligned}
& H\left(p\left(1+\frac{2}{n}\right), p^{\prime}\left(1+\frac{2}{n}\right)+1, q\left(1+\frac{2}{n}\right), \iota^{\prime}\right)=\int_{\iota^{\prime}}^{t_{0}} f\left(\xi^{2 q} \chi^{2 p^{\prime}} f^{p}\right)^{\frac{2}{n}}\left(\xi^{q} \chi^{p^{\prime}+1} f^{\frac{p}{2}}\right)^{2} \\
& \quad \leq \int_{\iota^{\prime}}^{t_{0}}\left[f\left(\xi^{2 q} \chi^{2 p^{\prime}} f^{p}\right)\right]^{\frac{2}{n}} \cdot 4 A_{0} r^{2}\left[f\left|\nabla\left(\xi^{q} \chi^{p^{\prime}+1} f^{\frac{p}{2}}\right)\right|^{2}\right] d t \\
& \quad \leq 40 A_{0} r_{0}^{2}\left[C_{1} p^{\frac{n}{2 p_{0}-n}+3} q^{2}+\frac{1}{\iota^{\prime}-\iota}\right]^{1+\frac{2}{n}} H\left(p, p^{\prime}, q-1, \iota\right)^{1+\frac{2}{n}} .
\end{aligned}
$$

Denote $\alpha=\frac{n}{2 p_{0}-n}+3, \nu=1+\frac{2}{n}, \eta=\nu^{n+2}$, fix $t \in\left(0, t_{0}\right)$, and set

$$
\begin{aligned}
& p_{k}=p_{0} \nu^{k}, \quad p_{k}^{\prime}=p_{k}-\frac{n}{2}, \quad p_{0}^{\prime}=p_{0}-\frac{n}{2}, \\
& q_{k}=n \nu^{k}-\left(\frac{n}{2}+1\right), \quad q_{0}=\frac{n}{2}-1, \quad \iota_{k}=t\left(1-\eta^{-k}\right), \\
& H_{k}=H\left(p_{k}, p_{k}^{\prime}, q_{k}, \iota_{k}\right), \quad \Phi_{k}=H_{k}^{\frac{1}{p_{k}}}, \\
& \Phi_{0}=\left(\int_{0}^{t_{0}} f \xi^{n-2} \chi^{2 p_{0}-n} f^{p_{0}}\right)^{\frac{1}{p_{0}}} .
\end{aligned}
$$

Then for any $k \geq 0$,

$$
\begin{aligned}
H_{k+1} & =H\left(p_{k+1}, p_{k+1}^{\prime}, q_{k+1}, \iota_{k+1}\right) \\
& =H\left(p_{k}\left(1+\frac{2}{n}\right), p_{k}^{\prime}\left(1+\frac{2}{n}\right)+1,\left(q_{k}+1\right)\left(1+\frac{2}{n}\right), \iota_{k+1}\right) \\
& \leq 40 A_{0} r_{0}^{2}\left[C_{1} p_{k}^{\alpha}\left(q_{k}+1\right)^{2}+\frac{1}{\iota_{k+1}-\iota_{k}}\right]^{\nu} \cdot H\left(p_{k}, p_{k}^{\prime}, q_{k}, \iota_{k}\right)^{\nu} \\
& \leq 40 A_{0} r_{0}^{2}\left[C_{1} p_{0}^{\alpha} n^{2} \nu^{(\alpha+2) k}+t^{-1} \frac{\eta}{\eta-1} \eta^{k}\right]^{\nu} H_{k}^{\nu} \\
& \leq C\left(n, p_{0}\right) A_{0} r_{0}^{2}\left[C_{1}+t^{-1}\right]^{\nu} \nu^{(\alpha+n+2) k \nu} H_{k}^{\nu} .
\end{aligned}
$$

Taking the $p_{k+1}$ root on both sides of the above inequality,

$$
\Phi_{k+1} \leq\left[C\left(n, p_{0}\right) A_{0} r_{0}^{2}\right]^{p_{0}^{-1} \nu^{-(k+1)}} \nu^{(\alpha+n+2) \frac{k \nu^{-k}}{p_{0}}}\left[C_{1}+t^{-1}\right]^{p_{0}^{-1} \nu^{-k}} \Phi_{k} .
$$

By induction,

$$
\Phi_{k+1} \leq\left[C\left(n, p_{0}\right) A_{0} r_{0}^{2}\right]^{p_{0}^{-1}\left(\sigma_{k+1}-1\right)} \nu^{(\alpha+n+2) \frac{\sigma_{k}^{\prime}}{p_{0}}}\left[C_{1}+t^{-1}\right]^{p_{0}^{-1} \sigma_{k}} \Phi_{0},
$$

where $\sigma_{k}=\sum_{i=0}^{k} \nu^{-i}$ and $\sigma_{k}^{\prime}=\sum_{i=0}^{k} i \nu^{-i}$; note that $\frac{\sigma_{k+1}-1}{p_{0}} \leq \frac{n}{2 p_{0}}$. Then

$$
\begin{aligned}
\left|\xi_{i}^{\frac{n}{p_{0}}} \chi^{2} f(x, t)\right| & \leq \lim _{k \rightarrow \infty} \Phi_{k+1} \\
& \leq C\left(n, p_{0}\right) A_{0}^{\frac{n}{2 p_{0}}}\left[C_{1}+t^{-1}\right]^{\frac{n+2}{2 p_{0}}} r_{0}^{\frac{n}{p_{0}}} \cdot\left(\int_{0}^{t_{0}} f_{B_{i} \cap \Omega} \xi_{i}^{n-2} \chi^{2 p_{0}-n} f^{p_{0}}\right)^{\frac{1}{p_{0}}} .
\end{aligned}
$$

Letting $t_{0} \rightarrow t$ in the above and using (5.8),

$$
\begin{aligned}
\left|\xi_{i}^{\frac{n}{p_{0}}} \chi^{2} f(x, t)\right| & \leq C\left(n, p_{0}\right) A_{0}^{\frac{n}{2 p_{0}}}\left[C_{1}+t^{-1}\right]^{\frac{n+2}{2 p_{0}}} r_{0}^{\frac{n}{p_{0}}}\left[(2 N)^{\frac{1}{p_{0}}} K_{1}\right] t^{\frac{1}{p_{0}}} \\
& \leq C\left(n, p_{0}\right) A_{0}^{\frac{3 n}{2 p_{0}}}\left(r_{0}^{\frac{n}{p_{0}}} K_{1}\right)\left[C_{1}^{\frac{n+2}{2 p_{0}}} t^{\frac{1}{p_{0}}}+t^{-\frac{n}{2 p_{0}}}\right] .
\end{aligned}
$$

Now for any $x \in \Omega$, we assume $x \in B_{i_{0}} \cap \Omega$. Note that $\left|\xi_{i}^{2} \chi^{2} f\right| \leq\left|\xi_{i}^{\frac{n}{p_{0}}} \chi^{2} f\right|$; then $\left|\chi^{2} f\right|(x, t)=\left|\sum_{j=1}^{N} \xi_{j}^{2} \chi^{2} f(x, t)\right| \leq C\left(n, p_{0}\right) A_{0}^{\left(\frac{3}{2 p_{0}}+1\right) n}\left(r_{0}^{\frac{n}{p_{0}}} K_{1}\right)\left[C_{1}^{\frac{n+2}{2 p_{0}}} t^{\frac{1}{p_{0}}}+t^{-\frac{n}{2 p_{0}}}\right]$.

By replacing $f$ with $|R m|$, we obtain our conclusion. 
Straightforward from Lemma 5.7, we have the following two corollaries.

Corollary 5.8. If $0<t<T_{\max }$, there exists some positive constant

$$
C_{3}\left(A_{0}, K_{1}, n, p_{0}, r_{0}\right)=C\left(n, p_{0}\right) A_{0}^{\left(\frac{3}{2 p_{0}}+1\right) n}\left(r_{0}^{\frac{n}{p_{0}}} K_{1}\right)
$$

such that

$$
\left|\chi^{2} R c(g(t))\right| \leq C_{3}\left(t^{-\frac{n}{2 p_{0}}}+C_{1}^{\frac{n+2}{2 p_{0}}} t^{\frac{1}{p_{0}}}\right) .
$$

Corollary 5.9. If $0 \leq t<T_{\max }$, there exists some positive constant $C$, which is independent of $t$, such that

$$
\left|\chi^{2} \operatorname{Rm}(g(t))\right| \leq C
$$

Choose $T_{1}>0$ such that

$$
\exp \left(C_{1} p_{0}^{\frac{n}{2 p_{0}-n}+3} N T_{1}\right)=\frac{3}{2},
$$

where $C_{1}$ is from Lemma 5.6 and $N$ is from Lemma 5.4. Then

$$
T_{1}=C\left(n, p_{0}\right) A_{0}^{-\frac{5 p_{0} n}{2 p_{0}-n}} K_{1}^{-\frac{2 p_{0}}{2 p_{0}-n}}\left[r^{-2}+r_{0}^{\frac{2 n}{2 p_{0}-n}}\right]^{-1} .
$$

Proposition 5.10. If $0 \leq t<T_{\max }<T_{1}$, then

$$
\left(\frac{1}{V_{g(t)}\left(B_{i}\right)} \int_{B_{i} \cap \Omega}|R m(g(t))|^{p_{0}} d V_{g(t)}\right)^{\frac{1}{p_{0}}} \leq\left(\frac{3}{2} N\right)^{\frac{1}{p_{0}}} K_{1}, \quad i=1,2,3, \cdots .
$$

Proof. Set $f(x, t) \doteqdot|R m(x)|_{g(t)}$. Let $q=1, p^{\prime}=0, p=p_{0}$ in Lemma 5.6, then

$$
\frac{\partial}{\partial t}\left(f_{\Omega} \xi_{i}^{2} f^{p_{0}} d V_{g(t)}\right) \leq C_{1} p_{0}^{\frac{n}{2 p_{0}-n}+3} f_{B_{i} \cap \Omega} f^{p_{0}} d V_{g(t)} .
$$

For $\Omega$, define $\operatorname{Cov}(\Omega)=\left\{B_{i} \mid B_{i} \cap \bar{\Omega} \neq \emptyset\right\}$ and note that $\operatorname{Cov}(\Omega)$ has only a finite number of elements. We define

$$
\phi(t)=\sup _{B_{i} \in \operatorname{Cov}(\Omega)}\left[f_{B_{i} \cap \Omega} \xi_{i}^{2}|R m|^{p_{0}} d V_{g(t)}\right] .
$$

Assume $\phi\left(t_{0}\right)=f_{B_{i_{0}} \cap \Omega} \xi_{i_{0}}^{2}|R m|^{p_{0}} d V_{g\left(t_{0}\right)}$; then

$$
\begin{aligned}
\frac{\partial}{\partial t} \phi\left(t_{0}\right) & \leq \frac{\partial}{\partial t}\left(f_{\Omega} \xi_{i_{0}}^{2} f^{p_{0}}\right)\left(t_{0}\right) \leq C_{1} p_{0}^{\frac{n}{2 p_{0}-n}+3} f_{B_{i_{0}} \cap \Omega} f^{p_{0}} d V_{g\left(t_{0}\right)} \\
& \leq C_{1} p_{0}^{\frac{n}{2 p_{0}-n}+3} \sum_{j=1}^{N}\left(\int_{B_{i_{0}} \cap \Omega} \xi_{j}^{2} f^{p_{0}} d V_{g\left(t_{0}\right)}\right) \leq C_{1} p_{0}^{\frac{n}{2 p_{0}-n}+3} N \phi\left(t_{0}\right) .
\end{aligned}
$$

Because $t_{0}$ is chosen freely,

$$
\frac{\partial}{\partial t} \phi(t) \leq\left[C_{1} p_{0}^{\frac{n}{2 p_{0}-n}+3} N\right] \phi(t) .
$$

By (5.1), $\phi(0) \leq \sup _{B_{i}} f_{B_{i} \cap \Omega}|R m|^{p_{0}} d V_{g_{0}} \leq K_{1}^{p_{0}}$. Then by the definition of $T_{1}$ and (5.24),

$$
\phi(t) \leq \frac{3}{2} K_{1}^{p_{0}}
$$

Now

$$
f_{B_{i} \cap \Omega}|R m|^{p_{0}} d V_{g(t)}=\sum_{j=1}^{N} f_{B_{i} \cap \Omega} \xi_{j}^{2} f^{p_{0}} \leq N \phi(t) \leq \frac{3}{2} N K_{1}^{p_{0}} .
$$


Define

$$
T_{1,1} \doteqdot\left[\frac{\left(2 p_{0}-n\right) \ln 2}{8 p_{0}(n+1)} C_{3}^{-1}\right]^{\frac{2 p_{0}}{2 p_{0}-n}}, \quad T_{1,2} \doteqdot\left[\frac{p_{0} \ln 2}{4\left(p_{0}+1\right)(n+1)} C_{1}^{-1} C_{3}^{-\left(\frac{n+2}{2 p_{0}}\right)}\right]^{\frac{p_{0}}{p_{0}+1}}
$$

and recall $T_{1}$ is defined in (5.22). Define

$$
T_{2} \doteqdot \min \left\{T_{1}, T_{1,1}, T_{1,2}\right\} \text {. }
$$

Proposition 5.11. If $0 \leq t<T_{\max }<T_{2}$, then

$$
2^{-\frac{1}{n+1}} g_{0} \leq g(t) \leq 2^{\frac{1}{n+1}} g_{0} \text {. }
$$

Proof. Choose $0 \leq t_{0}<T_{\max }<T_{2}$. Then by Corollary [5.8, we have

$$
\int_{0}^{t_{0}}\left|\frac{\partial}{\partial t} g\right| d t=\int_{0}^{t_{0}}\left|2 \chi^{2} R c(g(t))\right| d t \leq \int_{0}^{t_{0}} 2 C_{3}\left(t^{-\frac{n}{2 p_{0}}}+C_{1}^{\frac{n+2}{2 p_{0}}} t^{\frac{1}{p_{0}}}\right) d t \leq \frac{\ln 2}{n+1} .
$$

By Lemma 6.49 in [1], we get our conclusion.

Proposition 5.12. If $0 \leq t<T_{\max }<T_{2}$, then

$$
\left(f_{B_{x}\left(4 r_{0}\right)} h^{\frac{2 n}{n-2}} d V_{g(t)}\right)^{\frac{n-2}{n}} \leq 2 A_{0} r^{2} f_{B_{x}\left(4 r_{0}\right)}|\nabla h|^{2} d V_{g(t)}, \quad \forall h \in C_{0}^{\infty}\left(B_{x}\left(4 r_{0}\right)\right) .
$$

Proof. By Lemma 5.1 and Proposition 5.11, we get our conclusion.

Proposition 5.13. If $T_{\max }<T_{2}$, then on $\left[0, T_{\max }\right)$, we have

$$
\left\{\begin{aligned}
\left(f_{B_{x}\left(4 r_{0}\right)} h^{\frac{2 n}{n-2}} d V_{g(t)}\right)^{\frac{n-2}{n}} & \leq 2 A_{0} r^{2} f_{B_{x}\left(4 r_{0}\right)}|\nabla h|^{2} d V_{g(t)}, \\
2^{-\frac{1}{4}} g_{0} & \leq g(t) \leq 2^{\frac{1}{4}} g_{0}, \\
\left(f_{B_{i} \cap \Omega}|R m(g(t))|^{p_{0}} d V_{g(t)}\right)^{\frac{1}{p_{0}}} & \leq\left(\frac{3}{2} N\right)^{\frac{1}{p_{0}}} K_{1}, \\
\left|\chi^{2} R m(g(t))\right| & \leq C
\end{aligned}\right.
$$

for any $x \in M$ and $h \in C_{0}^{\infty}\left(B_{x}\left(4 r_{0}\right)\right)$, where $C$ is independent of $t$.

Proof. Combining Propositions 5.10, 5.11, 5.12 and Corollary 5.9, we get our conclusion.

Theorem 5.14. Assume $\left(M^{n}, g_{0}\right)$ is an $n$-dimensional $(n \geq 3)$ complete noncompact Riemannian manifold, which satisfies (5.1). Then the local Ricci flow (1.2) has a smooth solution on $\left[0, \frac{T_{2}}{2}\right]$, where $T_{2}$ is defined in (5.25). Moreover, for $t \in\left[0, \frac{T_{2}}{2}\right]$, the metric satisfies (5.7) and the curvature tensors satisfy the bounds (5.17) and (5.21).

Proof. First, by Theorem 2.9 and DeTurck's trick, equation (1.2) has a smooth solution on a sufficiently small time interval starting at $t=0$. Let $\left[0, T_{\max }\right)$ be a maximal time interval on which (1.2) has a smooth solution and such that (5.6), (5.7), (5.8) hold for each metric $g(t)$. We claim that $T_{\max }>\frac{T_{2}}{2}$; we prove it by contradiction.

If $T_{\max } \leq \frac{T_{2}}{2}$, then $T_{\max }<T_{2}$. By Proposition 5.13, $\left|\chi^{2} R m\right|_{(g(t), \infty)}$ is uniformly bounded on $\left[0, T_{\max }\right)$. Then by Theorem 4.8, we can extend the solution of local Ricci flow to $\left[0, T_{\max }+\delta\right)$, where $\delta>0$ is some constant. By Proposition 5.13, we can furthermore assume that (5.6), (5.7), (5.8) hold on $\left[0, T_{\max }+\delta\right)$, so it is in contradiction with the definition of $T_{\max }$. Then we have $T_{\max }>\frac{T_{2}}{2}$, and (1.2) has a smooth solution on $\left[0, \frac{T_{2}}{2}\right]$. We prove the first conclusion and $g(t)$ satisfies (5.7). 
Note that $\frac{T_{2}}{2}<T_{\max }$. Then by Lemma 5.7 and Corollary 5.8, we get (5.17) and (5.21).

Remark 5.15. In fact we have the following conclusion by the observation that the power ration between $K_{1}$ and $r_{0}$ is exactly $\frac{p_{0}}{n}$ in the local Ricci flows' existence time $\frac{T_{2}}{2}$ (in fact in $T_{1}, T_{1,1}$ and $T_{1,2}$ ).

Assume $\left(M^{n}, g_{0}\right)$ is an $n$-dimensional $(n \geq 3)$ complete noncompact Riemannian manifold, which satisfies

$$
\left\{\begin{array}{r}
R c \geq 0, \\
\lim _{r \rightarrow \infty} \frac{V\left(B_{x_{0}}(r)\right)}{r^{n}}=\delta>0, \\
\left(\int_{M^{n}}\left|R m\left(g_{0}\right)\right|^{p_{0}} d V_{g_{0}}\right)^{\frac{1}{p_{0}}} \leq C_{0}<\infty,
\end{array}\right.
$$

where $x_{0} \in M^{n}$ is a fixed point, $p_{0}>\frac{n}{2}$ and $C_{0}$ are positive constants.

Let $\Omega_{r} \doteqdot B_{x_{0}}(r), r \geq 1$, be a positive constant. Then the local Ricci flow (1.2) with repect to $\Omega_{r}$ has a smooth solution on $[0, T]$, where $T>0$ is independent of $r$.

The proof of the above conclusion is similar to Theorem [5.14, but not using $\left\{\xi_{i}\right\}_{i=1}^{\infty}$ in the whole procedure.

\section{Existence time estimates (The Second Case: Scale-Invariant EXPONENT)}

In this section, we assume that $\left(M^{n}, g_{0}\right)$ is an $n$-dimensional $(n \geq 3)$ complete noncompact Riemannian manifold satisfying the following assumptions:

$$
\left\{\begin{aligned}
V_{g_{0}}\left(B_{x}(\rho)\right) & \geq \widehat{N}_{1}\left(\frac{\rho}{4 r_{0}}\right)^{n} V_{g_{0}}\left(B_{x}\left(4 r_{0}\right)\right), \quad 0<\rho \leq 4 r_{0} \\
\left(\int_{B_{x}\left(r_{0}\right)} h^{\frac{2 n}{n-2}} d V_{g_{0}}\right)^{\frac{n-2}{n}} & \leq \widehat{A}_{0} \int_{B_{x}\left(r_{0}\right)}|\nabla h|^{2} d V_{g_{0}} \\
\left(\int_{B_{x}\left(r_{0}\right)}\left|R m\left(g_{0}\right)\right|^{\frac{n}{2}} d V_{g_{0}}\right)^{\frac{2}{n}} & \leq\left(\tau n \widehat{A}_{0}\right)^{-1} \\
\left(\int_{B_{x}\left(r_{0}\right)}\left|R c\left(g_{0}\right)\right|^{p_{0}} d V_{g_{0}}\right)^{\frac{1}{p_{0}}} & \leq \widehat{K}_{1}
\end{aligned}\right.
$$

for any $x \in M^{n}, h(x) \in C_{0}^{\infty}\left(B_{x}\left(r_{0}\right)\right)$, where $p_{0}>\frac{n}{2}, \widehat{A}_{0} \geq 1, \widehat{N}_{1} \leq 1, \widehat{K}_{1}$ and $r_{0}$ are positive constants, $\tau=300 \widehat{N} p_{0}$ and $\widehat{N}=2^{4 n} \widehat{N}_{1}^{-2} \geq 1$ as in Lemma 5.4, but using $\widehat{N}_{1}$, not $N_{1}$.

By the doubling property assumption in (6.1), we can choose $B_{i}$ as in Lemma 5.4. By an argument similar to the one in section 5 , we can assume that $\left[0, T_{\max }\right)$ is the maximal time interval on which the local Ricci flow (1.2) has a smooth solution and the following holds for each metric $g(t)$ (where $g(0)=g_{0}$ ):

$$
\begin{gathered}
\left(\int_{B_{x}\left(r_{0}\right)} h^{\frac{2 n}{n-2}} d V_{g(t)}\right)^{\frac{n-2}{n}} \leq 4 \widehat{A}_{0} \int_{B_{x}\left(r_{0}\right)}|\nabla h|^{2} d V_{g(t)}, \\
\frac{1}{2} g_{0} \leq g(t) \leq 2 g_{0} \\
\left(\int_{B_{i} \cap \Omega}|R m(g(t))|^{\frac{n}{2}} d V_{g(t)}\right)^{\frac{2}{n}} \leq(2 \widehat{N})^{\frac{2}{n}}\left(\tau n \widehat{A}_{0}\right)^{-1}, \quad i=1,2,3, \cdots,
\end{gathered}
$$

for any $x \in M^{n}, h \in C_{0}^{\infty}\left(B_{x}\left(r_{0}\right)\right)$. 
Lemma 6.1. When $0 \leq t<T_{\max }$, for any $i, q \geq 1, p \geq \frac{n}{2}, p^{\prime} \geq 0$ and $p \geq p^{\prime}$, there exists the constant $\widehat{C}_{1}\left(n, \widehat{N}_{1}, r_{0}\right)=C\left(n, \widehat{N}_{1}\right)\left(r_{0}^{-2}+1\right)>0$ such that

$$
\begin{aligned}
& \frac{\partial}{\partial t}\left(\int_{\Omega} \xi_{i}^{2 q} \chi^{2 p^{\prime}}|R m|^{p} d V_{g(t)}\right)+\theta\left(\int_{\Omega}\left|\nabla\left(\xi_{i}^{q} \chi^{p^{\prime}+1}|R m|^{\frac{p}{2}}\right)\right|^{2} d V_{g(t)}\right) \\
& \quad \leq \frac{1}{\epsilon} \widehat{C}_{1}\left(n, \widehat{N}_{1}, r_{0}\right) p^{3} q^{2}\left(\int_{B_{i} \cap \Omega} \xi_{i}^{2 q-2} \chi^{2 p^{\prime}}|R m|^{p} d V_{g(t)}\right),
\end{aligned}
$$

where

$$
\theta=\left(\frac{4(p-1-4 \epsilon)}{(1+4 \epsilon) p}\right)-48 p \widehat{A}_{0}|R m|_{\left(g(t), L^{\frac{n}{2}}\left(B_{i} \cap \Omega\right)\right)}
$$

and $|R m|_{\left(g(t), L^{\frac{n}{2}}\left(B_{i} \cap \Omega\right)\right)} \doteqdot\left(\int_{B_{i} \cap \Omega}|R m(x, t)|^{\frac{n}{2}} d V_{g(t)}\right)^{\frac{2}{n}}, \epsilon>0$, is any positive constant.

Proof. Let $f(x, t)=|R m(x, t)|_{g(t)}$ and

$$
\frac{\partial}{\partial t}\left(\int_{\Omega} \xi^{2 q} \chi^{2 p^{\prime}} f^{p}\right) \leq \sum_{k=1}^{6} I_{k}
$$

where

$$
\begin{aligned}
& I_{1}=p \int \xi^{2 q} \chi^{2 p^{\prime}+2} f^{p-1} \Delta f, \quad I_{2}=p \int \xi^{2 q} \chi^{2 p^{\prime}+2} f^{p-2}|\nabla f|^{2}, \\
& I_{3}=-(1-\epsilon) p \int \xi^{2 q} \chi^{2 p^{\prime}+2} f^{p-2}|\nabla R m|^{2}, \\
& I_{4}=10 p \int \xi^{2 q} \chi^{2 p^{\prime}+2} f^{p+1}-\int \xi^{2 q} \chi^{2 p^{\prime}+2} f^{p} R, \\
& I_{5}=\left(\frac{10^{5}}{\epsilon}\right) n p \int \xi^{2 q} \chi^{2 p^{\prime}}|\nabla \chi|^{2} f^{p}, \\
& I_{6}=8 p \int \xi^{2 q} \chi^{2 p^{\prime}+1} f^{p-2} g^{r i} g^{s j} g^{p k} g^{q l} R_{r s p q} \chi_{i l} R_{j k} .
\end{aligned}
$$

For simplification, we set the following notation:

$$
\begin{aligned}
& \tilde{a}=\xi^{q} \chi^{p^{\prime}} f^{\frac{p}{2}}|\nabla \chi|, \quad \tilde{b}=\xi^{q} \chi^{p^{\prime}+1} f^{\frac{p}{2}-1}|\nabla f|, \\
& \tilde{c}=\xi^{q} \chi^{p^{\prime}+1} f^{\frac{p}{2}-1}|\nabla R m|, \quad \tilde{d}=\int\left|\nabla\left(\xi^{q} \chi^{p^{\prime}+1} f^{\frac{p}{2}}\right)\right|^{2}, \quad \tilde{e}=\xi^{q-1} \chi^{p^{\prime}+1} f^{\frac{p}{2}}|\nabla \xi| .
\end{aligned}
$$

Note that $|R| \leq n|R m|=2 p f$ and

$$
\begin{aligned}
I_{4} & \leq 12 p \int \xi^{2 q} \chi^{2 p^{\prime}+2} f^{p+1} \leq 12 p|f|_{\left(g(t), L^{\frac{n}{2}}\left(B_{i} \cap \Omega\right)\right)} \cdot 4 \widehat{A}_{0} \cdot \int\left|\nabla\left(\xi^{q} \chi^{p^{\prime}+1} f^{\frac{p}{2}}\right)\right|^{2} \\
& \leq 48 p \widehat{A}_{0}|f|_{\left(g(t), L^{\frac{n}{2}}\left(B_{i} \cap \Omega\right)\right)} \cdot \tilde{d} .
\end{aligned}
$$

Similar to the proof of Lemma 5.1 .

$$
\begin{aligned}
\frac{\partial}{\partial t}\left(\int_{\Omega} \xi^{2 q} \chi^{2 p^{\prime}} f^{p}\right) \leq & p(1+4 \epsilon-p) \int \tilde{b}^{2}+48 p \widehat{A}_{0}\|f\|_{\frac{n}{2}, B_{i} \cap \Omega} \cdot \tilde{d} \\
& +\frac{2}{\epsilon} 10^{6} n^{3} p^{3} q^{2}\left(\int \tilde{a}^{2}+\int \tilde{e}^{2}\right) .
\end{aligned}
$$


Then by (5.13),

$$
\begin{aligned}
& \frac{\partial}{\partial t}\left(\int \xi^{2 q} \chi^{2 p^{\prime}} f^{p}\right)+\theta \tilde{d} \leq \frac{4}{\epsilon} 10^{6} n^{3} p^{3} q^{2} \int\left(\tilde{a}^{2}+\tilde{e}^{2}\right) \\
& \quad \leq \frac{8}{\epsilon} 10^{6} n^{3} p^{3} q^{2}\left(\int_{\Omega} \xi^{2 q} \chi^{2 p^{\prime}}|R m|^{p} d V_{g(t)}+\int_{\Omega} \xi^{2 q-2} \chi^{2 p^{\prime}+2}|R m|^{p}|\nabla \xi|^{2} d V_{g(t)}\right),
\end{aligned}
$$

where $\theta$ is defined in (6.6). Replace $f$ with $|R m|$; by Lemma $5.5,\left|\tilde{\nabla} \xi_{i}\right|^{2} \leq \frac{8}{r_{0}^{2}}(\widehat{N}+1)$, and the lemma is proved.

Lemma 6.2. When $0<t<T_{\max }$, for $i=1,2,3, \cdots$, there exists a constant $C\left(n, p_{0}, \widehat{N}_{1}\right)$ such that

$$
\int_{\Omega} \xi_{i}^{4} \chi^{2}|R m(x)|^{1+\frac{n}{2}} d V_{g(t)} \leq C\left(n, p_{0}, \widehat{N}_{1}\right)\left(r_{0}^{-2}+1\right)\left(\widehat{A}_{0}\right)^{-\frac{n}{2}}(t+1)^{2} t^{-1} .
$$

Proof. Let $f(x, t)=|R m(x)|_{g(t)}$. Using Lemma 6.1, letting $q=1, p^{\prime}=0, p=\frac{n}{2}$, $\epsilon=\frac{1}{16}$, and plugging the above values into (6.6), we get $\theta \geq \frac{1}{6}$. Then

$$
\begin{aligned}
& \frac{\partial}{\partial t}\left(\int_{\Omega} \xi^{2} f^{\frac{n}{2}} d V_{g(t)}\right)+\frac{1}{6} \int_{\Omega}\left|\nabla\left(\xi \chi f^{\frac{n}{4}}\right)\right|^{2} d V_{g(t)} \\
& \leq \frac{1}{\epsilon_{0}} C\left(n, \widehat{N}_{1}\right)\left(r_{0}^{-2}+1\right)\left(\int_{B_{i} \cap \Omega} f^{\frac{n}{2}} d V_{g(t)}\right) .
\end{aligned}
$$

Multiply the above by $\psi_{t_{1}, t_{2}}(t)$, which is defined in the proof of Lemma 5.7. Integrate from 0 to $t_{0}$ with respect to $t$, where $t_{0}$ satisfies $t_{2} \leq t_{0}<T_{\max }$. Then let $t_{1}=\frac{t_{0}}{4}, t_{2}=\frac{t_{0}}{2}$, and by (6.4),

$$
\begin{aligned}
\int_{\frac{t_{0}}{2}}^{t_{0}} \int_{\Omega}\left|\nabla\left(\xi \chi f^{\frac{n}{4}}\right)\right|^{2} & \leq C\left(n, \widehat{N}_{1}\right)\left(r_{0}^{-2}+1\right)\left(1+\frac{1}{t_{0}}\right) \int_{\frac{t_{0}}{4}}^{t_{0}} \int_{B_{i} \cap \Omega} f^{\frac{n}{2}} \\
& \leq C\left(n, p_{0}, \widehat{N}_{1}\right)\left(r_{0}^{-2}+1\right)\left(\widehat{A}_{0}\right)^{-\frac{n}{2}}\left(1+\frac{1}{t_{0}}\right) t_{0} .
\end{aligned}
$$

Again in Lemma 6.1, let $q=2, p^{\prime}=1, p=1+\frac{n}{2}$, and choose $\epsilon=\frac{1}{16}$. Then $\theta \geq \frac{1}{3}>0$ and

$$
\frac{\partial}{\partial t}\left(\int_{\Omega} \xi^{4} \chi^{2} f^{1+\frac{n}{2}} d V_{g(t)}\right) \leq C\left(n, \widehat{N}_{1}\right)\left(r_{0}^{-2}+1\right)\left(\int_{\Omega} \xi^{2} \chi^{2} f^{1+\frac{n}{2}} d V_{g(t)}\right) .
$$

Multiply the above with $\psi$, and integrate from 0 to $t_{0}$, where $t_{2} \leq t_{0}<T_{\max }$. Then let $t_{1}=\frac{t_{0}}{2}, t_{2}=t_{0}$, and from (6.2), (6.4) and (6.10),

$$
\begin{aligned}
\int_{\Omega} \xi^{4} \chi^{2} f^{1+\frac{n}{2}} d V_{g\left(t_{0}\right)} & \leq C\left(n, \widehat{N}_{1}\right)\left(r_{0}^{-2}+1\right)\left(1+\frac{1}{t_{0}}\right)\left(\int_{\frac{t_{0}}{2}}^{t_{0}} \int_{\Omega} \xi^{2} \chi^{2} f^{1+\frac{n}{2}}\right) \\
& \leq C\left(n, p_{0}, \widehat{N}_{1}\right)\left(r_{0}^{-2}+1\right)\left(\widehat{A}_{0}\right)^{-\frac{n}{2}}\left(1+\frac{1}{t_{0}}\right)^{2} t_{0} .
\end{aligned}
$$

Because we choose $t_{0}$ arbitrarily, for any $0<t<T_{\max }$,

$$
\int_{\Omega} \xi^{4} \chi^{2} f^{1+\frac{n}{2}} d V_{g(t)} \leq C\left(n, p_{0}, \widehat{N}_{1}\right)\left(r_{0}^{-2}+1\right)\left(\widehat{A}_{0}\right)^{-\frac{n}{2}}(t+1)^{2} t^{-1} .
$$

Replacing $f$ with $|R m|$, we get our conclusion. 
Lemma 6.3. When $0<t<T_{\max }, q \geq 2, p \geq \frac{n}{2}, p^{\prime} \geq 0$ and $p \geq p^{\prime}$, there exists a constant $C\left(n, p_{0}, \widehat{N}_{1}\right)$ such that

$$
\begin{aligned}
& \frac{\partial}{\partial t}\left(\int_{\Omega} \xi_{i}^{2 q} \chi^{2 p^{\prime}}|R c|^{p}\right)+\frac{1}{9}\left(\int_{\Omega}\left|\nabla\left(\xi_{i}^{q} \chi^{p^{\prime}+1}|R c|^{\frac{p}{2}}\right)\right|^{2}\right) \\
& \leq C\left(n, p_{0}, \widehat{N}_{1}\right) p^{2+\frac{n}{2}} q^{2}\left(r_{0}^{-2}+1\right)(t+1)^{2} t^{-1}\left(\int_{B_{i} \cap \Omega} \xi_{i}^{2(q-2)} \chi^{2 p^{\prime}}|R c|^{p}\right) .
\end{aligned}
$$

There also exists $\widehat{C}_{2}\left(n, \widehat{N}_{1}\right)>0$ such that

$$
\begin{aligned}
& \frac{\partial}{\partial t}\left(\int_{\Omega} \xi_{i}^{2} \chi^{2 p^{\prime}}|R c|^{p} d V_{g(t)}\right)+\theta\left(\int_{\Omega}\left|\nabla\left(\xi_{i} \chi^{p^{\prime}+1}|R c|^{\frac{p}{2}}\right)\right|^{2} d V_{g(t)}\right) \\
& \quad \leq \frac{1}{\epsilon} \widehat{C}_{2}\left(n, \widehat{N}_{2}\right) p^{3}\left(r_{0}^{-2}+1\right)\left(\int_{B_{i} \cap \Omega} \chi^{2 p^{\prime}}|R c|^{p}\right),
\end{aligned}
$$

where $\theta=\left[\frac{4(p-1-4 \epsilon)}{(1+4 \epsilon) p}-\frac{32 p \widehat{N} \frac{2}{n}}{\tau \sqrt{n}}\right]$ and $\epsilon>0$ is any positive constant.

Proof. Let $f(x, t)=|R c(x)|_{g(t)}$. By Lemma 3.4

$$
\frac{\partial}{\partial t}\left(\int_{\Omega} \xi^{2 q} \chi^{2 p^{\prime}} f^{p}\right) \leq \sum_{k=1}^{6} I_{k}
$$

where

$$
\begin{aligned}
& I_{1}=p \int \xi^{2 q} \chi^{2 p^{\prime}+2} f^{p-1} \Delta f, \quad I_{2}=p \int \xi^{2 q} \chi^{2 p^{\prime}+2} f^{p-2}|\nabla f|^{2}, \\
& I_{3}=-(1-\epsilon) p \int \xi^{2 q} \chi^{2 p^{\prime}+2} f^{p-2}|\nabla R c|^{2}, \quad I_{4}=2(1+\sqrt{n}) p \int \xi^{2 q} \chi^{2 p^{\prime}+2} f^{p}|R m|, \\
& I_{5}=\left(\frac{10^{5}}{\epsilon}\right) n p \int \xi^{2 q} \chi^{2 p^{\prime}}|\nabla \chi|^{2} f^{p}, \quad I_{6}=p \int \xi^{2 q} \chi^{2 p^{\prime}} g^{i k} g^{j l} R_{k l} J_{2},
\end{aligned}
$$

where $J_{2}$ in $I_{6}$ is from (3.13).

Similar to the proof of Lemma 1.1, we set

$$
\begin{aligned}
& \tilde{a}=\xi^{q} \chi^{p^{\prime}} f^{\frac{p}{2}}|\nabla \chi|, \quad \tilde{b}=\xi^{q} \chi^{p^{\prime}+1} f^{\frac{p}{2}-1}|\nabla f|, \\
& \tilde{c}=\xi^{q} \chi^{p^{\prime}+1} f^{\frac{p}{2}-1}|\nabla R c|, \quad \tilde{d}=\int\left|\nabla\left(\xi^{q} \chi^{p^{\prime}+1} f^{\frac{p}{2}}\right)\right|^{2}, \quad \tilde{e}=\xi^{q-1} \chi^{p^{\prime}+1} f^{\frac{p}{2}}|\nabla \xi| .
\end{aligned}
$$

Then

$$
I_{1}+I_{2}+I_{3}+I_{5}+I_{6} \leq \frac{4(1+4 \epsilon-p)}{(1+4 \epsilon) p} \tilde{d}+\frac{4}{\epsilon} 10^{6} n^{3} p^{3} q^{2}\left(\int \tilde{a}^{2}+\int \tilde{e}^{2}\right) .
$$

Finally for $I_{4}$, when $q \geq 2$,

$$
\begin{aligned}
I_{4} \leq 2(1+\sqrt{n}) p\left(\int_{\Omega} \xi^{4} \chi^{2}|R m|^{1+\frac{n}{2}}\right)^{\frac{2}{n+2}} \cdot\left(\int_{B_{i} \cap \Omega} \xi^{2(q-2)} \xi^{2 p^{\prime}} f^{p}\right)^{\frac{2}{n+2}} \\
\quad \cdot\left[\int_{\Omega}\left(\xi^{q} \chi^{p^{\prime}+1} f^{\frac{p}{2}}\right)^{\frac{2 n}{n-2}}\right]^{\frac{n-2}{n+2}} \\
\leq \hat{a} \cdot \hat{b} .
\end{aligned}
$$


In the last inequality we used Lemma 6.2 and the following notation:

$$
\begin{aligned}
& \hat{a}=C\left(n, p_{0}, \widehat{N}_{1}\right) p\left[\left(r_{0}^{-2}+1\right)(t+1)^{2} t^{-1}\right]^{\frac{2}{n+2}}\left(\int_{B_{i} \cap \Omega} \xi^{2(q-2)} \chi^{2 p^{\prime}} f^{p}\right)^{\frac{2}{n+2}}, \\
& \hat{b}=\left(\int_{\Omega}\left|\nabla\left(\xi^{q} \chi^{p^{\prime}+1} f^{\frac{p}{2}}\right)\right|^{2}\right)^{\frac{n}{n+2}}, \\
& s=\frac{n+2}{2}, \quad s^{\prime}=\frac{n+2}{n}, \quad \frac{1}{s}+\frac{1}{s^{\prime}}=1 .
\end{aligned}
$$

For any $\delta>0$,

$$
\hat{a} \hat{b} \leq \frac{1}{s}\left(\hat{a} \delta^{-\frac{1}{s s^{\prime}}}\right)^{s}+\frac{1}{s^{\prime}}\left(\hat{b} \delta \frac{1}{s s^{\prime}}\right)^{s^{\prime}} \leq \hat{a}^{s} \delta^{-\frac{1}{s^{\prime}}}+\hat{b}^{s^{\prime}} \delta^{\frac{1}{s}} .
$$

Let $\delta=k_{0}^{\frac{n+2}{2}}$, where $k_{0}$ is some positive constant which will be determined later. Then

$$
I_{4} \leq k_{0}^{-\frac{n}{2}} C\left(n, p_{0}, \widehat{N}_{1}\right) p^{\frac{n+2}{2}}\left[\left(r_{0}^{-2}+1\right)(t+1)^{2} t^{-1}\right]\left(\int_{B_{i} \cap \Omega} \xi^{2(q-2)} \chi^{2 p^{\prime}} f^{p}\right)+k_{0} \tilde{d} .
$$

Now we choose $\epsilon=\frac{1}{16}, k_{0}=\frac{1}{9}$ in (6.14) and (6.15), and from (6.13),

$$
\begin{aligned}
& \frac{\partial}{\partial t}\left(\int_{\Omega} \xi^{2 q} \chi^{2 p^{\prime}} f^{p}\right)+\frac{1}{3} \int_{\Omega}\left|\nabla\left(\xi^{q} \chi^{p^{\prime}+1} f^{\frac{p}{2}}\right)\right|^{2} \\
& \leq C\left(n, p_{0}, \widehat{N}_{1}\right) p^{2+\frac{n}{2}} q^{2}\left(r_{0}^{-2}+1\right)(t+1)^{2} t^{-1}\left(\int_{B_{i} \cap \Omega} \xi^{2(q-2)} \chi^{2 p^{\prime}} f^{p}\right) .
\end{aligned}
$$

Hence (6.11) is proved.

When $q=1$, from (6.2), (6.4) and the Hölder inequality,

$$
I_{4} \leq 8(1+\sqrt{n}) p \widehat{A}_{0}|R m|_{\left(g(t), L^{\frac{n}{2}}\left(B_{i} \cap \Omega\right)\right)} \cdot \tilde{d} \leq \frac{32 p \widehat{N}^{\frac{2}{n}}}{\tau \sqrt{n}} \tilde{d} .
$$

From (6.13), 6.14) and (6.16),

$$
\begin{aligned}
& \frac{\partial}{\partial t}\left(\int_{\Omega} \xi_{i}^{2} \chi^{2 p^{\prime}} f^{p} d V_{g(t)}\right)+\theta\left(\int_{\Omega}\left|\nabla\left(\xi_{i} \chi^{p^{\prime}+1} f^{\frac{p}{2}}\right)\right|^{2} d V_{g(t)}\right) \\
& \leq \frac{4}{\epsilon} 10^{6} n^{3} p^{3}\left(|\nabla \chi|_{\infty}^{2} \int_{\Omega} \xi_{i}^{2} \chi^{2 p^{\prime}} f^{p}+\int_{B_{i} \cap \Omega} \chi^{2 p^{\prime}+2} f^{p}\left|\nabla \xi_{i}\right|^{2}\right) .
\end{aligned}
$$

Replacing $f$ by $|R c|$ and simplifying it, we get (6.12).

Choose $\widehat{T}_{1}>0$ such that

$$
\exp \left(16 \widehat{C}_{2} p_{0}^{3} \widehat{N}\left(r_{0}^{-2}+1\right) \widehat{T}_{1}\right)=\frac{3}{2} .
$$

Corollary 6.4. When $0 \leq t<T_{\max } \leq \widehat{T}_{1}$,

$$
\left(\int_{B_{i} \cap \Omega}|R c|^{p_{0}} d V_{g(t)}\right)^{\frac{1}{p_{0}}} \leq\left(\frac{3}{2} \widehat{N}\right)^{\frac{1}{p_{0}}} \widehat{K}_{1}, \quad i=1,2,3, \cdots .
$$

Proof. Set $f=|R c|$ and choose $p^{\prime}=0, p=p_{0}$ and $\epsilon=\frac{1}{16}$ in (6.12). Then it is easy to check that $\theta \geq \frac{1}{15} \geq 0$ from the definition of $\theta$ in Lemma 6.3. Then

$$
\frac{\partial}{\partial t} \int_{\Omega} \xi_{i}^{2}|R c|^{p_{0}} \leq 16 \widehat{C}_{2} p_{0}^{3}\left(r_{0}^{-2}+1\right)\left(\int_{B_{i} \cap \Omega}|R c|^{p_{0}}\right) .
$$


Define $\phi(t)=\sup _{B_{i} \in \operatorname{Cov}(\Omega)} \int_{B_{i} \cap \Omega} \xi_{i}^{2}|R c|^{p_{0}} d V_{g(t)}$, and

$$
\frac{\partial}{\partial t} \phi(t) \leq 16 \widehat{C}_{2} p_{0}^{3}\left(r_{0}^{-2}+1\right) \widehat{N} \phi(t) .
$$

From the last inequality in (6.1),

$$
\phi(t) \leq \exp \left(16 \widehat{C}_{2} p_{0}^{3} \widehat{N}\left(r_{0}^{-2}+1\right) t\right) \phi(0) \leq \frac{3}{2} \widehat{K}_{1}^{p_{0}} .
$$

Now we have

$$
\int_{B_{i} \cap \Omega}|R c|^{p_{0}} d V_{g(t)} \leq \widehat{N} \phi(t) \leq\left(\frac{3}{2} \widehat{N}\right) \widehat{K}_{1}^{p_{0}}
$$

The conclusion is proved.

We still use Moser iteration to get the local $C^{0}$-estimate $\left|\chi^{2} R c(g(t))\right|$.

Proposition 6.5. If $0<t<T_{\max } \leq \widehat{T}_{1}$, there exists some constant $\widehat{C}_{3}\left(n, \widehat{N}_{1}, p_{0}\right)>$ 0 such that

$$
\left|\chi^{2} R c(g(t))\right| \leq \widehat{C}_{3}\left[\left(r_{0}^{-2}+1\right) \widehat{A}_{0}\right]^{\frac{n}{2 p_{0}}} \widehat{K}_{1}\left(t^{-\frac{n}{2 p_{0}}}+t^{\frac{n}{2 p_{0}}}\right) .
$$

Proof. Let $f(x, t)=|R c(x)|_{g(t)}$. From (6.11), use a similar argument as in Lemma 5.7. except define $H$ as the following:

$$
H\left(p, p^{\prime}, q, t\right)=\int_{t}^{t_{0}} \int_{B_{i} \cap \Omega} \xi^{2 q} \chi^{2 p^{\prime}} f^{p} .
$$

Denote $\nu=1+\frac{2}{n}, \eta=\nu^{n+2}$ and fix $t \in\left(0, t_{0}\right)$; set

$$
\begin{aligned}
& p_{k}=p_{0} \nu^{k}, \quad p_{k}^{\prime}=p_{k}-\frac{n}{2}, \\
& q_{k}=2 n \nu^{k}-(n+2), \quad q_{0}=n-2, \quad \tau_{k}=t\left(1-\eta^{-k}\right), \\
& H_{k}=H\left(p_{k}, p_{k}^{\prime}, q_{k}, \tau_{k}\right), \quad \Phi_{k}=H_{k}^{\frac{1}{p_{k}}}
\end{aligned}
$$

Then

$$
\Phi_{0}=\left(\int_{0}^{t_{0}} \int_{\Omega} \xi^{2(n-2)} \chi^{2 p_{0}-n} f^{p_{0}}\right)^{\frac{1}{p_{0}}}
$$

We can get

$$
\begin{aligned}
& \left|\xi_{i}^{\frac{2 n}{p_{0}}} \chi^{2} f(x, t)\right| \leq \lim _{k \rightarrow \infty} \Phi_{k+1} \\
& \leq C\left(n, \widehat{N}_{1}, p_{0}\right)\left(r_{0}^{-2}+1\right)^{\frac{n}{2 p_{0}}}\left(t_{0}+1\right)^{\frac{n}{p_{0}}} \widehat{A}_{0}^{\frac{n}{2 p_{0}}} t^{-\frac{n+2}{2 p_{0}}}\left(\int_{0}^{t_{0}} \int_{B_{i} \cap \Omega} \xi_{i}^{2(n-2)} \chi^{2 p_{0}-n} f^{p_{0}}\right)^{\frac{1}{p_{0}}} .
\end{aligned}
$$

Letting $t_{0} \rightarrow t$ in the above and using Corollary 6.4

$$
\left|\xi_{i}^{\frac{2 n}{p_{0}}} \chi^{2} f(x, t)\right| \leq C\left(n, \widehat{N}_{1}, p_{0}\right)\left[\left(r_{0}^{-2}+1\right) \widehat{A}_{0}\right]^{\frac{n}{2 p_{0}}} \widehat{K}_{1}\left(t^{-\frac{n}{2 p_{0}}}+t^{\frac{n}{2 p_{0}}}\right) .
$$


For any $x \in \Omega$, one can assume $x \in B_{i_{0}} \cap \Omega$, and note that $\left|\xi_{i}^{4} \chi^{2} f\right| \leq\left|\xi_{i}^{\frac{2 n}{p_{0}}} \chi^{2} f\right|$. Then

$$
\begin{aligned}
\left|\chi^{2} f\right|(x, t) & =\left|\sum_{j=1}^{\widehat{N}} \xi_{j}^{2} \chi^{2} f(x, t)\right|=\left|\left(\sum_{j=1}^{\widehat{N}} \xi_{j}^{2}\right) \chi^{2} f\right| \\
& \leq \widehat{C}\left(n, \widehat{N}_{1}, p_{0}\right)\left[\left(r_{0}^{-2}+1\right) \widehat{A}_{0}\right]^{\frac{n}{2 p_{0}}} \widehat{K}_{1}\left(t^{-\frac{n}{2 p_{0}}}+t^{\frac{n}{2 p_{0}}}\right) .
\end{aligned}
$$

Replacing $f$ with $|R c|$, we obtain our conclusion.

Lemma 6.6. When $0<t<T_{\max }$, for $q>2, p \geq \frac{n}{2}, p^{\prime} \geq 0$ and $p \geq p^{\prime}$, there exists a constant $C\left(n, p_{0}, \widehat{N}_{1}\right)$ such that

$$
\begin{aligned}
& \frac{\partial}{\partial t}\left(\int \xi_{i}^{2 q} \chi^{2 p^{\prime}}|R m|^{p}\right)+\frac{1}{9}\left(\int\left|\nabla\left(\xi_{i}^{q} \chi^{p^{\prime}+1}|R m|^{\frac{p}{2}}\right)\right|^{2}\right) \\
& \leq C\left(n, p_{0}, \widehat{N}_{1}\right) p^{2+\frac{n}{2}} q^{2}\left(r_{0}^{-2}+1\right)(t+1)^{2} t^{-1}\left(\int_{B_{i} \cap \Omega} \xi_{i}^{2(q-2)} \chi^{2 p^{\prime}}|R m|^{p}\right) .
\end{aligned}
$$

Proof. Using Lemma 6.2, the proof is similar to the proof of (6.11) in Lemma 6.3.

Lemma 6.7. If $0<t<T_{\max }$, there exists some positive constant $\widehat{C}_{4}\left(n, \widehat{N}_{1}, p_{0}\right)$ such that

$$
\left|\chi^{2} R m(g(t))\right| \leq \widehat{C}_{4}\left(r_{0}^{-2}+1\right)\left(t^{-1}+t\right) .
$$

Proof. Similar to the proof of Proposition 6.5, but replacing $p_{0}$ with $\frac{n}{2}$ and using Lemma 6.6 and (6.4) instead of Lemma 6.3 and Corollary 6.4.

Corollary 6.8. If $0<t<T_{\max }$, then there exists some positive constant $C$ independent of $t$ such that

$$
\left|\chi^{2} \operatorname{Rm}(g(t))\right| \leq C .
$$

Proof. Straightforward from Lemma 6.7

Choose $\widehat{T}_{2}>0$ such that

$$
\exp \left(2 n^{3} \widehat{C}_{1} \widehat{N} \widehat{T}_{2}\right)=\frac{3}{2}
$$

and choose $q \geq 1, p^{\prime} \geq 0, p \geq \frac{n}{2}, \epsilon \geq \frac{1}{16}$ in Lemma 6.1, and apply a similar argument as shown in Corollary 6.4 if $0 \leq t \leq T_{\max } \leq \widehat{T}_{2}$,

$$
\left(\int_{B_{i} \cap \Omega}|R m(g(t))|^{\frac{n}{2}} d V_{g(t)}\right)^{\frac{2}{n}} \leq\left(\frac{3}{2} \widehat{N}\right)^{\frac{2}{n}}\left(\tau n \widehat{A}_{0}\right)^{-1}, \quad i=1,2,3, \cdots
$$

Define

$$
\begin{aligned}
\widehat{T}_{3,1} & =\left[\frac{\left(2 p_{0}-n\right) \ln 2}{32 p_{0}} \widehat{C}_{3}^{-1} \widehat{K}_{1}^{-1}\left[\left(r_{0}^{-2}+1\right) \widehat{A}_{0}\right]^{-\frac{n}{2 p_{0}}}\right]^{\frac{2 p_{0}}{2 p_{0}-n}}, \\
\widehat{T}_{3,2} & =\left[\frac{\left(2 p_{0}+n\right) \ln 2}{32 p_{0}} \widehat{C}_{3}^{-1} \widehat{K}_{1}^{-1}\left[\left(r_{0}^{-2}+1\right) \widehat{A}_{0}\right]^{-\frac{n}{2 p_{0}}}\right]^{\frac{2 p_{0}}{2 p_{0}+n}}, \\
\widehat{T}_{3} & =\min \left\{\widehat{T}_{3,1}, \widehat{T}_{3,2}\right\} .
\end{aligned}
$$

By Proposition 6.5 and a similar argument as in Proposition [5.11] if $0 \leq t<$ $T_{\max } \leq \min \left\{\widehat{T}_{1}, \widehat{T}_{3}\right\}$, then $2^{-\frac{1}{4}} g_{0} \leq g(t) \leq 2^{\frac{1}{4}} g_{0}$. 
We define

$$
\widehat{T}_{4}=\min \left\{\widehat{T}_{1}, \widehat{T}_{2}, \widehat{T}_{3}\right\} .
$$

By the above argument, similar to Proposition 5.13, we have

Proposition 6.9. If $0<T_{\max } \leq \widehat{T}_{4}$, then on $\left[0, T_{\max }\right)$, we have

$$
\left\{\begin{aligned}
\left(\int_{B_{x}\left(r_{0}\right)} h^{\frac{2 n}{n-2}} d V_{g_{0}}\right)^{\frac{n-2}{n}} & \leq 2 \widehat{A}_{0} \int_{B_{x}\left(r_{0}\right)}|\nabla h|^{2} d V_{g_{0}}, \\
2^{-\frac{1}{4}} g_{0} \leq g(t) \leq 2^{\frac{1}{4}} g_{0}, & \\
\left(\int_{B_{i} \cap \Omega}|R m(g(t))|^{\frac{n}{2}} d V_{g(t)}\right)^{\frac{2}{n}} & \leq\left(\frac{3}{2} \widehat{N}\right)^{\frac{2}{n}}\left(\tau n \widehat{A}_{0}\right)^{-1}, \\
\left|\chi^{2} \operatorname{Rm}(g(t))\right| & \leq C
\end{aligned}\right.
$$

for any $x \in M^{n}$ and $h \in C_{0}^{\infty}\left(B_{x}\left(r_{0}\right)\right)$, where $C$ is independent of $t$.

By a similar argument to the proof of Theorem 5.14, we have the following theorem:

Theorem 6.10. Assume $\left(M^{n}, g_{0}\right)$ is an $n$-dimensional $(n \geq 3)$ complete noncompact Riemannian manifold, which satisfies (6.1). Then the local Ricci flow (1.2) has a smooth solution on $\left[0, \frac{\widehat{T}_{4}}{2}\right]$. Moreover, for $t \in\left(0, \frac{\widehat{T}_{4}}{2}\right]$, the metric satisfies (6.3) and the curvature tensors satisfy the estimates (6.19), (6.20).

\section{Short-time EXISTence of the Ricci flow}

In this section we consider the family of local Ricci flows converging to the Ricci flow on a noncompact manifold $M^{n}$. We can get the short-time existence of the Ricci flow on $M^{n}$ when we have the uniform lower bound of existence-time for the family of the local Ricci flows. At many points in this section, we will take a subsequence; to simplify notation, at each stage a sequence such as $\left\{\chi_{k}\right\}$ will be re-indexed to continue to be $\left\{\chi_{k}\right\}$.

Proof of Theorem 1.1, Choose

$$
\Omega_{i}=\left\{x \mid d_{g_{0}}(x, \mathbf{O}) \leq 4^{i}, x \in M\right\}, \quad i=1,2, \cdots,
$$

where $\mathbf{O}$ is some fixed point in $M$. Construct $\chi_{i}$, which is a smooth cut-off function on $M$, such that

$$
\chi_{i+1}(x)= \begin{cases}1, & x \in \Omega_{i} \\ 0, & x \in M \backslash \Omega_{i+1}\end{cases}
$$

and

$$
0 \leq \chi_{i} \leq 1, \quad\left\|\nabla \chi_{i}\right\|_{\infty} \leq 1, \quad \lim _{i \rightarrow \infty} \chi_{i}(x)=1 .
$$

Note that $T_{2}$ is independent of $\chi_{i}$. By Theorem 5.14 for each $k$, there exists $g_{k}(t)$ which is the solution of the following local Ricci flow on $\left[0, \frac{T_{2}}{2}\right]$ :

$$
\left\{\begin{aligned}
\frac{\partial}{\partial t} g_{k} & =-2 \chi_{k}^{2} R c, \quad x \in M \\
g_{k}(x, 0) & =g_{0}(x), \quad x \in M
\end{aligned}\right.
$$

Then fix $i$. For any $0<t_{1}<\frac{T_{2}}{2}$, we have

$$
\left\|\nabla_{k}^{m} R m_{k}\left(x, t_{1}\right)\right\|_{\left(g_{k}\left(t_{1}\right), \infty\right)} \leq C\left(m, \Omega_{i}, t_{1}\right), \quad x \in \Omega_{i}, \quad k \leq i,
$$

where $\nabla_{k}$ and $R m_{k}$ are with respect to $g_{k}(t)$ and $C_{(m)}^{i}$ is a sequence of constants independent of $k$. For simplicity, we use the notation $\left|\nabla_{k}^{m} R m_{k}\left(x, t_{1}\right)\right|_{\infty}$ instead of 
$\left\|\nabla_{k}^{m} R m_{k}\left(x, t_{1}\right)\right\|_{\left(g_{k}\left(t_{1}\right), \infty\right)}$. If $k>i$ on $\Omega_{i}, g_{k}$ satisfies (1.1), which is the Ricci flow. From (5.17)

$$
\left|\chi_{k}^{2} R m_{k}\left(x, t_{1}\right)\right|_{\infty} \leq C_{2}\left(t_{1}^{-\frac{n}{2 p_{0}}}+C_{1}^{\frac{n+2}{2 p_{0}}} t^{\frac{1}{p_{0}}}\right), \quad x \in M, \quad \forall k .
$$

Note that $C_{1}, C_{2}$ are independent of $k$. Hence by the definition of $\chi_{i}$,

$$
\left|R m_{k}\left(x, t_{1}\right)\right|_{\infty} \leq C_{2}\left(t_{1}^{-\frac{n}{2 p_{0}}}+C_{1}^{\frac{n+2}{2 p_{0}}} t^{\frac{1}{p_{0}}}\right), \quad x \in \Omega_{i}, k>i .
$$

Then by Theorem 14.14 in [3],

$$
\begin{aligned}
\left|\nabla_{k}^{m} R m_{k}\left(x, t_{1}\right)\right|_{\infty} \leq & C\left(A_{0}, K_{1}, n, m, p_{0}, r_{0}, t_{1}, \Omega_{i}\right) t_{1}^{-\frac{m}{2}} \\
& =C\left(A_{0}, K_{1}, n, m, p_{0}, r_{0}, t_{1}, \Omega_{i}\right)
\end{aligned}
$$

for any $x \in \Omega_{i}$ and $k>i$.

By (7.3) and (7.6),

$$
\left|\nabla_{k}^{m} R m_{k}\left(x, t_{1}\right)\right|_{\infty} \leq C_{(m)}^{i} \quad x \in \Omega_{i}, \forall k \geq 0,
$$

where $C_{(m)}^{i}$ is a sequence of constants independent of $k$.

When $t \in\left[0, \frac{T_{2}}{2}\right]$, by Theorem 5.14

$$
\frac{1}{2} g_{0} \leq g_{k}(t) \leq 2 g_{0}
$$

then

$$
C_{1} \sqrt{\operatorname{det} g_{0}} \leq \sqrt{\operatorname{det} g_{k}\left(t_{1}\right)} \leq C_{2} \sqrt{\operatorname{det} g_{0}} .
$$

By Lemma 5.2 of [15], there exist some $\tau_{0}>0$ such that

$$
i n j_{g_{k}\left(t_{1}\right)}(\mathbf{O}) \geq \tau_{0} .
$$

By (7.7) and (7.9), Theorem 3.9 in [2] applies to $\left(\Omega_{i}, g_{k}\left(t_{1}\right), \mathbf{O}\right)_{k \in \mathbb{N}}$. Then

$$
\lim _{k \rightarrow \infty} g_{k}\left(x, t_{1}\right)=g_{\infty}\left(x, t_{1}\right),
$$

where the limit is in $C^{\infty}$ sense on $\Omega_{i}$. Hence

$$
\left|\widehat{\nabla}^{m} g_{k}\left(x, t_{1}\right)\right|_{\infty} \triangleq\left|\nabla_{g_{0}}^{m} g_{k}\left(x, t_{1}\right)\right|_{\infty} \leq C_{(m)}^{i}, \quad x \in \Omega_{i},
$$

where $C_{(m)}^{i}$ is a sequence of constants independent of $k$.

From Theorem 14.14 in 3 and the argument for obtaining (7.6), when $k>i$, we have

$$
\left|\nabla_{k}^{m} R m_{k}(x, t)\right|_{\infty} \leq C_{(m)}^{i}, \quad(x, t) \in \Omega_{i} \times\left[t_{1}, \frac{T_{2}}{2}\right] .
$$

If $k \leq i$, it is easy to see that (7.11) is also true. So for any $k$, (7.11) is true, where $C_{(m)}^{i}$ is independent of $k$.

Then by (7.7), (7.10), (7.11) and Lemma 3.11 in [2],

$$
\left|\frac{\partial^{q}}{\partial t^{q}} \widehat{\nabla}^{m} g_{k}(x, t)\right|_{\infty} \leq C_{(m, q)}^{i}, \quad(x, t) \in \Omega_{i} \times\left[t_{1}, \frac{T_{2}}{2}\right], \quad \forall k \geq 0,
$$

where $C_{(m, q)}^{i}$ is a sequence of constants independent of $k$.

Now by the Arzela-Ascoli theorem, we get

$$
\lim _{k \rightarrow \infty} g_{k}(x, t)=g_{\infty}(x, t), \quad(x, t) \in \Omega_{i} \times\left[t_{1}, \frac{T_{2}}{2}\right],
$$


where the limit is in the $C^{\infty}$ sense. Because $i$ and $t_{1}$ are arbitrary, by the diagonal method,

$$
g(x, t) \triangleq g_{\infty}(x, t)=\lim _{k \rightarrow \infty} g_{k}(x, t), \quad(x, t) \in M \times\left(0, \frac{T_{2}}{2}\right] .
$$

Then

$$
\begin{aligned}
\left|\lim _{t \rightarrow 0}\left(g(x, t)-g_{0}(x)\right)\right| & =\left|\lim _{t \rightarrow 0} \lim _{k \rightarrow \infty}\left(g_{k}(x, t)-g_{k}(x, 0)\right)\right| \\
& =\left|\lim _{t \rightarrow 0} \lim _{k \rightarrow \infty} \int_{0}^{t} \frac{\partial}{\partial s} g_{k}(x, s) d s\right| \\
& \leq \lim _{t \rightarrow 0} \lim _{k \rightarrow \infty} \int_{0}^{t}\left|2 \chi_{k}^{2} R c_{k}\right|_{\infty} \\
& \leq C \lim _{t \rightarrow 0}\left(t^{1-\frac{n}{2 p_{0}}}+t^{1+\frac{1}{p_{0}}}\right)=0 .
\end{aligned}
$$

In the last inequality above, we used (5.21). Hence we can define

$$
g(x, 0)=g_{0}(x), \quad x \in M^{n} .
$$

Then by (7.2), (7.12) and (7.13), we get that $g(t)$ is the solution of the Ricci flow (1.1) on $M^{n} \times[0, T]$. This is the first conclusion of Theorem 1.1 there remains to prove the estimates (1.4).

Now by (7.5) and (7.12),

$$
\left|R m_{g}\left(x, t_{1}\right)\right|_{\infty} \leq \lim _{k \rightarrow \infty}\left|R m_{k}\left(x, t_{1}\right)\right|_{\infty} \leq C_{2}\left(t_{1}^{-\frac{n}{2 p_{0}}}+C_{1}^{\frac{n+2}{2 p_{0}}} t_{1}^{\frac{1}{p_{0}}}\right)
$$

for any $x \in M^{n}$ and $t_{1} \in\left(0, \frac{T_{2}}{2}\right]$.

We take $\left(M^{n}, g\left(\frac{t_{1}}{2}\right)\right)$ as the initial conditions of the Ricci flow. Then the Ricci flow has a solution on $M^{n} \times\left[\frac{t_{1}}{2}, \frac{T_{2}}{2}\right]$ and

$$
\left|R m_{g\left(\frac{t_{1}}{2}\right)}\right|_{\infty} \leq C\left(A_{0}, K_{1}, n, p_{0}, r_{0}\right)\left(t_{1}^{-\frac{n}{2 p_{0}}}+t_{1}^{\frac{1}{p_{0}}}\right) .
$$

Now define

$$
\tilde{g}(x, t)=\left[C\left(t_{1}^{-\frac{n}{2 p_{0}}}+t_{1}^{\frac{1}{p_{0}}}\right)\right] \cdot g\left(x, \frac{t_{1}}{2}+t\left[C\left(t_{1}^{-\frac{n}{2 p_{0}}}+t_{1}^{\frac{1}{p_{0}}}\right)\right]^{-1}\right),
$$

where $C=C\left(A_{0}, K_{1}, n, p_{0}, r_{0}\right)$ in (7.15) is the same as in (7.14). Then from the scaling argument,

$$
\left\{\begin{aligned}
\frac{\partial}{\partial t} \tilde{g}_{i j}(x, t) & =-2 \tilde{R}_{i j}(x, t), \\
\tilde{g}(x, 0) & =\left[C\left(A_{0}, K_{1}, n, p_{0}, r_{0}\right)\left(t_{1}^{-\frac{n}{2 p_{0}}}+t_{1}^{\frac{1}{p_{0}}}\right)\right] g\left(x, \frac{t_{1}}{2}\right) .
\end{aligned}\right.
$$

We already know that $g_{i j}$ and its Ricci flow exist on $\left[\frac{t_{1}}{2}, \frac{T_{2}}{2}\right]$ and that $\tilde{g}_{i j}$ and its Ricci flow exist on $\left[0,\left(\frac{T_{2}}{2}-\frac{t_{1}}{2}\right) C\left(A_{0}, K_{1}, n, p_{0}, r_{0}\right)\left(t_{1}^{-\frac{n}{2 p_{0}}}+t_{1}^{\frac{1}{p_{0}}}\right)\right]$. We use $\left\{\tilde{R}_{i j k l}(x)\right\}$ and $\tilde{\nabla}$ to denote, respectively, the Riemannian curvature tensor and the covariant derivative with respect to $\tilde{g}_{i j}(x, 0)$.

Note that $|\widetilde{R m}| \leq 1$ on $M$, and by Lemma 7.1 of [12, there exists a positive constant $C(n, m)$, such that for any $m \geq 0$, if

$$
t \in\left(0,\left(\frac{T_{2}}{2}-\frac{t_{1}}{2}\right) C\left(A_{0}, K_{1}, n, p_{0}, r_{0}\right)\left(t_{1}^{-\frac{n}{2 p_{0}}}+t_{1}^{\frac{1}{p_{0}}}\right)\right],
$$

we have

$$
\left|\tilde{\nabla}^{m} \widetilde{R m}(x, t)\right|^{2} \leq \frac{C(n, m)}{t^{m}}
$$


By (7.15) and (7.16), for $\frac{t_{1}}{2}<t \leq \frac{T_{2}}{2}$,

$$
\begin{aligned}
\left|\nabla^{m} R m(x, t)\right|^{2} & \leq \frac{C(n, m)\left[C\left(A_{0}, K_{1}, n, p_{0}, r_{0}\right)\left(t_{1}^{-\frac{n}{2 p_{0}}}+t_{1}^{\frac{1}{p_{0}}}\right)\right]^{m+2}}{\left[C\left(A_{0}, K_{1}, n, p_{0}, r_{0}\right)\left(t_{1}^{-\frac{n}{2 p_{0}}}+t_{1}^{\frac{1}{p_{0}}}\right)\left(t-\frac{t_{1}}{2}\right)\right]^{m}} \\
& \leq \frac{C\left(A_{0}, K_{1}, m, n, p_{0}, r_{0}\right)}{\left(t-t_{1}\right)^{m}}\left(t_{1}^{-\frac{n}{2 p_{0}}}+t_{1}^{\frac{1}{p_{0}}}\right)^{2} .
\end{aligned}
$$

Then

$$
\left|\nabla^{m} R m\left(x, t_{1}\right)\right|^{2} \leq \frac{C\left(A_{0}, K_{1}, m, n, p_{0}, r_{0}\right)}{t_{1}^{m}}\left(t_{1}^{-\frac{n}{2 p_{0}}}+t_{1}^{\frac{1}{p_{0}}}\right)^{2}, \quad m \geq 0 .
$$

Because $t_{1}$ was chosen arbitrarily,

$$
\left|\nabla^{m} R m(x, t)\right| \leq \frac{C\left(A_{0}, K_{1}, m, n, p_{0}, r_{0}\right)}{t^{\frac{m}{2}}}\left(t^{-\frac{n}{2 p_{0}}}+t^{\frac{1}{p_{0}}}\right), \text { for } 0<t \leq \frac{T_{2}}{2}, m \geq 0 .
$$

Remark 7.1. In fact, the assumption about the integral of the curvature tensor in the above theorem can be weakened as

$$
\left(f_{B_{x_{i}}\left(r_{0}\right)}|R m|^{p_{0}}\right)^{\frac{1}{p_{0}}} \leq K_{1}
$$

where $B_{x_{i}}\left(r_{0}\right)$ is chosen as in Lemma 5.4 ,

Remark 7.2. By Remark 5.15 and the argument in Theorem 1.1 if $\left(M^{n}, g_{0}\right)$ satisfies (5.29), the Ricci flow (1.1) has a smooth solution on $[0, T]$, where $T>0$ is some positive constant.

Proof of Corollary [1.2, Because $R c\left(g_{0}\right) \geq-K g_{0}$, by Theorem 3.1 in [10] and Corollary 1.1 in [8], $A_{0} \leq C\left(n, K, r_{0}\right)$. Then applying Theorem 1.1, we get our conclusion.

We also have the following corollary which is part of Shi's Theorem.

Corollary 7.3. Let $\left(M, g_{0}\right)$ be an $n$-dimensional complete noncompact Riemannian manifold with its Riemannian curvature tensor $\left\{R_{i j k l}\right\}$ satisfying $|R m| \leq k_{0}$ on $M$, where $0<k_{0}<+\infty$ is a constant. Then there is a constant $T\left(n, k_{0}\right)>0$ depending only on $n$ and $k_{0}$ such that the evolution equation (1.1) has a smooth solution $g_{i j}(x, t)$ on $\left[0, T\left(n, k_{0}\right)\right]$.

Proof. By $|R m| \leq k_{0}$, there exists $K=n k_{0}$ such that

$$
R c\left(g_{0}\right) \geq-K g_{0},
$$

and it is obvious that

$$
\left(f_{\tilde{B}_{x}(1)}\left|R m\left(g_{0}\right)\right|^{n} d V_{g_{0}}\right)^{\frac{1}{n}} \leq k_{0} .
$$

Now we choose

$$
K=n k_{0}, K_{1}=k_{0}, p_{0}=n, r=1 .
$$

Then by all the above choices of $K, K_{1}, p_{0}$ and $r$, (1.6) in Corollary 1.2 is satisfied. We get our conclusion.

By Theorem 6.10, we can use a similar argument in the proof of Theorem 1.1 to get the following theorem. 
Theorem 7.4. Assume $\left(M^{n}, g_{0}\right)$ is an $n$-dimensional $(n \geq 3)$ complete noncompact Riemannian manifold satisfying (6.1). Then the Ricci flow (1.1) has a smooth solution $g_{i j}(x, t)$ on $\left[0, \widehat{T}_{4}\right]$ (where $\widehat{T}_{4}$ is defined in (6.23) and satisfies the following estimates. For any integer $m \geq 0$, there exists a positive constant $C\left(\widehat{A}_{0}, \widehat{K}_{1}, \widehat{N}_{1}, m, n, r_{0}\right)$ such that

$$
\sup _{x \in M^{n}}\left|\nabla^{m} R m(x, t)\right| \leq \frac{C\left(\widehat{A}_{0}, \widehat{K}_{1}, \widehat{N}_{1}, m, n, r_{0}\right)}{t^{\frac{m}{2}}}\left(t^{-1}+t\right), \quad 0<t \leq \frac{\widehat{T}_{4}}{2} .
$$

Especially,

$$
\sup _{x \in M^{n}}|R m(x, t)| \leq C\left(n, \widehat{N}_{1}, r_{0}\right)\left(t^{-1}+t\right), \quad 0<t \leq \frac{\widehat{T}_{4}}{2},
$$

where $C\left(n, \widehat{N}_{1}, r_{0}\right)$ depends only on $n, \widehat{N}_{1}$ and $r_{0}$.

\section{ACKNOWLEDGMENT}

The author is grateful to his advisor, Professor Robert Gulliver, for reading this paper and providing comments and suggestions. He would like to thank his teacher, Professor Jiaping Wang, for suggesting this problem and bringing his attention to the article [15], also for his encouragement and many helpful discussions. He would like to thank King-Yeung Lam, Weiwei Wu and Weiyi Zhang for useful discussions.

\section{References}

[1] Bennett Chow and Dan Knopf, The Ricci flow: an introduction, Mathematical Surveys and Monographs, vol. 110, American Mathematical Society, Providence, RI, 2004. MR2061425 (2005e:53101)

[2] Bennett Chow, Sun-Chin Chu, David Glickenstein, Christine Guenther, James Isenberg, Tom Ivey, Dan Knopf, Peng Lu, Feng Luo, and Lei Ni, The Ricci flow: techniques and applications. Part I, Mathematical Surveys and Monographs, vol. 135, American Mathematical Society, Providence, RI, 2007. Geometric aspects. MR2302600(2008f:53088)

[3] Bennett Chow, Sun-Chin Chu, David Glickenstein, Christine Guenther, James Isenberg, Tom Ivey, Dan Knopf, Peng Lu, Feng Luo, and Lei Ni, The Ricci flow: techniques and applications. Part II, Mathematical Surveys and Monographs, vol. 144, American Mathematical Society, Providence, RI, 2008. Analytic aspects. MR2365237 (2008j:53114)

[4] Dennis M. DeTurck, Deforming metrics in the direction of their Ricci tensors, J. Differential Geom. 18 (1983), no. 1, 157-162. MR697987 (85j:53050)

[5] Richard S. Hamilton, Three-manifolds with positive Ricci curvature, J. Differential Geom. 17 (1982), no. 2, 255-306. MR664497 (84a:53050)

[6] Emmanuel Hebey, Nonlinear analysis on manifolds: Sobolev spaces and inequalities, Courant Lecture Notes in Mathematics, vol. 5, New York University Courant Institute of Mathematical Sciences, New York, 1999. MR1688256 (2000e:58011)

[7] Sergiu Klainerman, Global existence for nonlinear wave equations, Comm. Pure Appl. Math. 33 (1980), no. 1, 43-101, DOI 10.1002/cpa.3160330104. MR.544044 (81b:35050)

[8] Peter Li and Richard Schoen, $L^{p}$ and mean value properties of subharmonic functions on Riemannian manifolds, Acta Math. 153 (1984), no. 3-4, 279-301, DOI 10.1007/BF02392380. $\operatorname{MR} 766266(86 \mathrm{j}: 58147)$

[9] Gary M. Lieberman, Second order parabolic differential equations, World Scientific Publishing Co. Inc., River Edge, NJ, 1996. MR1465184 (98k:35003)

[10] Laurent Saloff-Coste, Uniformly elliptic operators on Riemannian manifolds, J. Differential Geom. 36 (1992), no. 2, 417-450. MR.1180389 (93m:58122)

[11] Laurent Saloff-Coste, Aspects of Sobolev-type inequalities, London Mathematical Society Lecture Note Series, vol. 289, Cambridge University Press, Cambridge, 2002. MR1872526 (2003c:46048) 
[12] Wan-Xiong Shi, Deforming the metric on complete Riemannian manifolds, J. Differential Geom. 30 (1989), no. 1, 223-301. MR.1001277 (90i:58202)

[13] Wan-Xiong Shi, Ricci flow and the uniformization on complete noncompact Kähler manifolds, J. Differential Geom. 45 (1997), no. 1, 94-220. MR1443333(98d:53099)

[14] Yuanqi Wang, Pseudolocality of Ricci Flow under Integral Bound of Curvature, to appear in J. Geometric Analysis.

[15] Deane Yang, Convergence of Riemannian manifolds with integral bounds on curvature. I, Ann. Sci. École Norm. Sup. (4) 25 (1992), no. 1, 77-105. MR1152614 (93a:53037)

Department of Mathematics, University of California, Irvine, California 92617

E-mail address: guoyixu@math.uci.edu

Current address: Mathematical Sciences Center, Tsinghua University, Beijing, 100084, People's Republic of China

E-mail address: guoyi.xu@gmail.com 